Supporting information

\title{
Experimental and Computational Studies of Phosphine Ligand Displacement in Iridium Pincer Complexes Employing Pyridine or Acetonitrile
}

Sara Shafiei-Haghighi ${ }^{\dagger}$, Aneelman Brar ${ }^{\dagger}$, Daniel K. Unruh, Anthony F. Cozzolino* and Michael Findlater*

Department of Chemistry and Biochemistry, Texas Tech University, Lubbock, TX 79409, USA

\section{Contents}

1. General Information: 2

2. Spectral data for displacement of $\mathrm{PPh}_{3}$ by pyridine 3

3. Spectral data for displacement of $\mathrm{PPh}_{3}$ by acetonitrile 3

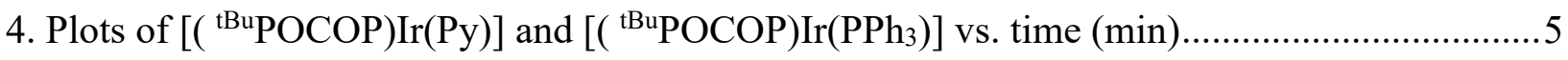

5. Plots of $\left[\left({ }^{\mathrm{tBu}} \mathrm{POCOP}\right) \operatorname{Ir}\left(\mathrm{NCCH}_{3}\right)\right]$ and $\left[\left({ }^{\mathrm{tBu}} \mathrm{POCOP}\right) \operatorname{Ir}\left(\mathrm{PPh}_{3}\right)\right]$ vs. time $(\mathrm{min})$...................... 7

6. Table S1. Equilibrium constant and equilibrium $\Delta \mathrm{G}$ at $348.15 \mathrm{~K}$ determined by ${ }^{1} \mathrm{H}$ NMR in toluene- $d 8$.

7. Comparison of experimental and DFT calculations in terms of bond parameters and energy...9

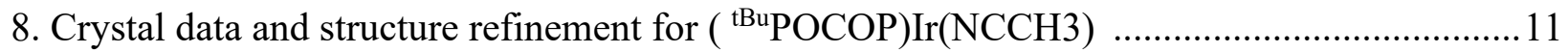

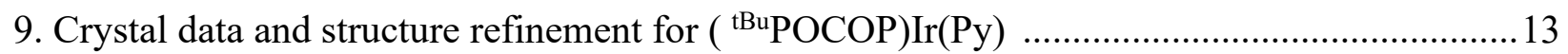

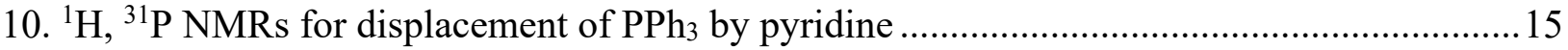

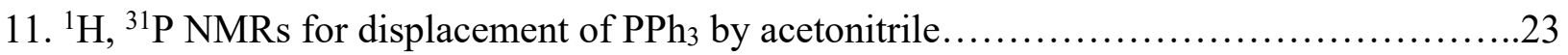

12. ${ }^{1} \mathrm{H},{ }^{31} \mathrm{P}$ NMRs for addition of free phosphine to reaction of $\mathrm{Ir}-\mathrm{PPh}_{3}$ and pyridine ...............31

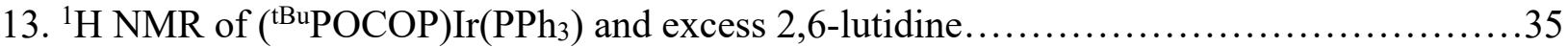

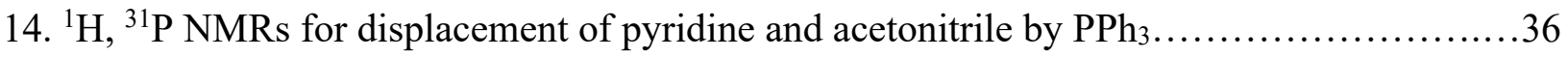

15.FTIR of ( $\left.{ }^{\mathrm{tBu}} \mathrm{POCOP}\right) \operatorname{Ir}\left(\mathrm{PPh}_{3}\right),\left({ }^{\mathrm{tBu}} \mathrm{POCOP}\right) \operatorname{Ir}(\mathrm{Py}) \&\left({ }^{\mathrm{tBu}} \mathrm{POCOP}\right) \operatorname{Ir}\left(\mathrm{NCCH}_{3}\right) \ldots \ldots \ldots \ldots \ldots . . . . .41$

16. UV-vis Kinetic Data and Calculations..............................................44 


\section{General Considerations}

All reactions were performed in oven dried apparatus under the atmosphere of Argon. All reagents were purchased from commercial vendors and were used as received without any purification or drying. ${ }^{1} \mathrm{H},{ }^{13} \mathrm{C}$, and ${ }^{31} \mathrm{P}$ were recorded on a Jeol $400 \mathrm{MHz}$ spectrometer. ${ }^{1} \mathrm{H}$ NMR spectra were referenced to the solvent residual peak (toluene- $d_{8}, \delta 2.09 \mathrm{ppm}$ ). Data for ( $\left.{ }^{\mathrm{BBu}} \mathrm{POCOP}\right) \operatorname{Ir}\left(\mathrm{NCCH}_{3}\right)$ complex were collected on a Rigaku XtaLAB Synergy- $i$ Kappa diffractometer equipped with a PhotonJet- $i \mathrm{X}$-ray source operated at $50 \mathrm{~W}(50 \mathrm{kV}, 1 \mathrm{~mA})$ to generate $\mathrm{Cu} \mathrm{K} \alpha$ radiation $(\lambda=1.54178$ $\AA)$ and a HyPix-6000HE HPC detector. Crystals were transferred from the vial and placed on a glass slide in polyisobutylene. A Zeiss Stemi 305 microscope was used to identify a suitable specimen for X-ray diffraction from a representative sample of the material. The crystal and a small amount of the oil were collected on a MīTiGen cryoloop and transferred to the instrument where it was placed under a cold nitrogen stream (Oxford 700 series) maintained at $100 \mathrm{~K}$ throughout the duration of the experiment. The sample was optically centered with the aid of a video camera to ensure that no translations were observed as the crystal was rotated through all positions. 
2. Spectral data for Displacement of $\mathrm{PPh}_{3}$ by pyridine

Equilibrium between ( $\left.{ }^{\mathrm{tBu}} \mathrm{POCOP}\right) \operatorname{Ir}\left(\mathrm{PPh}_{3}\right)$ and $\left({ }^{\mathrm{tBu}} \mathrm{POCOP}\right) \operatorname{Ir}(\mathrm{Py})$ using 2 equivalents of Pyridine: $\delta_{\mathrm{H}}\left(400 \mathrm{MHz}\right.$; toluene- $\left.d_{8}\right): \delta 9.10$ (d, $\left.J=5.2 \mathrm{~Hz}, 1 \mathrm{H}, \mathrm{Ir}-\mathrm{py}\right), 8.45$ (brs, 4H, py), 8.02 (t, $\left.J=8.0 \mathrm{~Hz}, 3 \mathrm{H}, \mathrm{Ir}-\mathrm{PPh}_{3}\right), 7.32\left(\mathrm{~m}, 3 \mathrm{H}, \mathrm{PPh}_{3}\right), 7.12\left(\mathrm{~m}, 1 \mathrm{H}, \mathrm{Ir}-\mathrm{PPh}_{3}\right), 7.03-7.00$ (m, 9H, Ir-PPh$\underline{3}$, $\mathrm{PPh}_{3}$, Ir-py, py), 6.83 (t, J=8.0 Hz, 1H, Ir-PPh $), 6.73-6.70$ (m, 4H, py), 6.67-6.65 (m, 1H, Ir-py), 6.43 (t, $J=6.4 \mathrm{~Hz}, 1 \mathrm{H}, \mathrm{Ir}-\mathrm{py}), 1.29$ (t, $J=6.4 \mathrm{~Hz}, 18 \mathrm{H}, \mathrm{Ir}-\mathrm{py}), 1.04$ (t, $\left.J=6.4 \mathrm{~Hz}, 18 \mathrm{H}, \mathrm{Ir}-\mathrm{PPh}_{3}\right) .{ }^{31} \mathrm{P}$ $\left\{{ }^{1} \mathrm{H}\right\}\left(162 \mathrm{MHz}\right.$, toluene- $\left.d_{8}\right): \delta 180.6\left(\mathrm{~d}, J=6.5 \mathrm{~Hz}, 2 \mathrm{P}, \mathrm{Ir}-\mathrm{PPh}_{3}\right), 172.6(\mathrm{~s}, 2 \mathrm{P}, \mathrm{Ir}-\mathrm{py}), 15.64(\mathrm{t}, J=$ $\left.5.8 \mathrm{~Hz}, 1 \mathrm{P}, \mathrm{Ir}_{-} \mathrm{PPh}_{3}\right), 4.69\left(\mathrm{~s}, 1 \mathrm{P}, \mathrm{PPh}_{3}\right)$.

Equilibrium between ( $\left.{ }^{\mathrm{tBu}} \mathrm{POCOP}\right) \operatorname{Ir}\left(\mathrm{PPh}_{3}\right)$ and $\left({ }^{\mathrm{tBu}} \mathrm{POCOP}\right) \operatorname{Ir}(\mathrm{Py})$ using 4 equivalents of Pyridine: $\delta_{\mathrm{H}}\left(400 \mathrm{MHz}\right.$; toluene- $\left.d_{8}\right): \delta 9.06$ (d, $\left.J=4.8 \mathrm{~Hz}, 1 \mathrm{H}, \mathrm{Ir}-\mathrm{py}\right), 8.49$ (d, $\left.J=4.0 \mathrm{~Hz}, 8 \mathrm{H}, \mathrm{py}\right)$, 8.06 (t, $\left.J=8.4 \mathrm{~Hz}, 2 \mathrm{H}, \mathrm{Ir}-\mathrm{PPh}_{3}\right), 7.34-7.30\left(\mathrm{~m}, 4 \mathrm{H}, \mathrm{PPh}_{3}\right), 7.10\left(\mathrm{~s}, 1 \mathrm{H}, \mathrm{Ir}_{-} \mathrm{PPh}_{3}\right), 7.07-7.00(\mathrm{~m}, 11 \mathrm{H}$, $\left.\mathrm{Ir}^{-\mathrm{PPh}_{3}}, \mathrm{PPh}_{3}, \mathrm{Ir}-\mathrm{py}, \mathrm{py}\right), 6.94-6.90$ (m, 1H, Ir-py), 6.82 (dd, $J=7.6 \mathrm{~Hz}, 1 \mathrm{H}, \mathrm{Ir}_{\mathrm{P}} \mathrm{PPh}_{3}$ ), 6.79-6.77 (m, 1H, Ir-py), 6.69-6.66 (m, 8H, py), 6.33 (dq, $J=6.4$ Hz, 1H, Ir-py), 1.30 (t, $J=6.4$ Hz, 24H, Irpy), 1.04 (t, $\left.J=6.4 \mathrm{~Hz}, 12 \mathrm{H}, \mathrm{Ir}-\mathrm{PPh}_{3}\right) .{ }^{31} \mathrm{P}\left\{{ }^{1} \mathrm{H}\right\}\left(162 \mathrm{MHz}\right.$, toluene- $\left.d_{8}\right): \delta 180.6(\mathrm{~d}, J=6.5 \mathrm{~Hz}, 2 \mathrm{P}$, $\mathrm{Ir}_{\mathrm{PPh}}$ ), 172.6 (s, 1P, Ir-py), 15.64 (t, J=5.7 Hz, 1P, Ir-PPh $), 4.69$ (s, 1P, $\left.\mathrm{PPh}_{3}\right)$.

Equilibrium between ( $\left.{ }^{\mathrm{tBu}} \mathrm{POCOP}\right) \operatorname{Ir}\left(\mathrm{PPh}_{3}\right)$ and $\left({ }^{\mathrm{tBu}} \mathrm{POCOP}\right) \operatorname{Ir}(\mathrm{Py})$ using 8 equivalents of Pyridine: $\delta_{\mathrm{H}}\left(400 \mathrm{MHz}\right.$; toluene- $\left.d_{8}\right): \delta 9.04(\mathrm{~d}, J=4.8 \mathrm{~Hz}, 1 \mathrm{H}, \mathrm{Ir}-\mathrm{py}), 8.48$ (d, $\left.J=4.0 \mathrm{~Hz}, 20 \mathrm{H}, \mathrm{py}\right)$, 8.04 (t, $\left.J=9.2 \mathrm{~Hz}, 1 \mathrm{H}, \mathrm{Ir}-\underline{\mathrm{PPh}}_{3}\right), 7.34-7.30$ (m, 4H, PPh $), 7.13\left(\mathrm{~s}, 1 \mathrm{H}, \mathrm{Ir}_{2} \mathrm{PPh}_{3}\right), 7.07-7.02(\mathrm{~m}, 15 \mathrm{H}$, $\left.\mathrm{Ir}^{-\mathrm{PPh}_{3}}, \mathrm{PPh}_{3}, \mathrm{Ir}-\mathrm{py}, \mathrm{py}\right), 6.94-6.90$ (m, 1H, Ir-py), 6.82 (dd, $\left.J=8.0 \mathrm{~Hz}, 1 \mathrm{H}, \mathrm{Ir}_{\mathrm{P}} \mathrm{PPh}_{3}\right), 6.78-6.77$ (m, 1H, Ir-py), 6.69-6.66 (m, 20H, py), 6.34 (dq, $J=6.2 \mathrm{~Hz}, 1 \mathrm{H}, \mathrm{Ir}-\mathrm{py}), 1.30$ (t, $J=6.4 \mathrm{~Hz}, 27 \mathrm{H}$, Ir-py), $1.04(\mathrm{t}, J=6.4 \mathrm{~Hz}, 9 \mathrm{H}, \mathrm{Ir}-\mathrm{PPh}) .{ }^{31} \mathrm{P}\left\{{ }^{1} \mathrm{H}\right\}\left(162 \mathrm{MHz}\right.$, toluene- $\left.d_{8}\right): \delta 180.6(\mathrm{~d}, J=5.7 \mathrm{~Hz}, 1 \mathrm{P}$, Ir-PPh $), 172.6$ (s, 4P, Ir-py), 15.62 (t, J=5.5 Hz, 1P, Ir-PPh $), 4.69$ (s, 2P, $\mathrm{PPh}_{3}$ ).

Equilibrium between ( $\left.{ }^{\mathrm{tBu} P O C O P}\right) \operatorname{Ir}\left(\mathrm{PPh}_{3}\right)$ and ( $\left.{ }^{\mathrm{tBu} P O C O P}\right) \operatorname{Ir}(\mathrm{Py})$ using 16 equivalents of Pyridine: $\delta_{\mathrm{H}}\left(400 \mathrm{MHz}\right.$; toluene- $\left.d_{8}\right): \delta 9.04(\mathrm{~d}, J=4.8 \mathrm{~Hz}, 1 \mathrm{H}, \mathrm{Ir}-\mathrm{py}), 8.48$ (d, $J=4.0 \mathrm{~Hz}, 32 \mathrm{H}$, py), 8.04 (t, $\left.J=8.0 \mathrm{~Hz}, 1 \mathrm{H}, \mathrm{Ir}-\underline{\mathrm{PPh}}_{3}\right), 7.34-7.30\left(\mathrm{~m}, 5 \mathrm{H}, \mathrm{PPh}_{3}\right), 7.13\left(\mathrm{~s}, 1 \mathrm{H}, \mathrm{Ir}_{-} \mathrm{PPh}_{3}\right), 7.07-7.00(\mathrm{~m}, 21 \mathrm{H}$, $\left.\mathrm{Ir}-\underline{\mathrm{PPh}}_{3}, \mathrm{PPh}_{3}, \mathrm{Ir}-\mathrm{py}, \mathrm{py}\right), 6.93-6.89$ (m, 1H, Ir-py), 6.82 (dd, $J=7.6 \mathrm{~Hz}, 1 \mathrm{H}, \mathrm{Ir}_{\mathrm{H}} \mathrm{PPh}_{3}$ ), 6.78-6.76 (m, 1H, Ir-py), 6.68 (dq, $J=7.8 \mathrm{~Hz}, 32 \mathrm{H}$, py), 6.35 (dq, $J=6.4 \mathrm{~Hz}, 1 \mathrm{H}, \mathrm{Ir}-\mathrm{py}), 1.30$ (t, $J=6.4 \mathrm{~Hz}$, $32 \mathrm{H}, \mathrm{Ir}-\mathrm{py}), 1.04$ (t, $\left.J=6.4 \mathrm{~Hz}, 4 \mathrm{H}, \mathrm{Ir}-\mathrm{PPh}_{3}\right) .{ }^{31} \mathrm{P}\left\{{ }^{1} \mathrm{H}\right\}\left(162 \mathrm{MHz}\right.$, toluene- $\left.d_{8}\right): \delta 180.6(\mathrm{~d}, J=6.0$ $\mathrm{Hz}, 1 \mathrm{P}, \mathrm{Ir}-\mathrm{PPh}_{3}$ ), 172.6 (s, 8P, Ir-py), 15.60 (t, J=5.8 Hz, 1P, Ir-PPh $), 4.69$ (s, 4P, $\left.\mathrm{PPh}_{3}\right)$.

\section{Spectral data for Displacement of $\mathrm{PPh}_{3}$ by acetonitrile}

Equilibrium between ( $\left.{ }^{\mathrm{tBu}} \mathrm{POCOP}\right) \operatorname{Ir}\left(\mathrm{PPh}_{3}\right)$ and $\left({ }^{\mathrm{tBu}} \mathrm{POCOP}\right) \operatorname{Ir}\left(\mathrm{NCCH}_{3}\right)$ using 2 equivalents of acetonitrile: $\delta_{\mathrm{H}}\left(400 \mathrm{MHz}\right.$; toluene- $\left.d_{8}\right): \delta 8.04\left(\mathrm{t}, J=9.2 \mathrm{~Hz}, 3 \mathrm{H}, \mathrm{Ir}-\underline{\mathrm{PPh}}_{3}\right), 7.34-7.30(\mathrm{~m}, 3 \mathrm{H}$, $\left.\mathrm{PPh}_{3}\right), 7.13$ (s, 1H, Ir-PPh $), 7.08-7.01$ (m, 9H, Ir- $\left.\mathrm{PPh}_{3}, \mathrm{PPh}_{3}\right), 6.88-6.84$ (m, 1H, Ir-NCCH $), 6.82$ (dd, $\left.J=7.6 \mathrm{~Hz}, 1 \mathrm{H}, \mathrm{Ir}-\mathrm{PPh}_{3}\right), 6.77-6.75\left(\mathrm{~m}, 1 \mathrm{H}, \mathrm{Ir}-\mathrm{NCCH}_{3}\right), 1.45\left(\mathrm{t}, J=6.8 \mathrm{~Hz}, 18 \mathrm{H}, \mathrm{Ir}-\mathrm{NCCH}_{3}\right)$, $1.04\left(\mathrm{t}, J=6.8 \mathrm{~Hz}, 18 \mathrm{H}, \mathrm{Ir}-\mathrm{PPh}_{3}\right), 0.68\left(\mathrm{~s}, 7 \mathrm{H}, \mathrm{NCCH}_{3}\right) .{ }^{31} \mathrm{P}\left\{{ }^{1} \mathrm{H}\right\}\left(162 \mathrm{MHz}\right.$, toluene- $\left.d_{8}\right): \delta 180.6$ (d, $\left.J=6.0 \mathrm{~Hz}, 2 \mathrm{P}, \mathrm{Ir}-\mathrm{PPh}_{3}\right), 179.5$ (s, 2P, Ir-NCCH 3$), 15.62$ (t, $\left.J=5.7 \mathrm{~Hz}, 1 \mathrm{P}, \mathrm{Ir}_{-} \mathrm{PPh}_{3}\right), 4.68(\mathrm{~s}, 1 \mathrm{P}$, $\mathrm{PPh}_{3}$ ). 
Equilibrium between ( $\left.{ }^{\mathrm{tBu}} \mathrm{POCOP}\right) \operatorname{Ir}\left(\mathrm{PPh}_{3}\right)$ and $\left({ }^{\mathrm{tBu}} \mathrm{POCOP}\right) \operatorname{Ir}\left(\mathrm{NCCH}_{3}\right)$ using 4 equivalents of acetonitrile: $\delta_{\mathrm{H}}\left(400 \mathrm{MHz}\right.$; toluene- $\left.d_{8}\right): \delta 8.06-8.01\left(\mathrm{~m}, 2 \mathrm{H}, \mathrm{Ir}-\mathrm{PPh}_{3}\right), 7.34-7.30\left(\mathrm{~m}, 4 \mathrm{H}, \mathrm{PPh}_{3}\right)$, $7.13\left(\mathrm{~s}, 1 \mathrm{H}, \mathrm{Ir}-\mathrm{PPh}_{3}\right), 7.09-7.03$ (m, 9H, Ir-PPh $\left.\underline{3}, \mathrm{PPh}_{3}\right), 6.88-6.84$ (m, 1H, Ir-NCCH 3$), 6.81$ (dd, $\left.J=7.8 \mathrm{~Hz}, 1 \mathrm{H}, \mathrm{Ir}-\mathrm{PPh}_{3}\right), 6.76-6.74\left(\mathrm{~m}, 1 \mathrm{H}, \mathrm{Ir}-\mathrm{NCCH}_{3}\right), 1.44$ (t, $\left.J=6.8 \mathrm{~Hz}, 24 \mathrm{H}, \mathrm{Ir}-\mathrm{NCCH}_{3}\right), 1.04$ (t, $\left.J=6.4 \mathrm{~Hz}, 12 \mathrm{H}, \mathrm{Ir}_{-} \mathrm{PPh}_{3}\right), 0.69\left(\mathrm{~s}, 16 \mathrm{H}, \mathrm{NCCH}_{3}\right) .{ }^{31} \mathrm{P}\left\{{ }^{1} \mathrm{H}\right\}\left(162 \mathrm{MHz}\right.$, toluene- $\left.d_{8}\right): \delta 180.5(\mathrm{~d}$, $\left.J=5.7 \mathrm{~Hz}, 1 \mathrm{P}, \mathrm{Ir}-\mathrm{PPh}_{3}\right), 179.4$ (s, 2P, Ir-NCCH 3$), 15.48$ (t, $\left.J=5.8 \mathrm{~Hz}, 1 \mathrm{P}, \mathrm{Ir}-\mathrm{PPh}_{3}\right), 4.8$ (s, 1P, $\left.\mathrm{PPh}_{3}\right)$.

Equilibrium between ( $\left.{ }^{\mathrm{tBu}} \mathrm{POCOP}\right) \operatorname{Ir}\left(\mathrm{PPh}_{3}\right)$ and $\left({ }^{\mathrm{tBu}} \mathrm{POCOP}\right) \operatorname{Ir}\left(\mathrm{NCCH}_{3}\right)$ using 8 equivalents of acetonitrile: $\delta_{\mathrm{H}}\left(400 \mathrm{MHz}\right.$; toluene- $\left.d_{8}\right): \delta 8.03\left(\mathrm{t}, J=9.4 \mathrm{~Hz}, 1 \mathrm{H}, \mathrm{Ir}-\underline{P P h}_{3}\right), 7.34-7.30(\mathrm{~m}, 4 \mathrm{H}$, $\left.\mathrm{PPh}_{3}\right), 7.13$ (s, 1H, Ir-PPh $)$, 7.07-7.02 (m, 9H, Ir- $\left.\mathrm{PPh}_{3}, \mathrm{PPh}_{3}\right), 6.87-6.83$ (m, 1H, Ir-NCCH $), 6.80$ $\left(\mathrm{dd}, J=8.0 \mathrm{~Hz}, 1 \mathrm{H}, \mathrm{Ir}-\mathrm{PPh}_{3}\right), 6.76-6.74\left(\mathrm{~m}, 1 \mathrm{H}, \mathrm{Ir}-\mathrm{NCCH}_{3}\right), 1.44$ (t, $J=6.8 \mathrm{~Hz}, 27 \mathrm{H}, \mathrm{Ir}-\mathrm{NCCH}_{3}$ ), $1.04\left(\mathrm{t}, J=6.4 \mathrm{~Hz}, 9 \mathrm{H}, \mathrm{Ir}_{-} \mathrm{PPh}_{3}\right), 0.71\left(\mathrm{~s}, 30 \mathrm{H}, \mathrm{NCCH}_{3}\right) .{ }^{31} \mathrm{P}\left\{{ }^{1} \mathrm{H}\right\}\left(162 \mathrm{MHz}\right.$, toluene- $\left.d_{8}\right): \delta 180.63$ (d, $\left.J=6.0 \mathrm{~Hz}, 1 \mathrm{P}, \mathrm{Ir}_{-} \mathrm{PPh}_{3}\right), 179.54$ (s, 4P, Ir-NCCH 3$), 15.55$ (t, J=5.7 Hz, 1P, Ir-PPh $), 4.71(\mathrm{~s}$, $\left.2 \mathrm{P}, \mathrm{PPh}_{3}\right)$.

Equilibrium between ( $\left.{ }^{\mathrm{tBu}} \mathrm{POCOP}\right) \operatorname{Ir}\left(\mathrm{PPh}_{3}\right)$ and $\left({ }^{\mathrm{tBu}} \mathrm{POCOP}\right) \operatorname{Ir}\left(\mathrm{NCCH}_{3}\right)$ using 16 equivalents of acetonitrile: $\delta_{\mathrm{H}}\left(400 \mathrm{MHz}\right.$; toluene- $\left.d_{8}\right): \delta 8.0\left(\mathrm{t}, J=8.2 \mathrm{~Hz}, 1 \mathrm{H}, \mathrm{Ir}-\underline{\mathrm{PPh}}_{3}\right), 7.31-7.27(\mathrm{~m}, 4 \mathrm{H}$, $\left.\mathrm{PPh}_{3}\right), 7.11$ (s, 1H, Ir-PPh $), 7.05-7.0$ (m, 9H, Ir- $\left.\underline{\mathrm{PPh}}_{3}, \mathrm{PPh}_{3}\right), 6.83-6.80$ (m, 1H, Ir-NCCH 3$), 6.76$ $\left(\mathrm{dd}, J=8.0 \mathrm{~Hz}, 1 \mathrm{H}, \mathrm{Ir}-\mathrm{PPh}_{3}\right), 6.71-6.69\left(\mathrm{~m}, 1 \mathrm{H}, \mathrm{Ir}-\mathrm{NCCH}_{3}\right), 1.41\left(\mathrm{t}, J=6.8 \mathrm{~Hz}, 32 \mathrm{H}, \mathrm{Ir}-\mathrm{NCCH}_{3}\right)$, $1.01\left(\mathrm{t}, J=6.0 \mathrm{~Hz}, 4 \mathrm{H}, \mathrm{Ir}_{-} \mathrm{PPh}_{3}\right), 0.73\left(\mathrm{~s}, 52 \mathrm{H}, \mathrm{NCCH}_{3}\right) .{ }^{31} \mathrm{P}\left\{{ }^{1} \mathrm{H}\right\}\left(162 \mathrm{MHz}\right.$, toluene- $\left.d_{8}\right): \delta 180.54$ (d, $\left.J=6.1 \mathrm{~Hz}, 1 \mathrm{P}, \mathrm{Ir}_{-} \mathrm{PPh}_{3}\right), 179.47$ (s, 8P, Ir-NCCH 3$), 15.44$ (t, $\left.J=5.7 \mathrm{~Hz}, 1 \mathrm{P}, \mathrm{Ir}_{3} \mathrm{PPh}_{3}\right), 4.81(\mathrm{~s}$, $\left.4 \mathrm{P}, \mathrm{PPh}_{3}\right)$. 


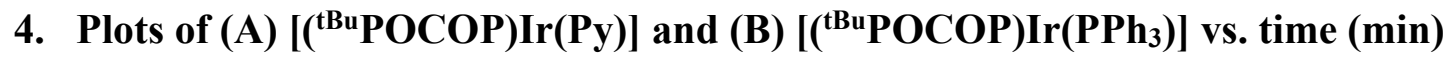

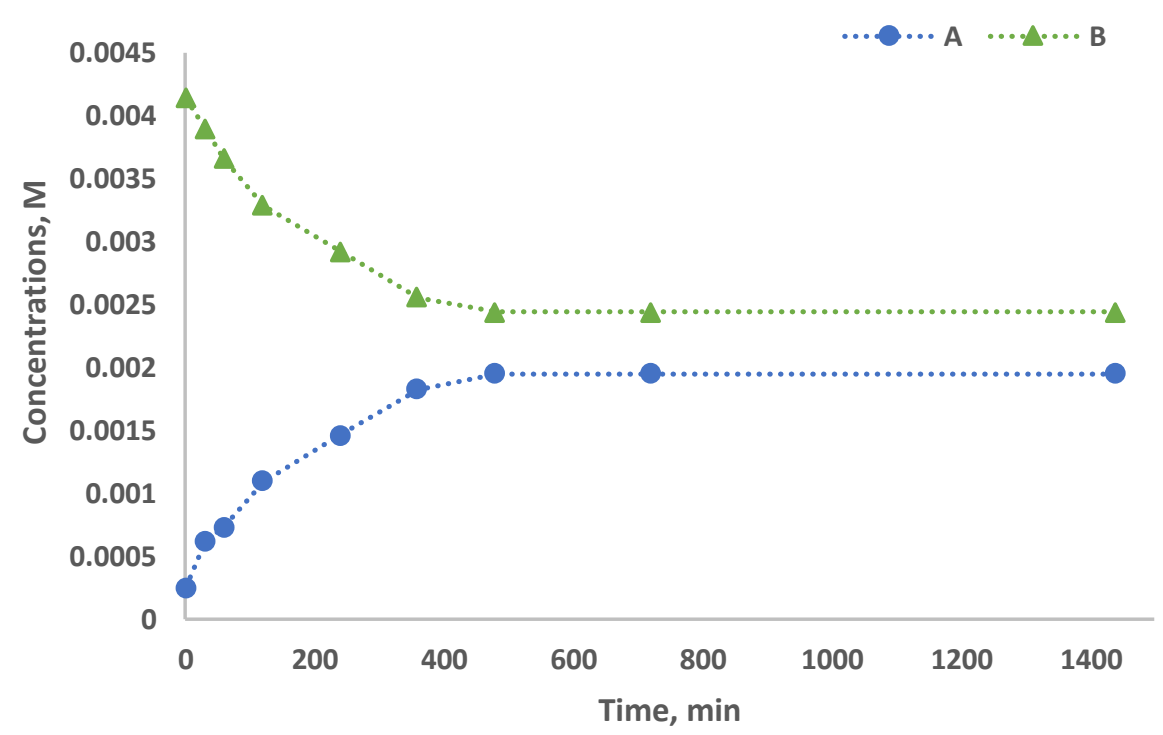

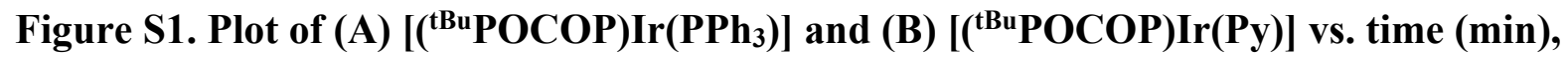
using 2 equivalents of Pyridine. The dotted lines added as a visual aid.

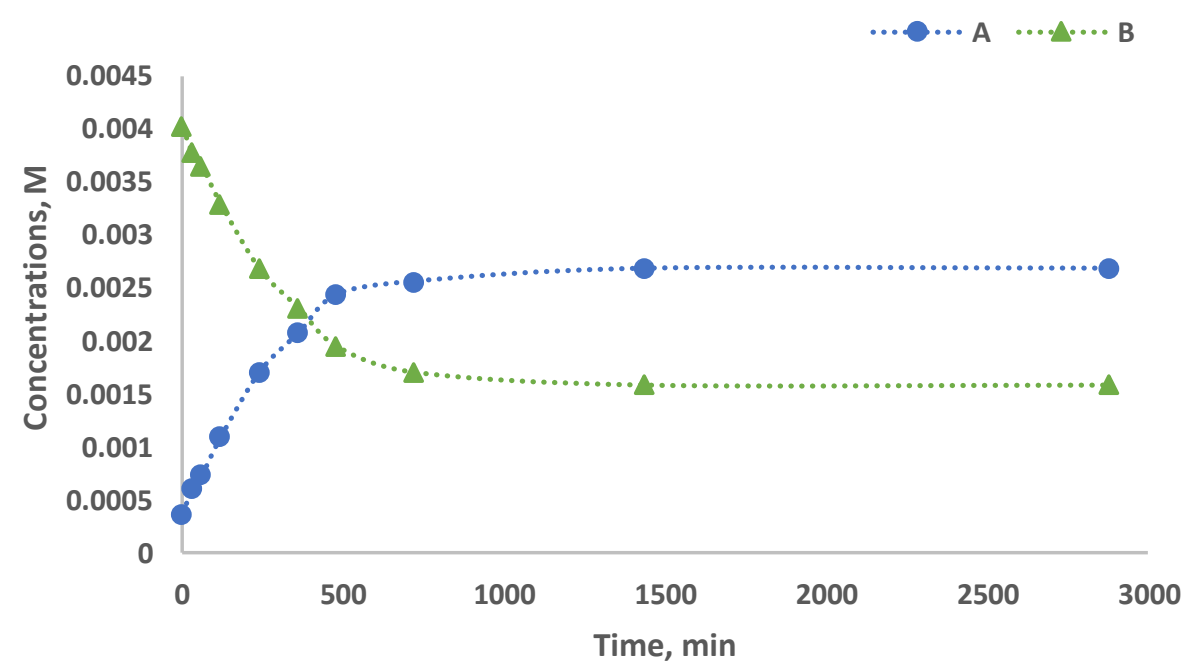

Figure S2. Plot of (A) [( $\left.\left.{ }^{\mathrm{tBu}} \mathrm{POCOP}\right) \operatorname{Ir}(\mathrm{Py})\right]$ and (B) $\left[\left({ }^{\mathrm{Bu}} \mathrm{POCOP}\right) \operatorname{Ir}\left(\mathrm{PPh}_{3}\right)\right]$ vs. time (min), using 4 equivalents of pyridine. The dotted lines added as a visual aid. 


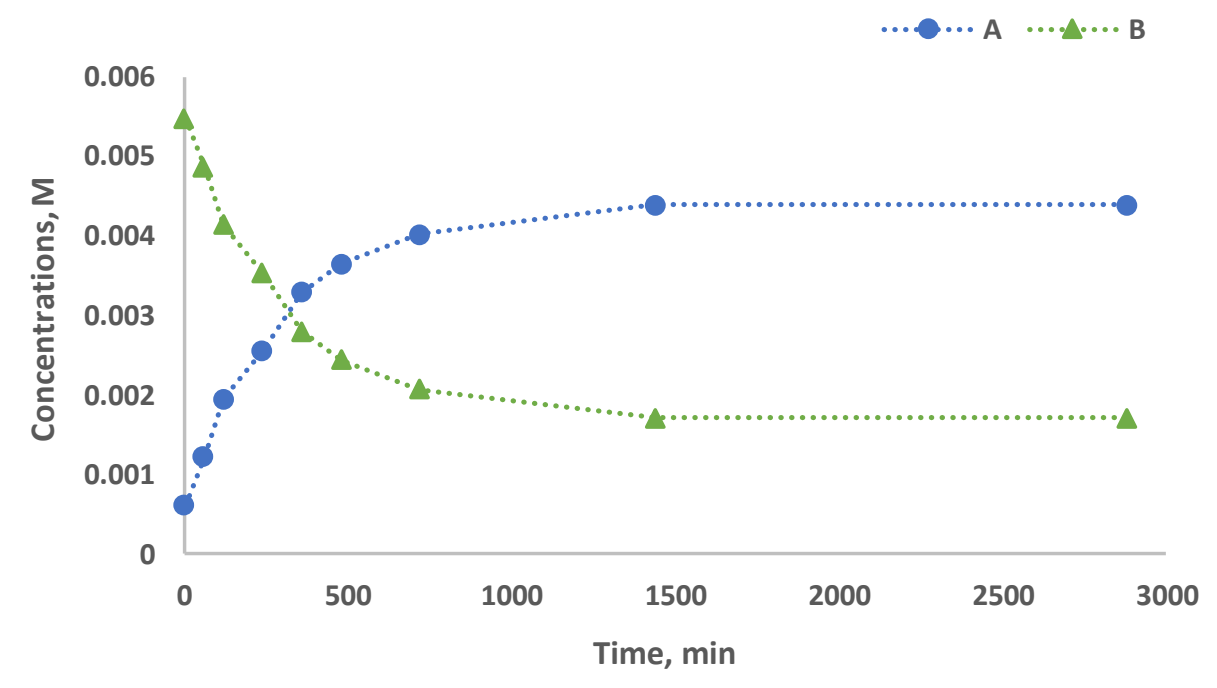

Figure S3. Plot of (A) [( $\left.\left.{ }^{\mathrm{tBu}} \mathrm{POCOP}\right) \operatorname{Ir}(\mathrm{Py})\right]$ and (B) $\left.\left[{ }^{\mathrm{tBu}} \mathrm{POCOP}\right) \operatorname{Ir}\left(\mathrm{PPh}_{3}\right)\right]$ vs. time (min), using 8 equivalents of pyridine. The dotted lines added as a visual aid.

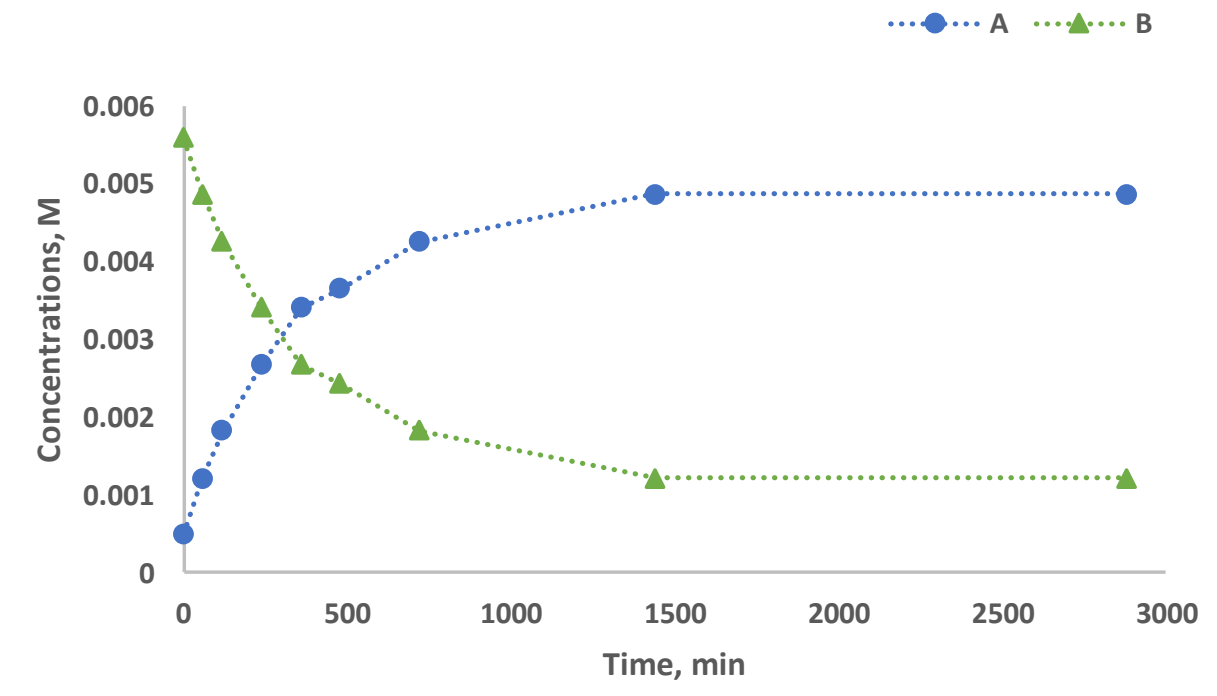

Figure S4. Plot of (A) [( $\left.\left.{ }^{\mathrm{tBu}} \mathrm{POCOP}\right) \operatorname{Ir}(\mathrm{Py})\right]$ and (B) $\left[\left({ }^{\mathrm{tBu}} \mathrm{POCOP}\right) \operatorname{Ir}\left(\mathrm{PPh}_{3}\right)\right]$ vs. time (min), using 16 equivalents of pyridine. The dotted lines added as a visual aid. 
5. Plots of (A) [ ( $\left.{ }^{\mathrm{tBu} P O C O P)} \operatorname{Ir}\left(\mathrm{NCCH}_{3}\right)\right]$ and $(\mathrm{B})\left[\left({ }^{\mathrm{tBu}} \mathrm{POCOP}\right) \operatorname{Ir}\left(\mathrm{PPh}_{3}\right)\right]$ vs. time (min)

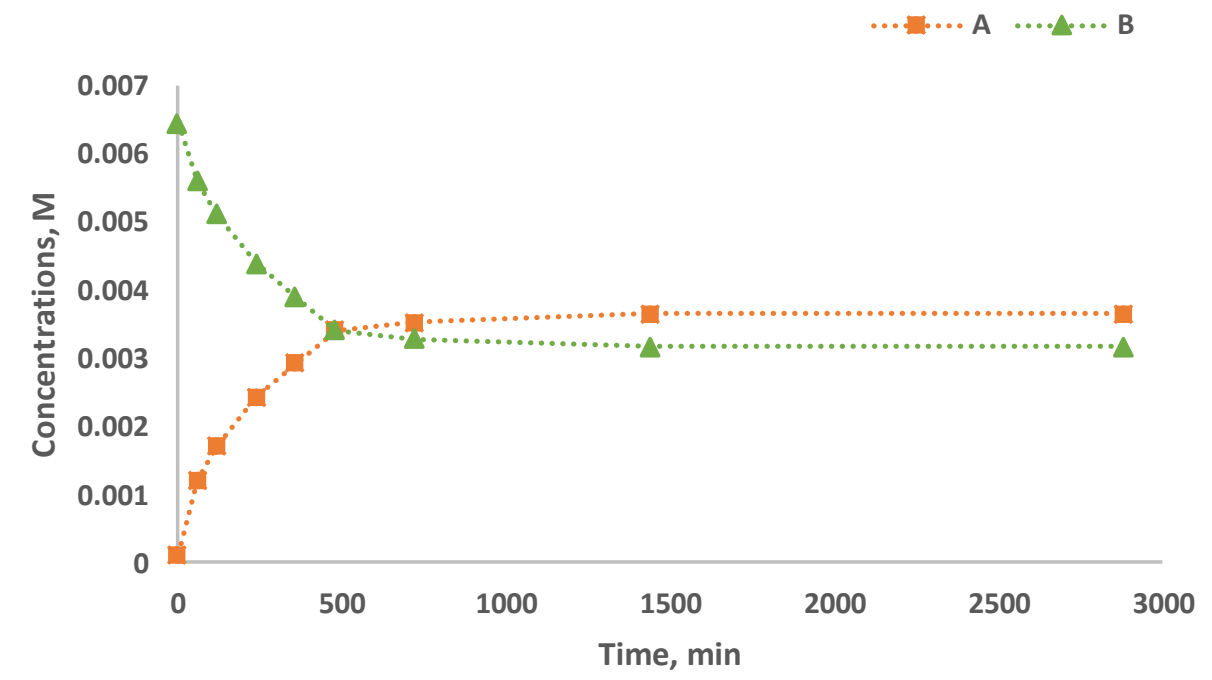

Figure S5. Plot of (A) [(tBuPOCOP) $\operatorname{Ir}(\mathrm{NCCH} 3)]$ and (B) $[(\mathrm{tBuPOCOP}) \operatorname{Ir}(\mathrm{PPh} 3)]$ vs. time (min), using 2 equivalents of $\mathrm{MeCN}$. The dotted lines added as a visual aid.

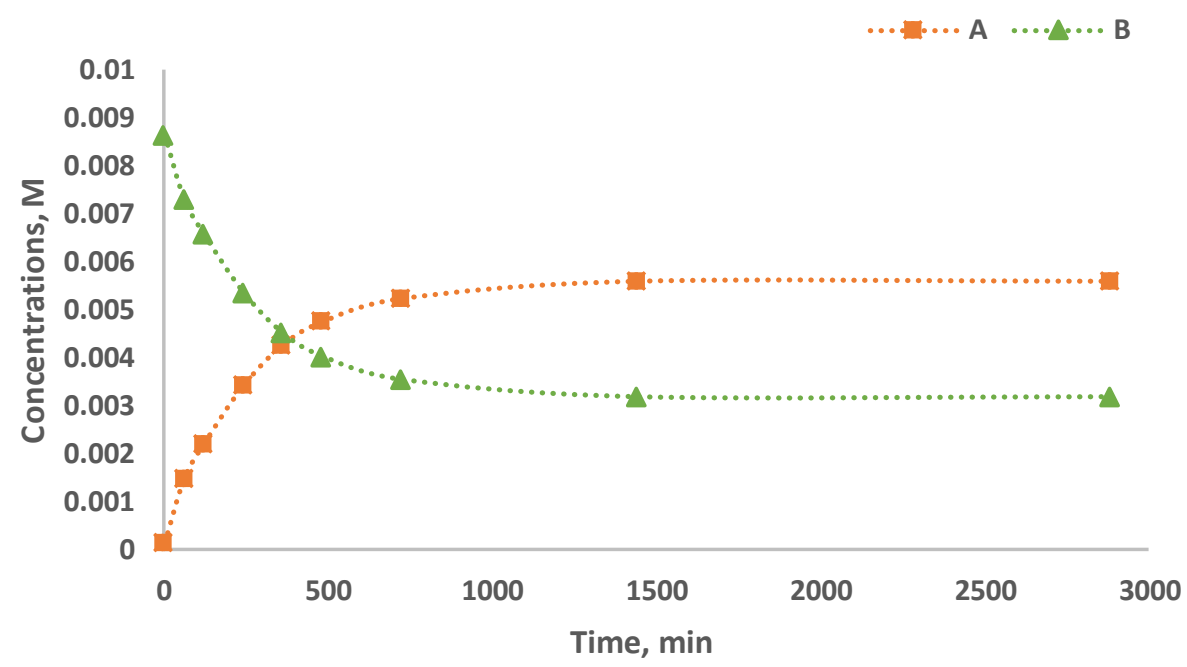

Figure S6. Plot of (A) $\left.\left[{ }^{(\mathrm{BBu} P O C O P}\right) \operatorname{Ir}\left(\mathrm{NCCH}_{3}\right)\right]$ and (B) $\left.\left[{ }^{\mathrm{t} B u}{ }^{\mathrm{POCOP}}\right) \operatorname{Ir}\left(\mathrm{PPh}_{3}\right)\right]$ vs. time (min), using 4 equivalents of $\mathrm{MeCN}$. The dotted lines added as a visual aid. 


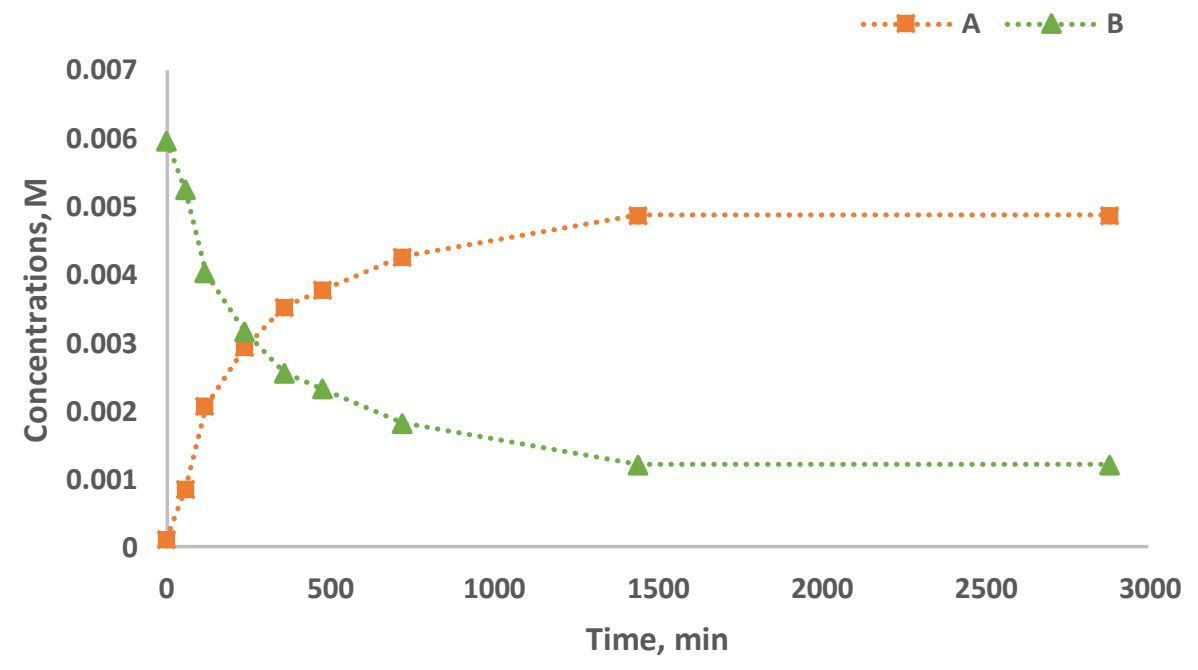

Figure S7. Plot of (A) [( $\left.\left.{ }^{\mathrm{tBu} P O C O P}\right) \operatorname{Ir}\left(\mathrm{NCCH}_{3}\right)\right]$ and (B) $\left[\left({ }^{\mathrm{tBu} P O C O P}\right) \operatorname{Ir}\left(\mathrm{PPh}_{3}\right)\right]$ vs. time $(\mathrm{min})$, using 8 equivalents of $\mathrm{MeCN}$. The dotted lines added as a visual aid.

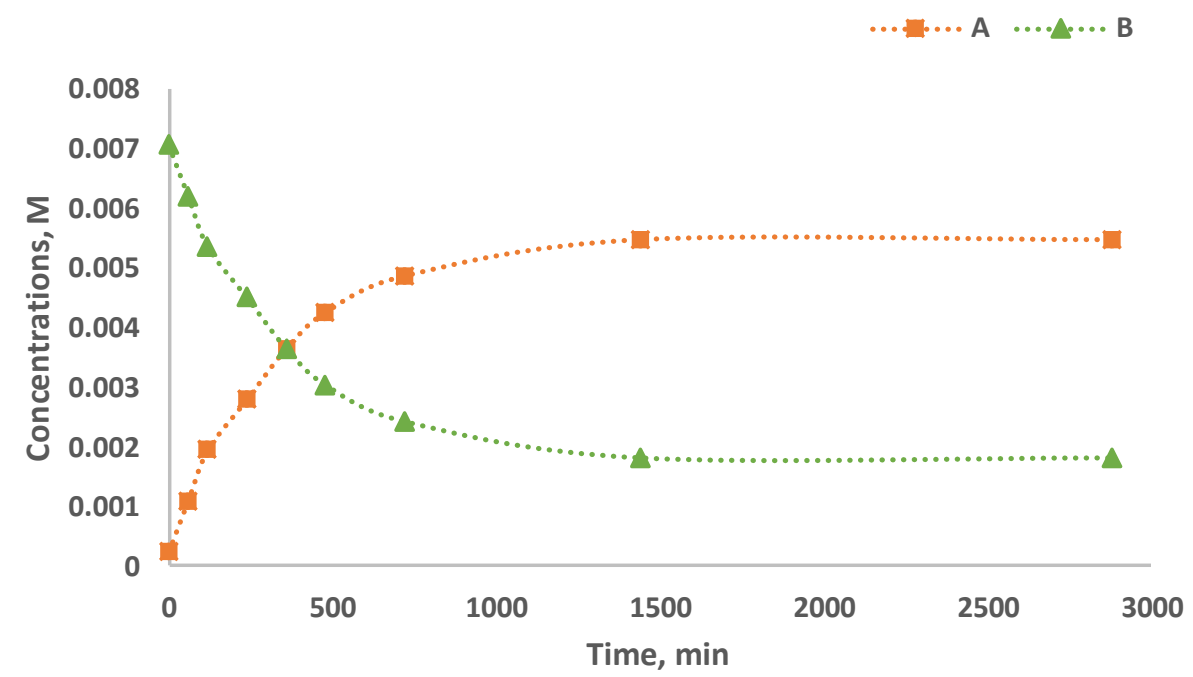

Figure S8. Plot of (A) [( $\left.\left.{ }^{\mathrm{tBu} P O C O P}\right) \operatorname{Ir}\left(\mathrm{NCCH}_{3}\right)\right]$ and (B) $\left[\left({ }^{\mathrm{tBu} P O C O P}\right) \operatorname{Ir}\left(\mathrm{PPh}_{3}\right)\right]$ vs. time $(\mathrm{min})$, using 16 equivalents of $\mathrm{MeCN}$. The dotted lines added as a visual aid. 
6. Table S1. Equilibrium constant and equilibrium $\Delta \mathrm{G}$ at $348.15 \mathrm{~K}$ determined by ${ }^{1} \mathrm{H}$ NMR in toluene- $d 8$

\begin{tabular}{|c|c|c|c|}
\hline POCOP complex & Incoming ligand & $K_{e q}(348.15 \mathrm{~K})$ & $\Delta G^{\circ} 348.15\left(\mathrm{kcal} \mathrm{mol}^{-1}\right)$ \\
\hline $\mathbf{4}$ & $\mathrm{Py}(2,4,8,16 \mathrm{eq})$ & 0.32 & 0.79 \\
\hline $\mathbf{4}$ & $\mathrm{MeCN}(2,4,8,16 \mathrm{eq})$ & 0.45 & 0.55 \\
\hline $\mathbf{3}$ & $\mathrm{PPh}_{3}(1 \mathrm{eq})$ & 3.80 & -0.92 \\
\hline $\mathbf{2}$ & $\mathrm{PPh}_{3}(1 \mathrm{eq})$ & 2.06 & -0.50 \\
\hline
\end{tabular}

7. Comparison of experimental and DFT calculations in terms of bond parameters and energy

Table S2. Comparison of solid state and DFT optimized geometries

\begin{tabular}{|c|c|c|}
\hline Structural parameters & Crystal structure & Geometry optimized \\
\hline $\operatorname{Ir}(1)-\mathrm{P}(2)^{\mathrm{a}}$ & $2.246(2) \AA$ & $2.276 \AA$ \\
\hline $\operatorname{Ir}(1)-\mathrm{P}(1)^{\mathrm{a}}$ & $2.246(2) \AA$ & $2.276 \AA$ \\
\hline $\mathrm{Ir}(1)-\mathrm{C}(40)^{\mathrm{a}}$ & $2.010(11) \AA$ & $2.022 \AA$ \\
\hline $\mathrm{C}(40)-\operatorname{Ir}(1)-\mathrm{P}(3)^{\mathrm{a}}$ & $178.90^{\circ}(4)$ & $178.44^{\circ}$ \\
\hline $\mathrm{P}(1)-\operatorname{Ir}(1)-\mathrm{P}(2)^{\mathrm{a}}$ & $159.40^{\circ}(15)$ & $1.99 \AA$ \\
\hline $\operatorname{Ir}(1)-\mathrm{N}(1)^{\mathrm{a}}$ & $2.054(10) \AA$ & $2.133 \AA$ \\
\hline $\operatorname{Ir}(1)-\mathrm{N}(1)^{\mathrm{b}}$ & $2.133(2) \AA$ & $2.283 \AA$ \\
\hline $\operatorname{Ir}(1)-\mathrm{P}(1)^{\mathrm{b}}$ & $2.256(6) \AA$ & $2.282 \AA$ \\
\hline $\operatorname{Ir}(1)-\mathrm{P}(2)^{\mathrm{b}}$ & $2.255(6) \AA$ & $2.019 \AA$ \\
\hline $\operatorname{Ir}(1)-\mathrm{C}(1)^{\mathrm{b}}$ & $2.004(3) \AA$ & $179.91^{\circ}$ \\
\hline $\mathrm{C}(39)-\operatorname{Ir}(1)-\mathrm{P}(3)^{\mathrm{b}}$ & $178.8^{\circ}(9)$ & $158.14^{\circ}$ \\
\hline $\mathrm{P}(1)-\operatorname{Ir}(1)-\mathrm{P}(2)^{\mathrm{b}}$ & $158.92^{\circ}(2)$ & $\mathrm{P}) 1 \mathrm{Py})]$ \\
\hline
\end{tabular}

${ }^{\mathrm{a}}$ is for [( $\left.\left.{ }^{\mathrm{tBu} P O C O P}\right) \operatorname{Ir}\left(\mathrm{NCCH}_{3}\right)\right]$ and ${ }^{\mathrm{b}}$ is for [( $\left.\left.{ }^{\mathrm{tBu}} \mathrm{POCOP}\right) \operatorname{Ir}(\mathrm{Py})\right]$. 
Table S3. Energy Values for Reaction w.r.t. SVP, TZVP, TZVPP basis set (in kcalmol-1)

\begin{tabular}{|c|c|c|c|}
\hline Reaction & $\Delta \mathrm{G}$ (SVP) & $\Delta \mathrm{G}(\mathrm{TZVP})$ & $\Delta \mathrm{G}$ (TZVPP) \\
\hline $\begin{array}{c}\text { Displacement of PPh3 with MeCN } \\
\text { (Scheme 2 manuscript) }\end{array}$ & 2.294 & 3.295 & 4.231 \\
\hline $\begin{array}{c}\text { Displacement of PPh3 with pyridine } \\
\text { (Scheme 2 manuscript) }\end{array}$ & 4.695 & 5.788 & 6.060 \\
\hline
\end{tabular}

Table S4. Energy barriers for associative mechanism.
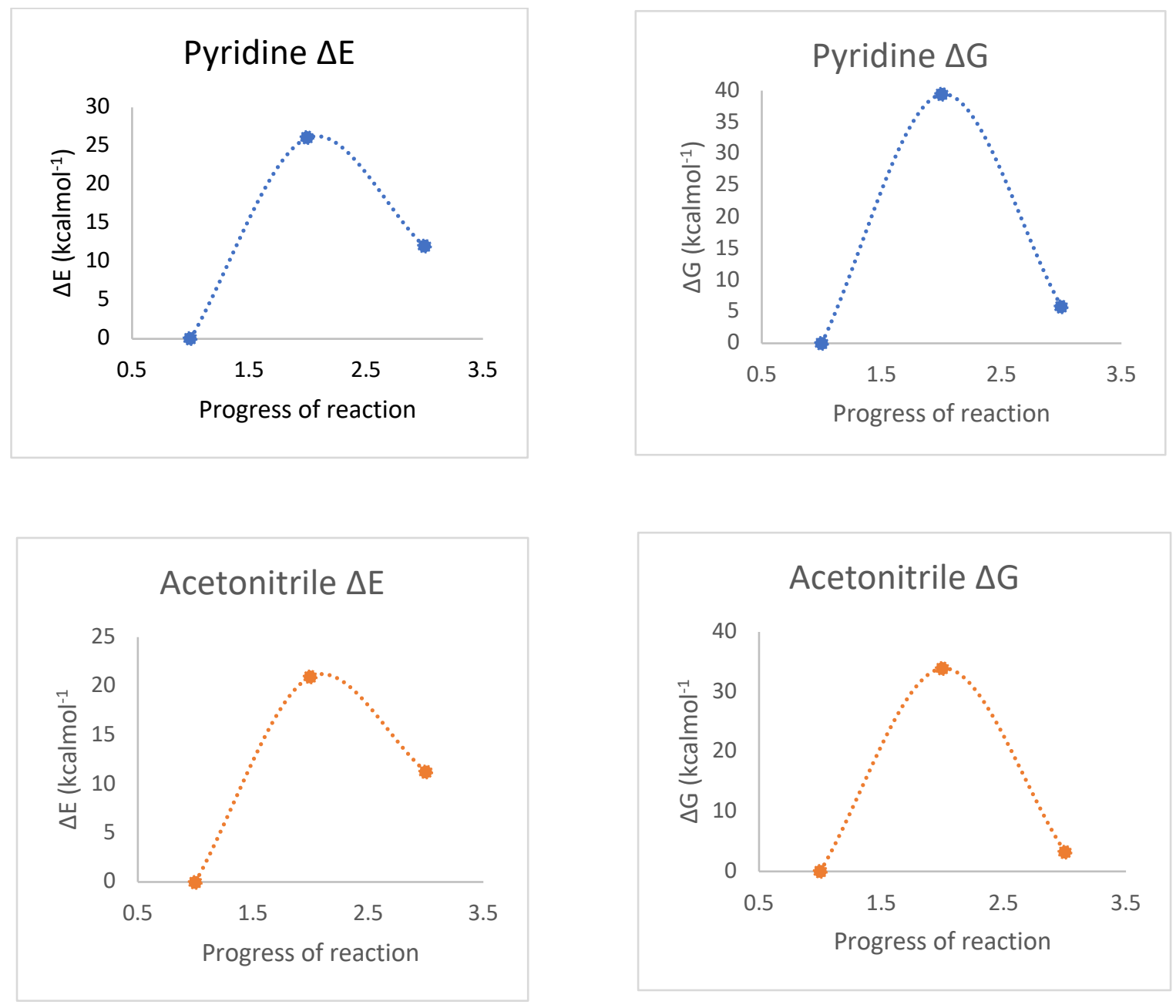


\section{Crystal data and structure refinement for $\left({ }^{\mathrm{tBu}} \mathrm{POCOP}\right) \operatorname{Ir}\left(\mathrm{NCCH}_{3}\right)$}

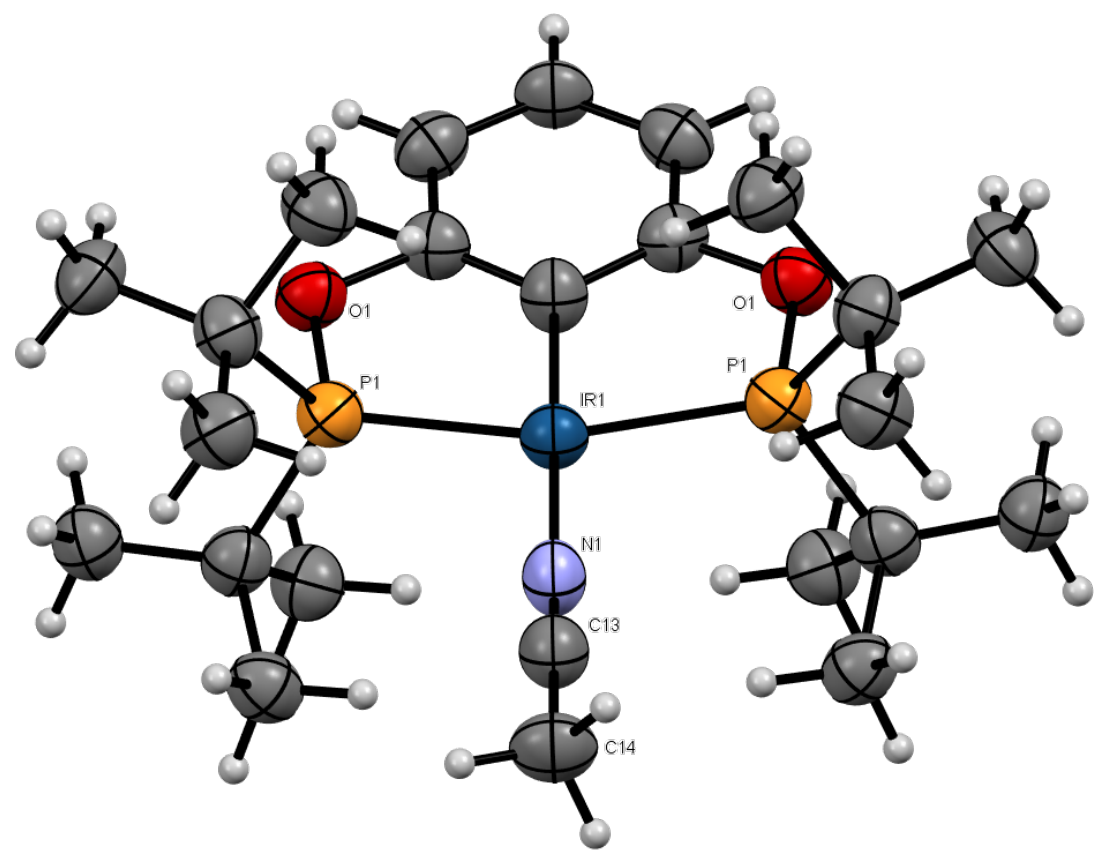

Figure S9. The thermal ellipsoids are represented at 50\% probability. Carbon, hydrogen, nitrogen, oxygen, phosphorus, and iridium atoms are represented by gray, white, light blue, red, light orange and magenta ellipsoids, respectively.

Crystal data and structure refinement for ( $\left.{ }^{\mathrm{tBu}} \mathrm{POCOP}\right) \operatorname{Ir}\left(\mathrm{NCCH}_{3}\right)$.

$\begin{array}{cc}\begin{array}{c}\text { Identification code } \\ \text { Crystal Color }\end{array} & \begin{array}{c}\text { fin19_31 } \\ \text { orange }\end{array} \\ \text { Crystal Habit } & \text { irregular } \\ \text { Empirical formula } & \text { C24 H42 Ir N O2 P2 } \\ \text { Formula weight } & 630.72 \\ \text { Temperature } & 100(2) \mathrm{K} \\ \text { Wavelength } & 1.54178 \AA \\ \text { Crystal system } & \text { Orthorhombic } \\ \text { Space group } & \text { Pbcm } \\ \text { a cell dimensions } & \mathrm{a}=9.1137(2) \AA \text { alpha }=90^{\circ} . \\ & \mathrm{b}=14.3331(3) \AA \text { beta }=90^{\circ} .\end{array}$




$$
\mathrm{c}=20.2294(5) \AA \text { gamma }=90^{\circ} \text {. }
$$

Volume

Z

Calculated density

Absorption coefficient

$\mathrm{F}(000)$
2642.52(10) $\AA^{3}$

4

$1.585 \mathrm{Mg} / \mathrm{m}^{3}$

$11.069 \mathrm{~mm}^{-1}$

1264

Crystal size

$0.130 \times 0.068 \times 0.038 \mathrm{~mm}$

Theta range for data collection

4.371 to $77.434^{\circ}$.

Limiting indices

$-10<=\mathrm{h}<=11,-17<=\mathrm{k}<=18,-24<=\mathrm{l}<=25$

Reflections collected / unique

$4622 / 4622[\mathrm{R}(\mathrm{int})=$ ?]

Completeness to theta $=67.679^{\circ}$

$99.7 \%$

Refinement method

Full-matrix least-squares on $\mathrm{F}^{2}$

Data / restraints / parameters

4622 / 0 / 154

Goodness-of-fit on $\mathrm{F}^{2}$

1.113

Final $\mathrm{R}$ indices $[\mathrm{I}>2 \operatorname{sigma}(\mathrm{I})]$

$\mathrm{R} 1=0.0888, \mathrm{wR} 2=0.2618$

$\mathrm{R}$ indices (all data)

$\mathrm{R} 1=0.0957, \mathrm{wR} 2=0.2759$

Extinction coefficient

$0.0007(2)$

Largest diff. peak and hole

4.787 and -3.303 e. $\AA^{-3}$ 


\section{Crystal data and structure refinement for ( $\left.{ }^{\mathrm{B} u} \mathrm{POCOP}\right) \operatorname{Ir}(\mathrm{Py})$}

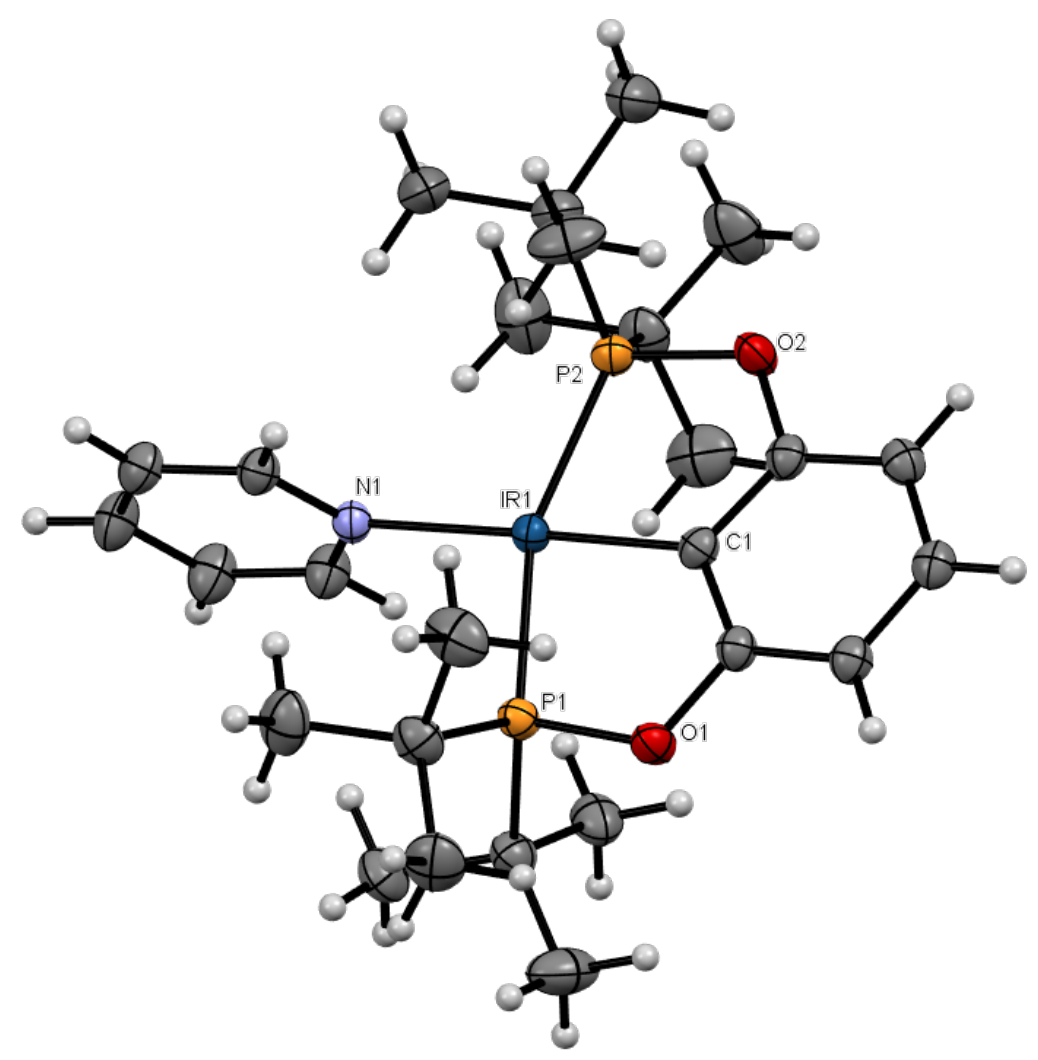

Figure S10. The thermal ellipsoids are represented at 50\% probability. Carbon, hydrogen, nitrogen, oxygen, phosphorus, and iridium atoms are represented by gray, white, light blue, red, orange, and purple ellipsoids, respectively. The interstitial pentane molecule was omitted for clarity.

Crystal data and structure refinement for ( $\left.{ }^{\mathrm{tBu} P O C O P}\right) \operatorname{Ir}(\mathrm{Py})$.

Crystal Color

Crystal Habit

Empirical formula

Formula weight

Temperature

Wavelength

Crystal system orange

irregular

C29.50 H50 Ir N O2 P2

704.84

100(2) K

$1.54178 \AA$

Monoclinic 
Space group

Unit cell dimensions

$$
\begin{gathered}
\mathrm{a}=9.36583(5) \AA \text { alpha }=90^{\circ} . \\
\mathrm{b}=15.00626(10) \AA \quad \text { beta }=95.2309(5)^{\circ} . \\
\mathrm{c}=22.75188(12) \AA \text { gamma }=90^{\circ} .
\end{gathered}
$$

Volume

Z

Calculated density

Absorption coefficient

$\mathrm{F}(000)$

$$
\text { 3184.37(3) } \AA^{3}
$$

4

$1.470 \mathrm{Mg} / \mathrm{m}^{3}$

$9.248 \mathrm{~mm}^{-1}$

1428

Crystal size

$0.183 \times 0.115 \times 0.085 \mathrm{~mm}$

Theta range for data collection

3.533 to $77.450^{\circ}$.

Limiting indices

$-8<=\mathrm{h}<=11,-18<=\mathrm{k}<=18,-28<=1<=28$

Reflections collected / unique

$57395 / 6708[\mathrm{R}($ int $)=0.0408]$

Completeness to theta $=67.679^{\circ} \quad 99.9 \%$

Refinement method

Full-matrix least-squares on $\mathrm{F}^{2}$

Data / restraints / parameters

6708 / 24 / 357

Goodness-of-fit on $\mathrm{F}^{2}$

\subsection{3}

Final $R$ indices $[\mathrm{I}>2 \operatorname{sigma}(\mathrm{I})]$

$\mathrm{R} 1=0.0243, \mathrm{wR} 2=0.0649$

$\mathrm{R}$ indices (all data)

$\mathrm{R} 1=0.0257, \mathrm{wR} 2=0.0660$

Largest diff. peak and hole

0.821 and -1.016 e. $\AA$ 
10. Representative ${ }^{1} \mathrm{H},{ }^{31} \mathrm{P}$ NMRs for displacement of $\mathrm{PPh}_{3}$ by pyridine after equilibrium generation

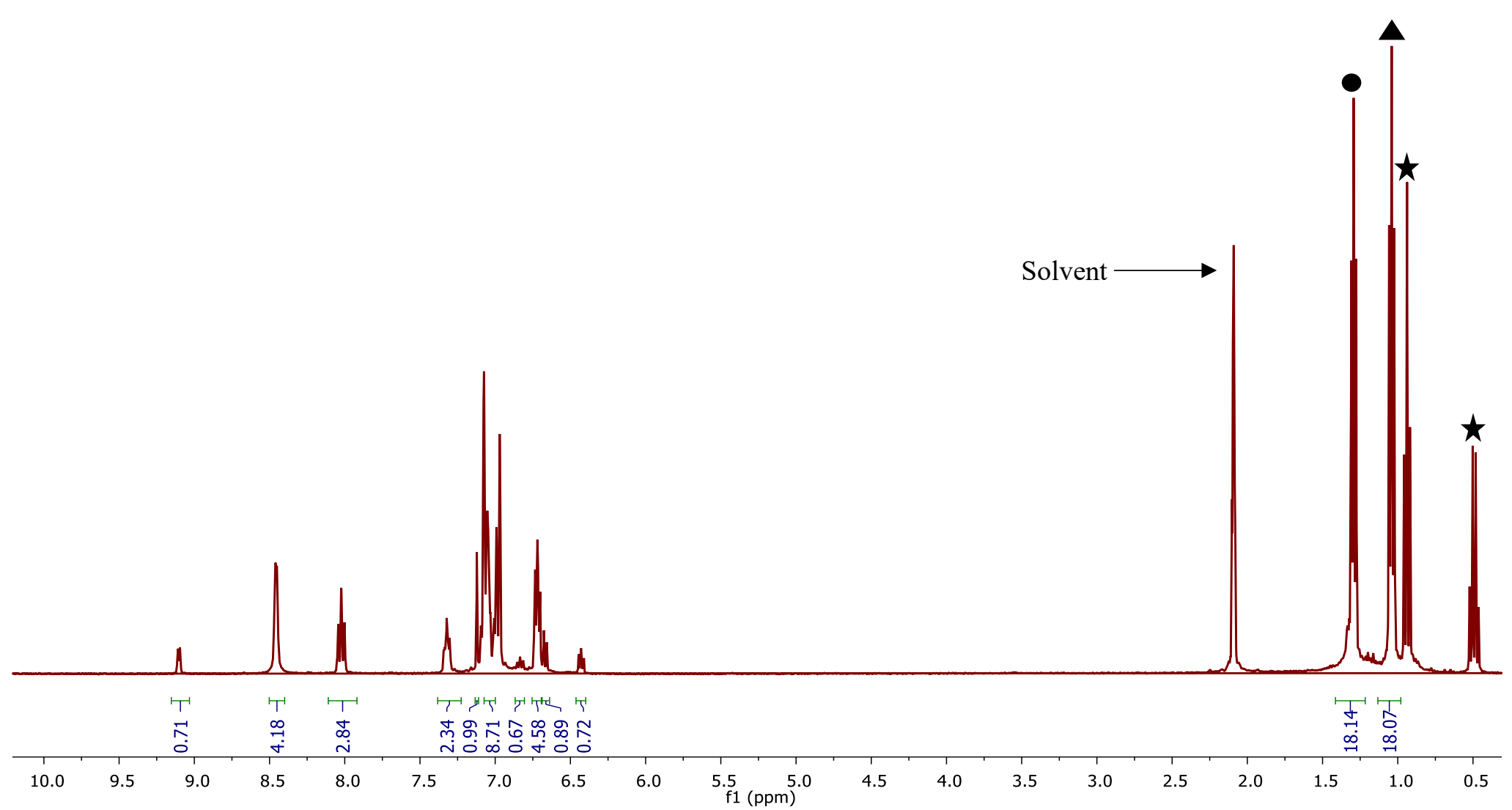

Figure S10. ${ }^{1} \mathrm{H}$ NMR of ( $\left.{ }^{\mathrm{tBu}} \mathrm{POCOP}\right) \operatorname{Ir}\left(\mathrm{PPh}_{3}\right)$ and $\left({ }^{\mathrm{tBu}} \mathrm{POCOP}\right) \operatorname{Ir}(\mathrm{Py})$ equilibrium after $24 \mathrm{~h}$, using 2 equivalents of pyridine. represents ( $\left.{ }^{\mathrm{B} B u} \mathrm{POCOP}\right) \operatorname{Ir}(\mathrm{Py}),(\boldsymbol{\bullet})$ represents $\left({ }^{\mathrm{tBu} P O C O P}\right) \operatorname{Ir}\left(\mathrm{PPh}_{3}\right)$ and $(\boldsymbol{\bullet})$ represents internal standard. 

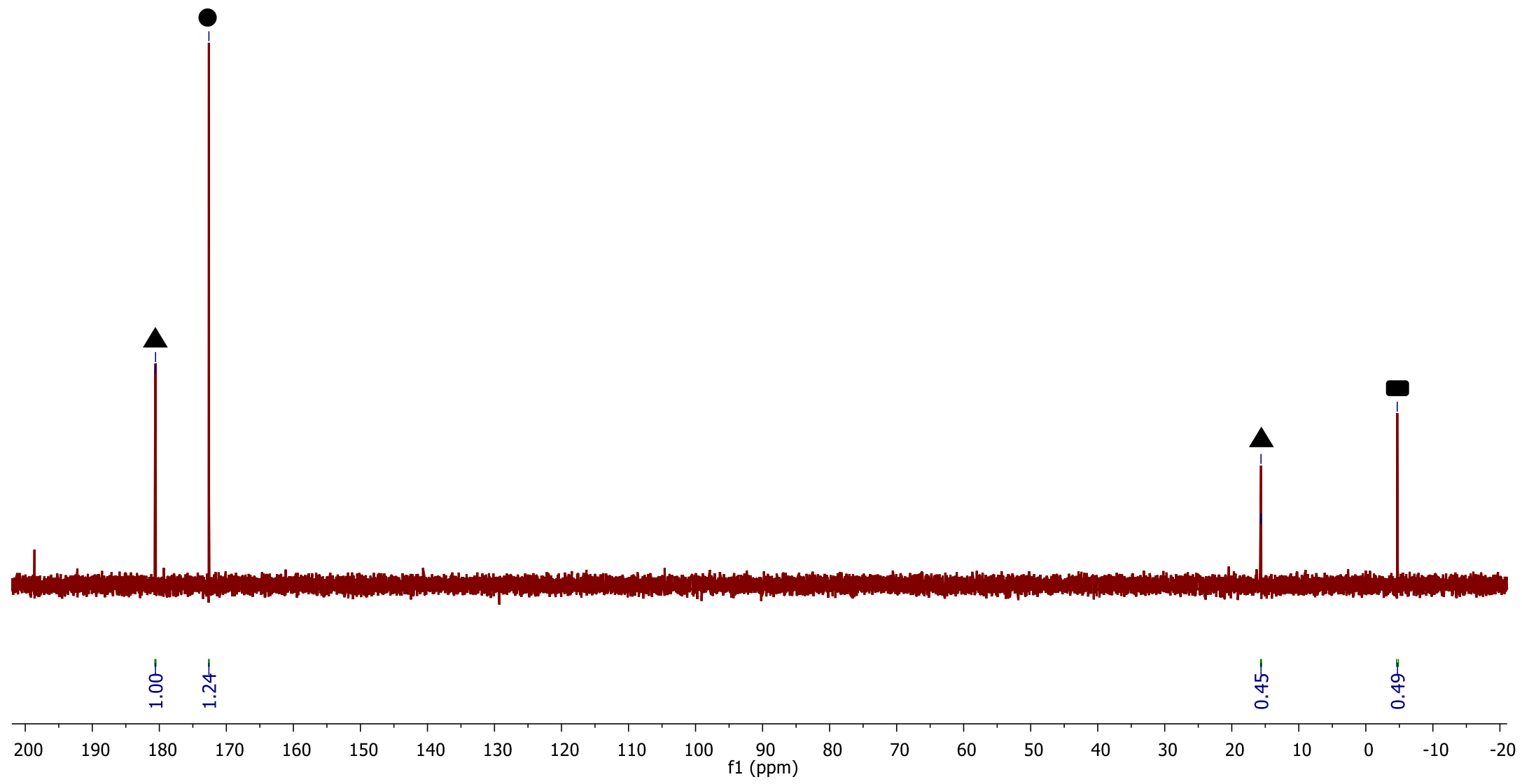

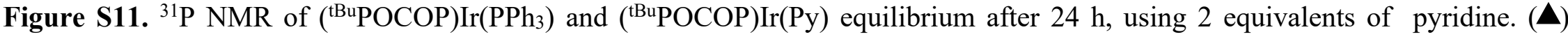
represents ( $\left.{ }^{\mathrm{tBu}} \mathrm{POCOP}\right) \operatorname{Ir}\left(\mathrm{PPh}_{3}\right),(\bullet)$ represents ( $\left.{ }^{\mathrm{tBu}} \mathrm{POCOP}\right) \operatorname{Ir}(\mathrm{Py})$ and $(\boldsymbol{\square})$ represents $\mathrm{PPh}_{3}$. 


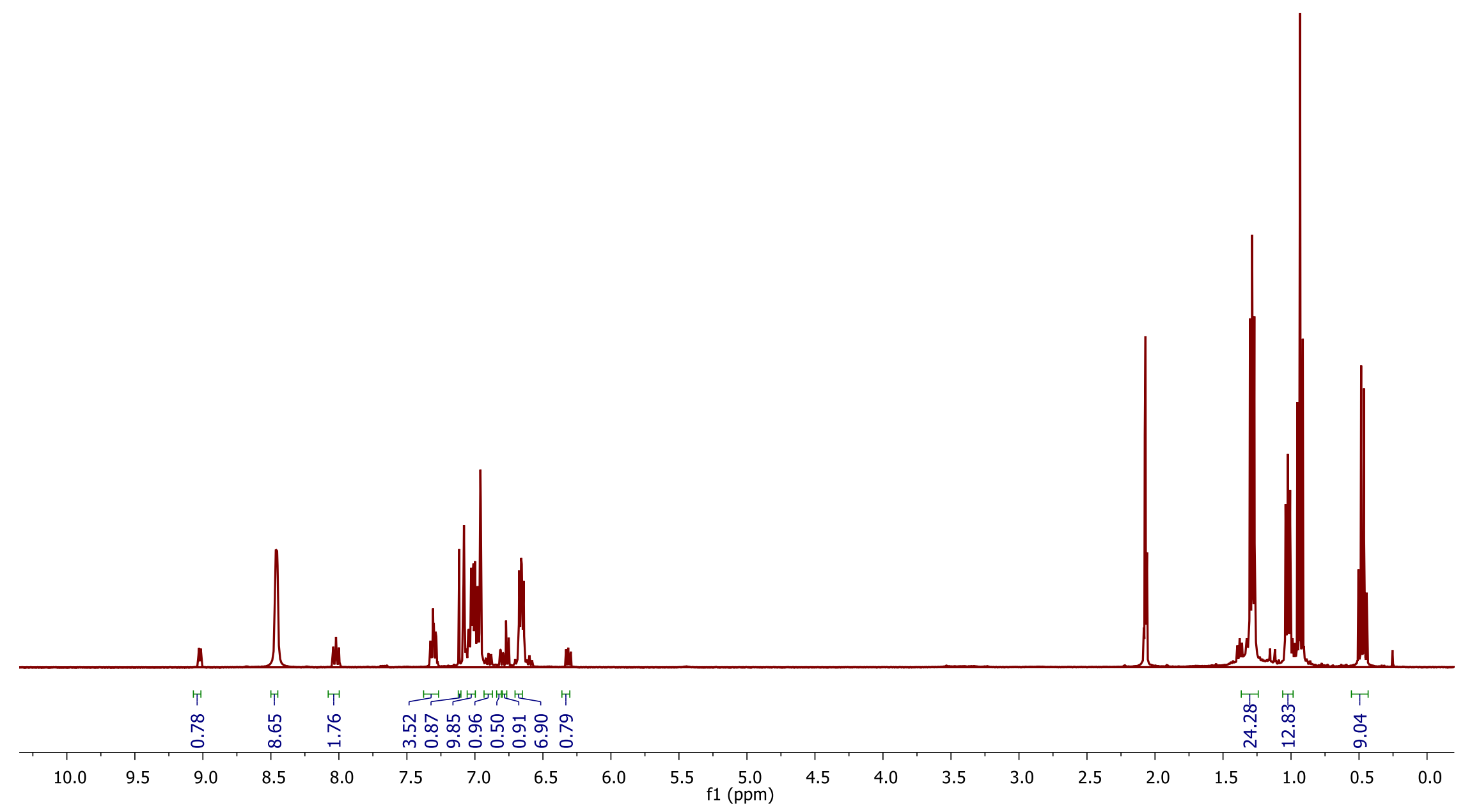

Figure S12. ${ }^{1} \mathrm{H}$ NMR of $\left({ }^{\mathrm{tBu}} \mathrm{POCOP}\right) \operatorname{Ir}\left(\mathrm{PPh}_{3}\right)$ and $\left({ }^{\mathrm{tBu}} \mathrm{POCOP}\right) \operatorname{Ir}(\mathrm{Py})$ equilibrium after $24 \mathrm{~h}$, using 4 equivalents of pyridine. 


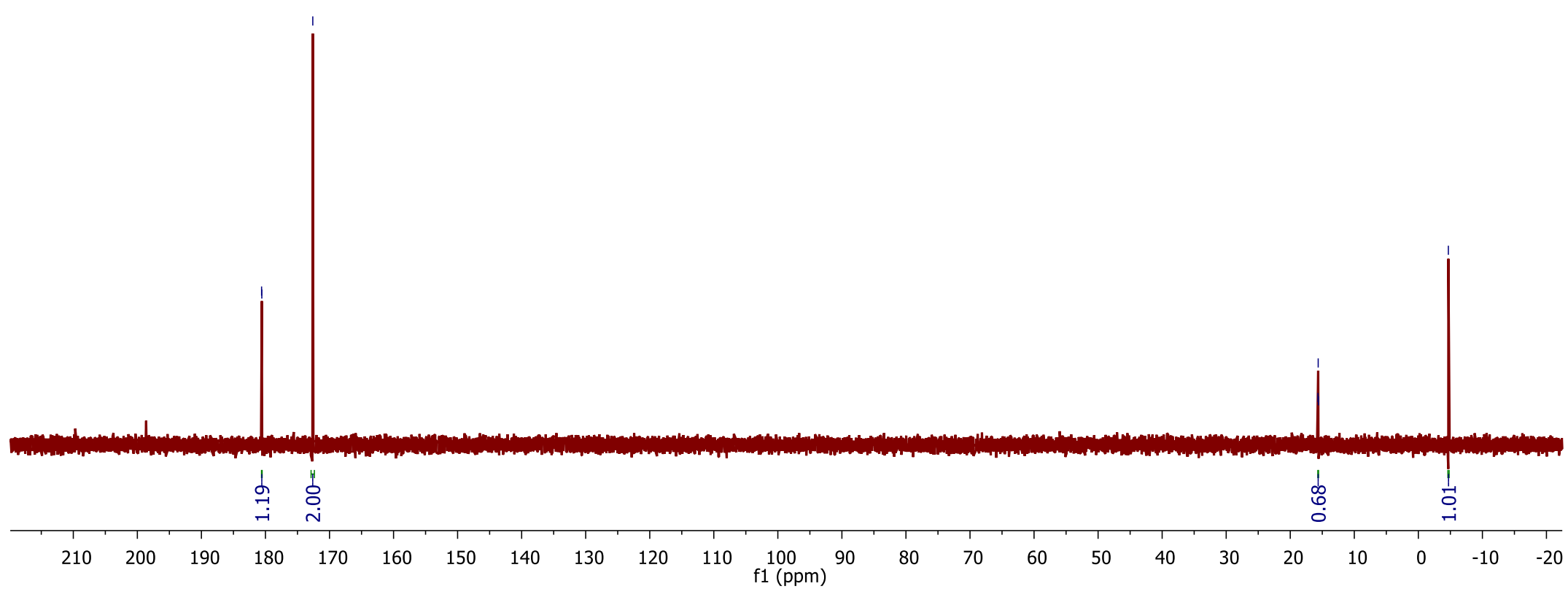

Figure S13. ${ }^{31} \mathrm{P}$ NMR of ( $\left.{ }^{\mathrm{tBu}} \mathrm{POCOP}\right) \operatorname{Ir}\left(\mathrm{PPh}_{3}\right)$ and $\left({ }^{\mathrm{tBu}} \mathrm{POCOP}\right) \operatorname{Ir}(\mathrm{Py})$ equilibrium after $24 \mathrm{~h}$, using 4 equivalents of pyridine. 


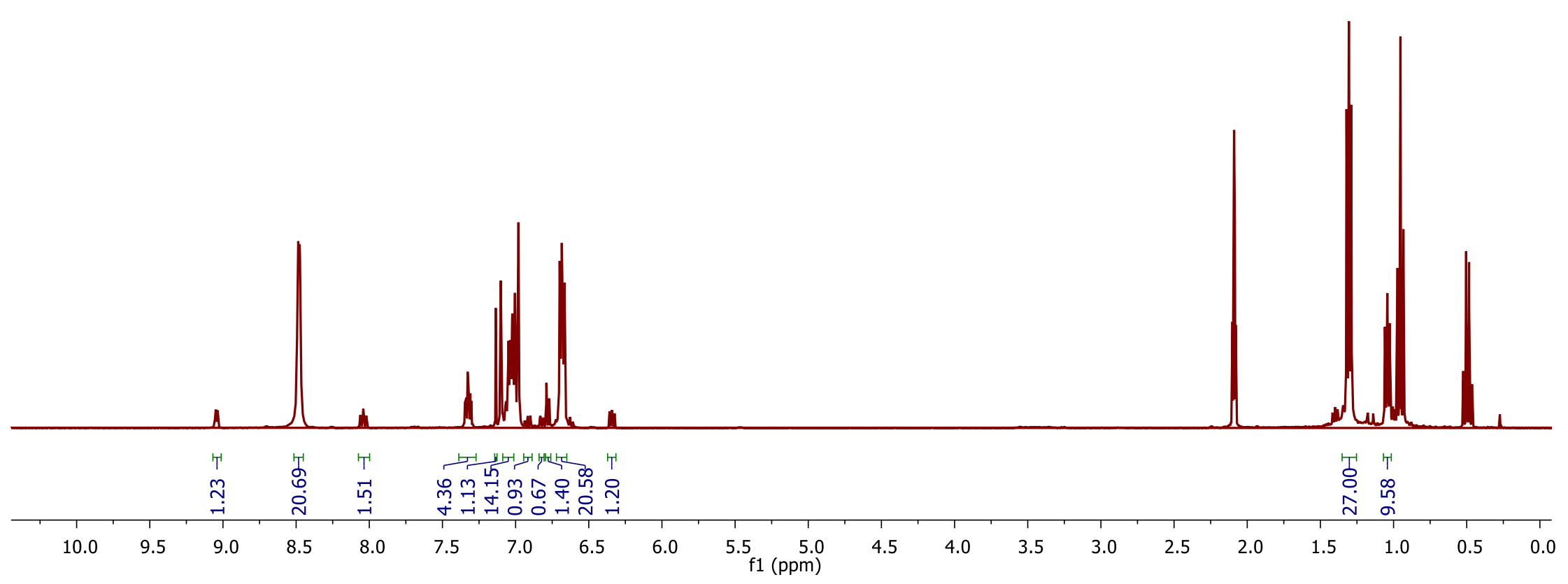

Figure S14. ${ }^{1} \mathrm{H}$ NMR of ( $\left.{ }^{\mathrm{B} B}{ }^{\mathrm{POOCOP}}\right) \operatorname{Ir}\left(\mathrm{PPh}_{3}\right)$ and ( $\left.{ }^{\mathrm{tBu}} \mathrm{POCOP}\right) \operatorname{Ir}(\mathrm{Py})$ equilibrium after $24 \mathrm{~h}$, using 8 equivalents of pyridine. 


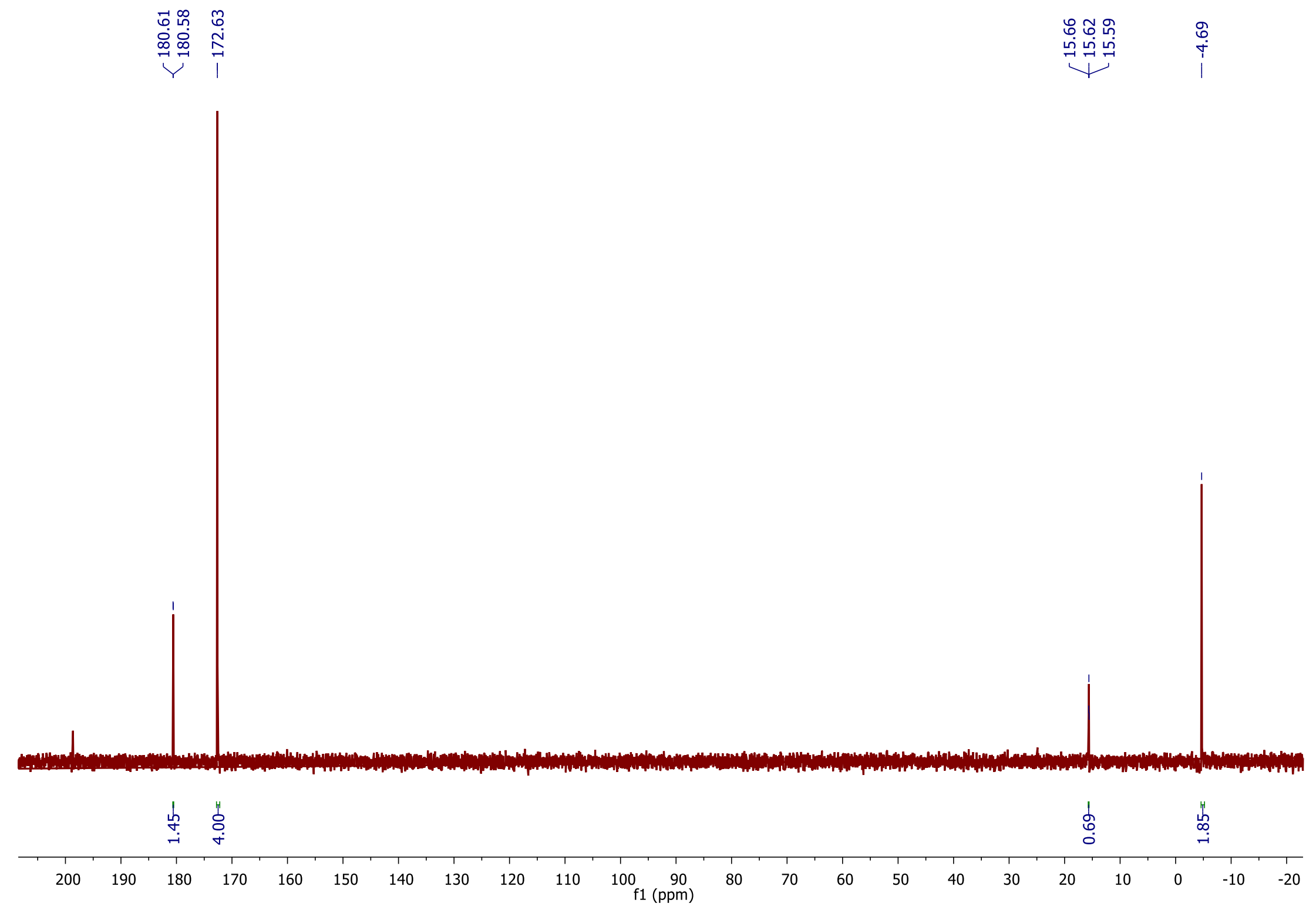

Figure S15. ${ }^{31} \mathrm{P}$ NMR of ( $\left.{ }^{\mathrm{tBu} P O C O P}\right) \operatorname{Ir}\left(\mathrm{PPh}_{3}\right)$ and $\left({ }^{\mathrm{tBu}} \mathrm{POCOP}\right) \operatorname{Ir}(\mathrm{Py})$ equilibrium after $24 \mathrm{~h}$, using 8 equivalents of pyridine. 


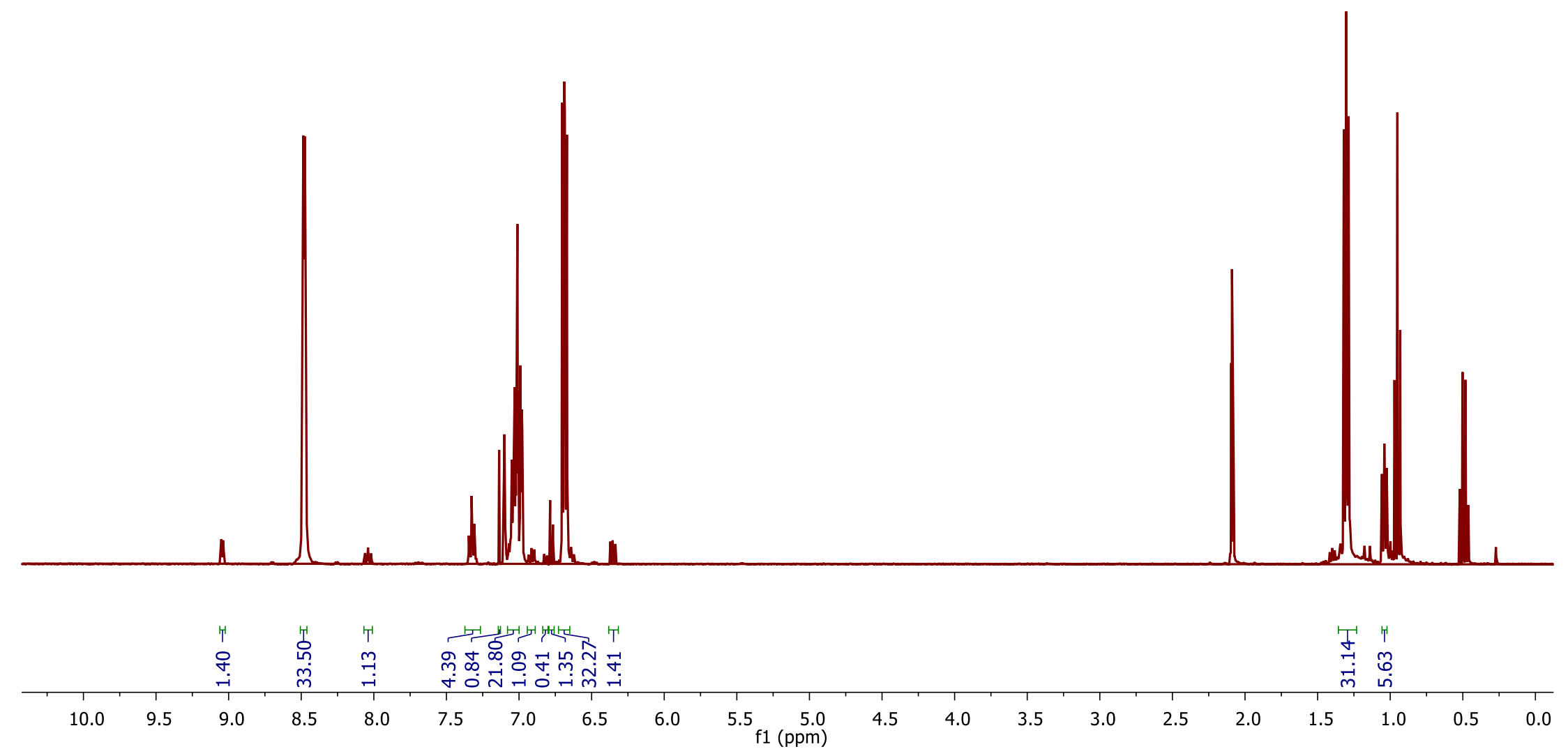

Figure S16. ${ }^{1} \mathrm{H}$ NMR of ( $\left.{ }^{\mathrm{tBu}} \mathrm{POCOP}\right) \operatorname{Ir}\left(\mathrm{PPh}_{3}\right)$ and $\left({ }^{\mathrm{tBu}} \mathrm{POCOP}\right) \operatorname{Ir}(\mathrm{Py})$ equilibrium after $24 \mathrm{~h}$, using 16 equivalents of pyridine. 


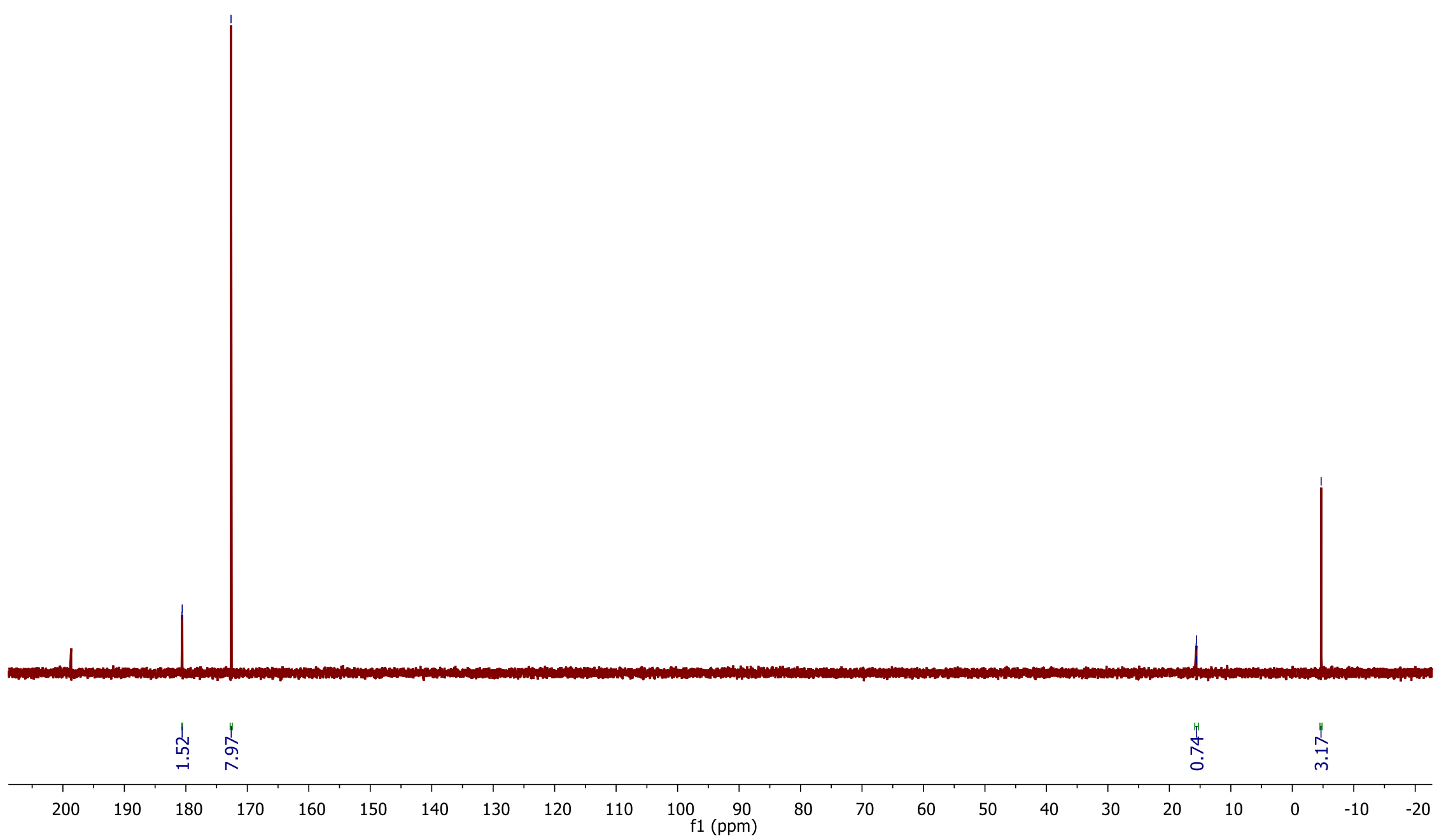

Figure S17. ${ }^{31} \mathrm{P}$ NMR of $\left({ }^{\mathrm{tBu}} \mathrm{POCOP}\right) \operatorname{Ir}\left(\mathrm{PPh}_{3}\right)$ and $\left({ }^{\mathrm{tBu}} \mathrm{POCOP}\right) \operatorname{Ir}(\mathrm{Py})$ equilibrium after $24 \mathrm{~h}$, using 16 equivalents of pyridine. 


\section{Representative ${ }^{1} \mathrm{H},{ }^{31} \mathrm{P}$ NMRs for displacement of $\mathrm{PPh}_{3}$ by acetonitrile after equilibrium generation}

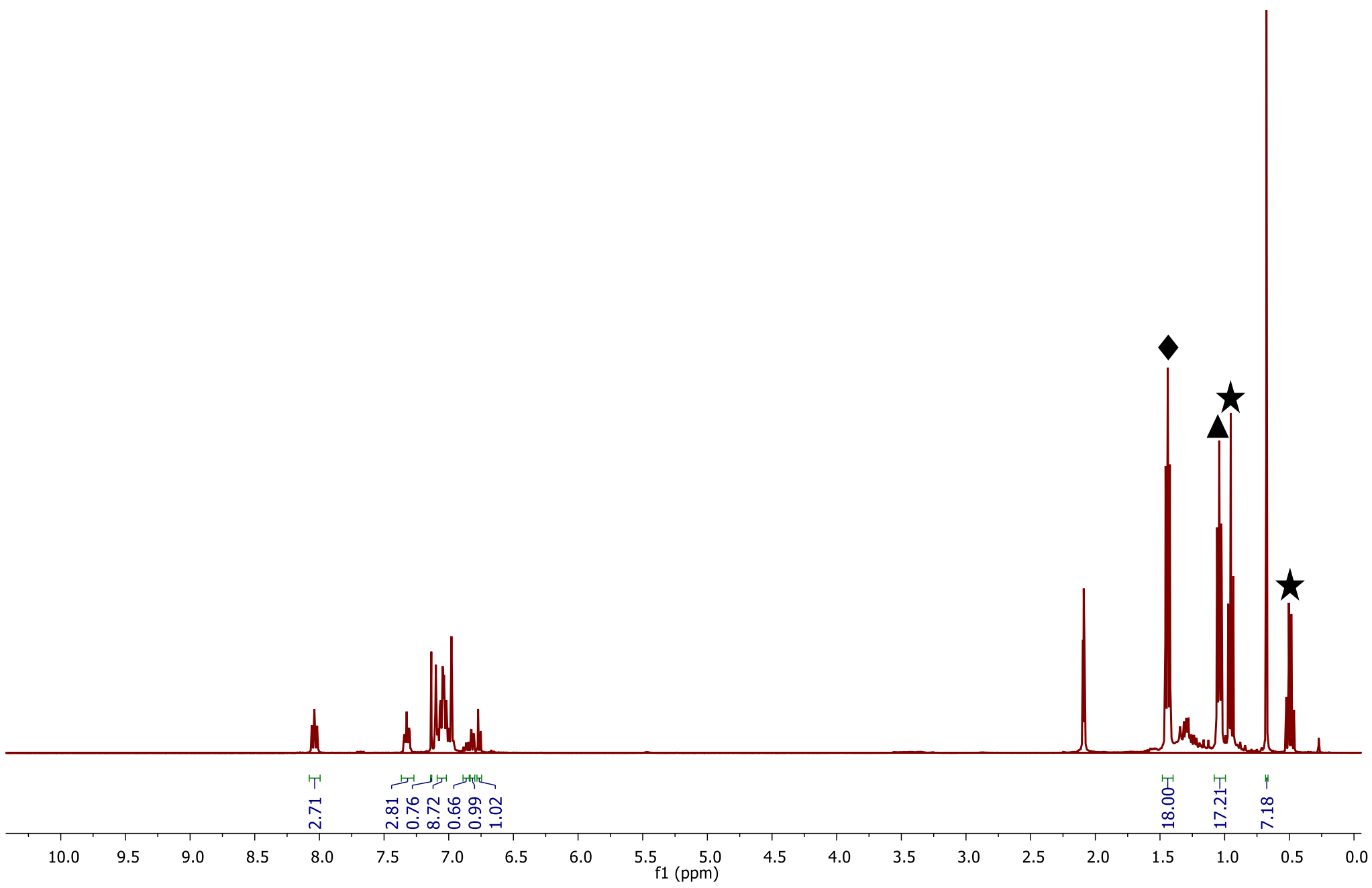

Figure S18. ${ }^{1} \mathrm{H}$ NMR of ( $\left.{ }^{\mathrm{tBu}} \mathrm{POCOP}\right) \operatorname{Ir}\left(\mathrm{PPh}_{3}\right)$ and $\left({ }^{\mathrm{tBu}} \mathrm{POCOP}\right) \operatorname{Ir}\left(\mathrm{NCCH}_{3}\right)$ equilibrium after 24h, using 2 equivalents of $\mathrm{MeCN}$. represents ( $\left.{ }^{\mathrm{tBu}} \mathrm{POCOP}\right) \operatorname{Ir}\left(\mathrm{NCCH}_{3}\right),(\mathbf{\Delta})$ represents ( $\left.{ }^{\mathrm{tBu}} \mathrm{POCOP}\right) \operatorname{Ir}\left(\mathrm{PPh}_{3}\right)$ and $(\boldsymbol{\star} \boldsymbol{\star})$ represents internal standard. 


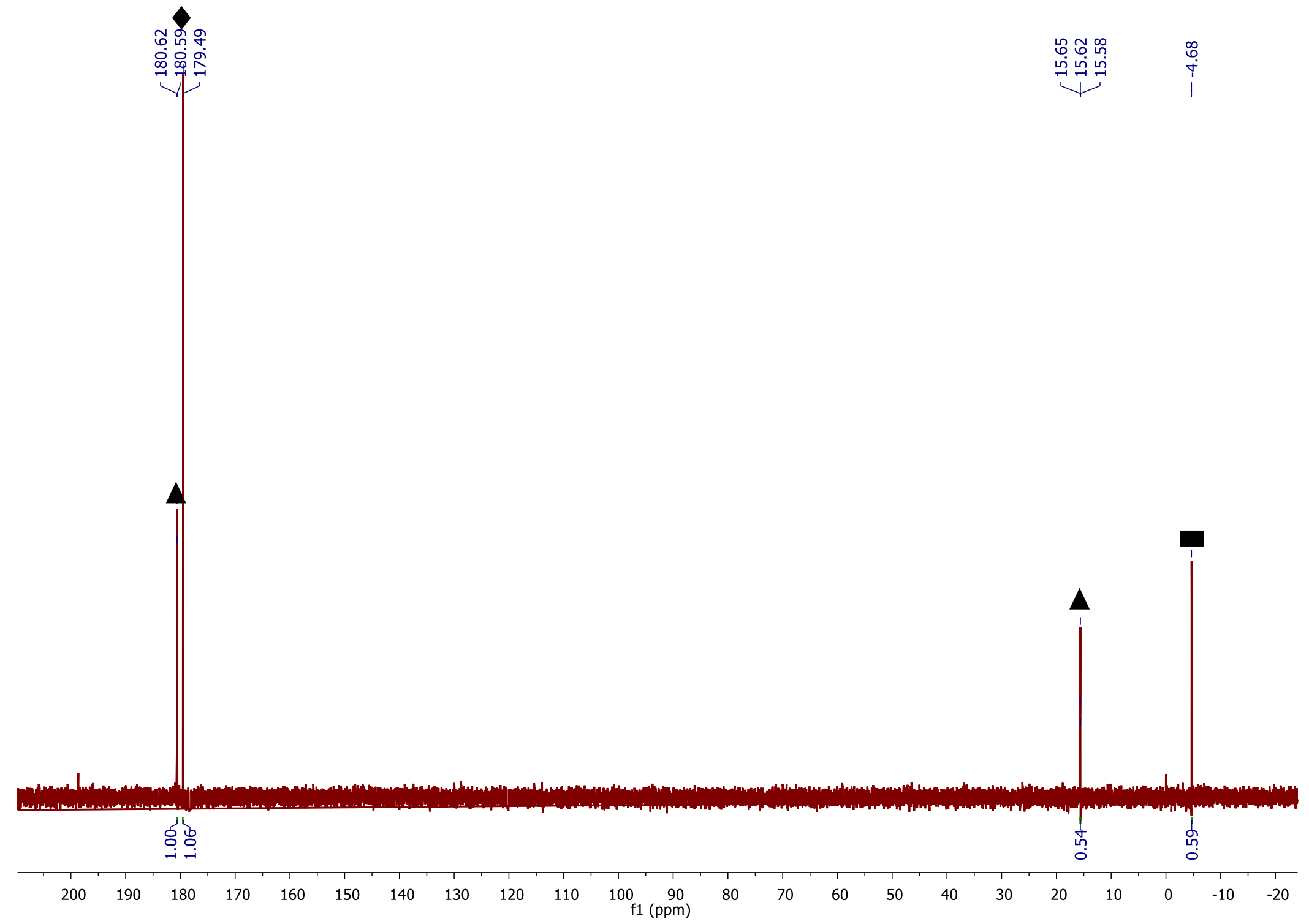

Figure S19. ${ }^{31} \mathrm{P}$ NMR of ( $\left.{ }^{\mathrm{tBu}} \mathrm{POCOP}\right) \operatorname{Ir}\left(\mathrm{PPh}_{3}\right)$ and $\left({ }^{\mathrm{tBu}} \mathrm{POCOP}\right) \operatorname{Ir}\left(\mathrm{NCCH}_{3}\right)$ equilibrium after $24 \mathrm{~h}$, using 2 equivalents of $\mathrm{MeCN}$. $(\mathbf{\Delta})$ represents ( $\left.{ }^{\mathrm{tBu}} \mathrm{POCOP}\right) \operatorname{Ir}\left(\mathrm{PPh}_{3}\right),(\boldsymbol{\vee})$ represents ( $\left.{ }^{\mathrm{tBu}} \mathrm{POCOP}\right) \operatorname{Ir}\left(\mathrm{NCCH}_{3}\right)$ and $(\mathbf{\square})$ represents $\mathrm{PPh}_{3}$. 


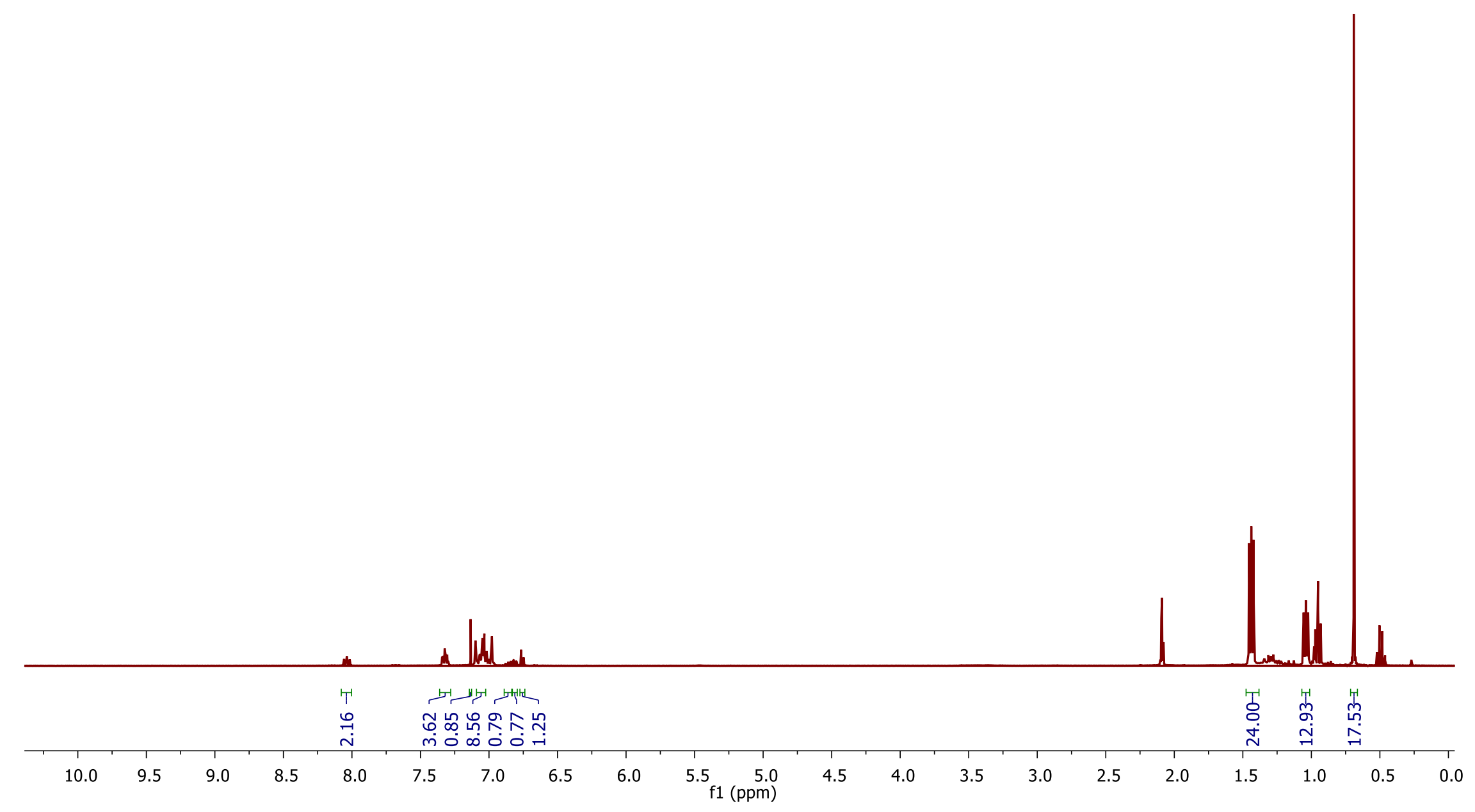

Figure S20. ${ }^{1} \mathrm{H}$ NMR of ( $\left.{ }^{\mathrm{tBu} P O C O P}\right) \operatorname{Ir}\left(\mathrm{PPh}_{3}\right)$ and $\left({ }^{\mathrm{tBu}} \mathrm{POCOP}\right) \operatorname{Ir}\left(\mathrm{NCCH}_{3}\right)$ equilibrium after $24 \mathrm{~h}$, using 4 equivalents of $\mathrm{MeCN}$. 


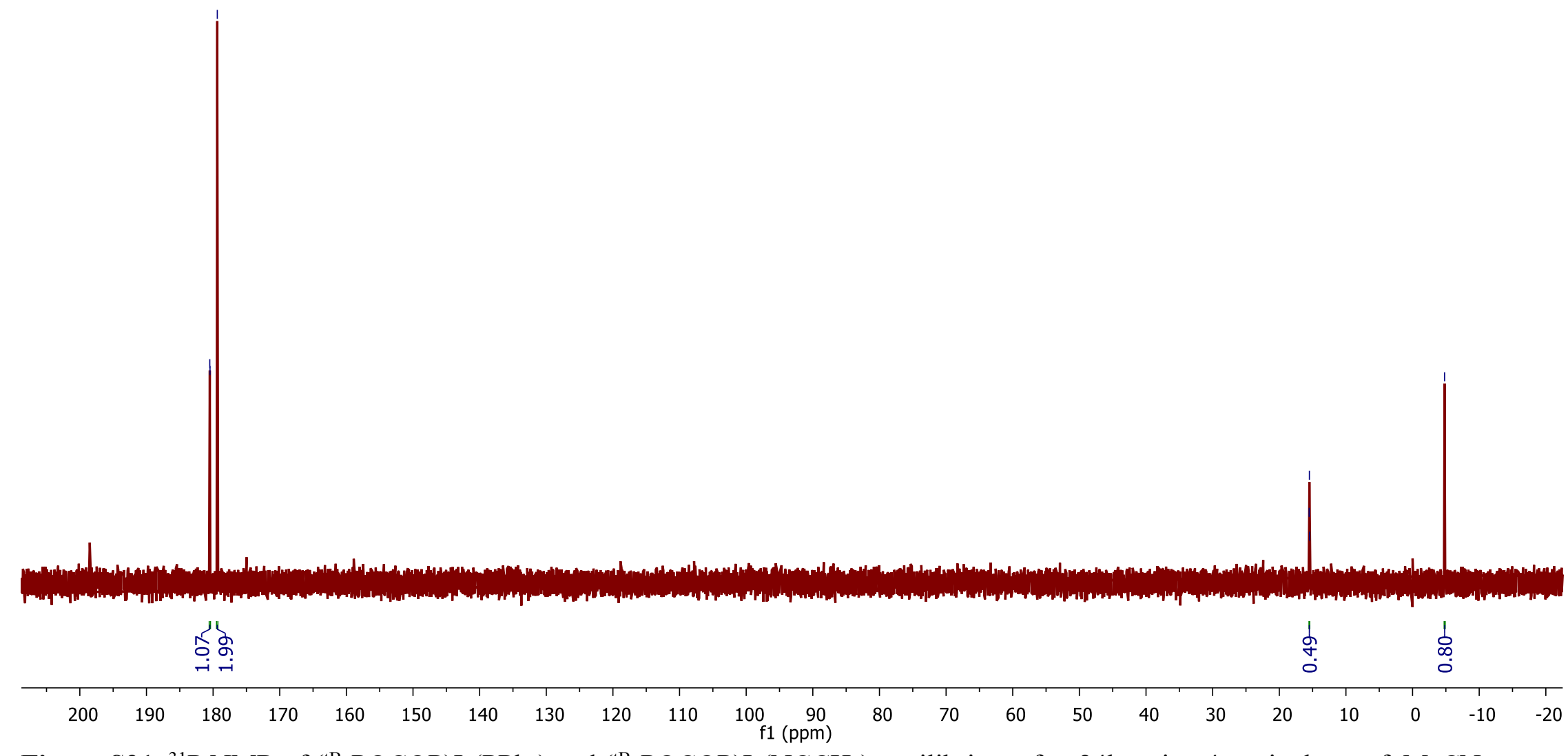

Figure S21. ${ }^{31} \mathrm{P}$ NMR of ( $\left.{ }^{\mathrm{tBu}} \mathrm{POCOP}\right) \operatorname{Ir}\left(\mathrm{PPh}_{3}\right)$ and ( $\left.{ }^{\mathrm{B} u} \mathrm{POCOP}\right) \operatorname{Ir}\left(\mathrm{NCCH}_{3}\right)$ equilibrium after $24 \mathrm{~h}$, using 4 equivalents of $\mathrm{MeCN}$. 


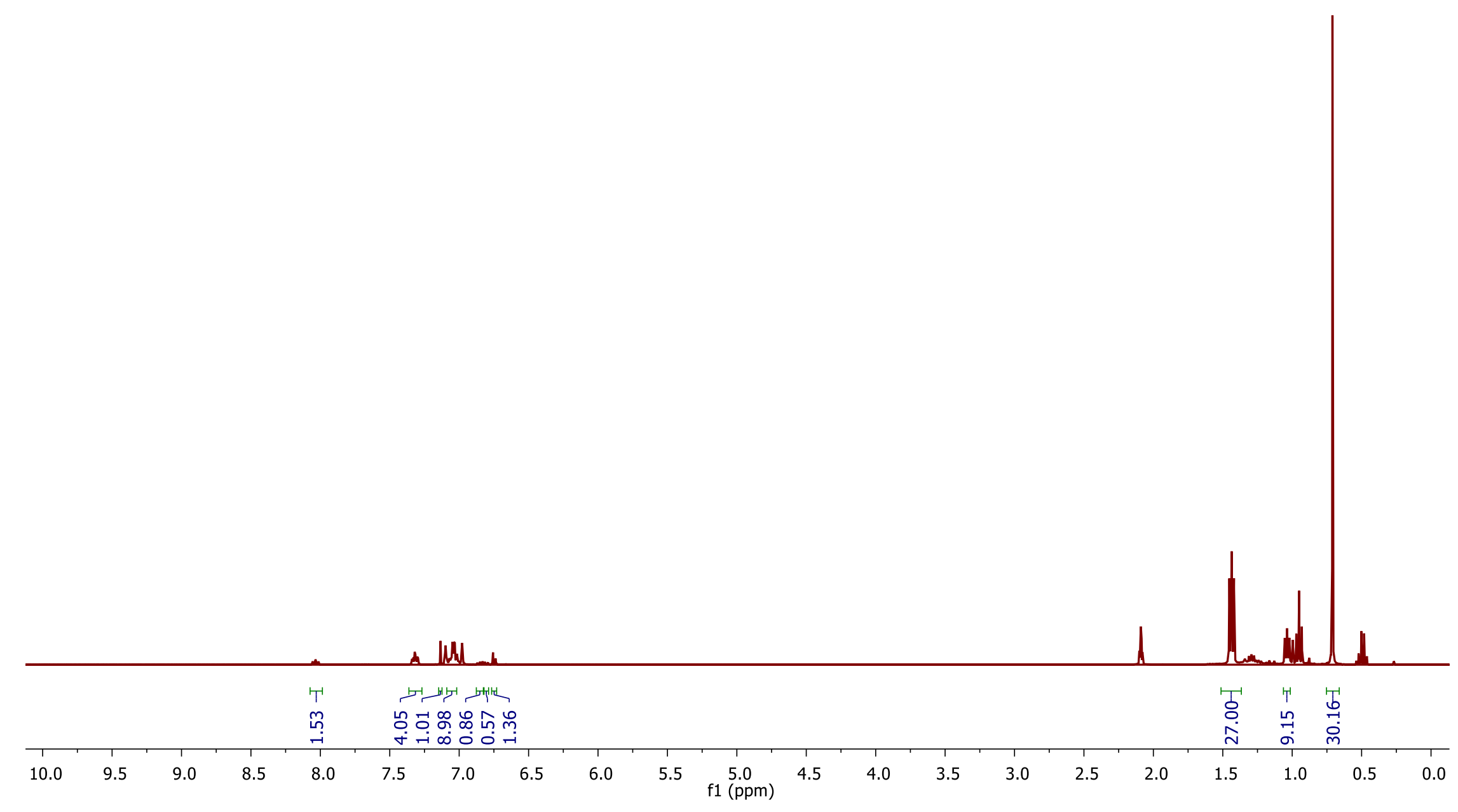

Figure S22. ${ }^{1} \mathrm{H}$ NMR of $\left({ }^{\mathrm{tBu}} \mathrm{POCOP}\right) \operatorname{Ir}\left(\mathrm{PPh}_{3}\right)$ and $\left({ }^{\mathrm{tBu}} \mathrm{POCOP}\right) \operatorname{Ir}\left(\mathrm{NCCH}_{3}\right)$ equilibrium after $24 \mathrm{~h}$, using 8 equivalents of $\mathrm{MeCN}$. 


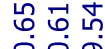

票密

ㅇํำํํำ

宊

$\stackrel{4}{i}$

VI

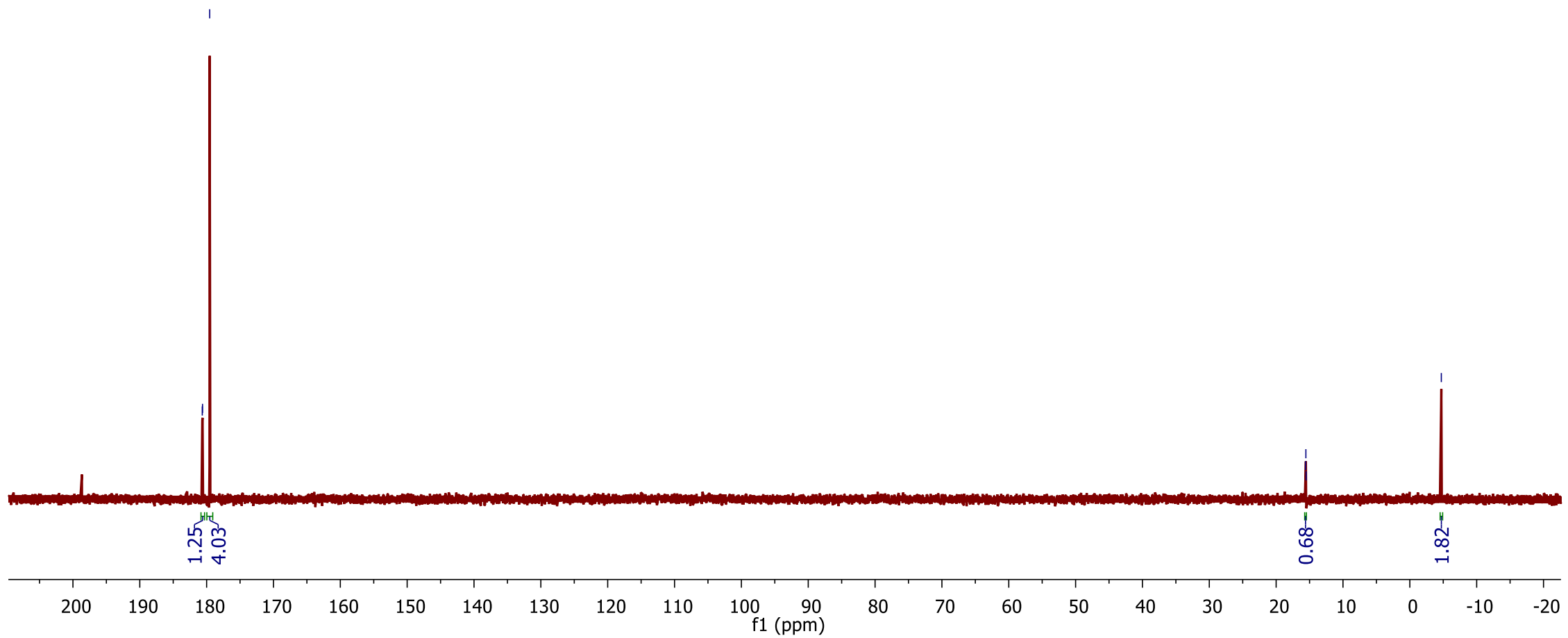

Figure S23. ${ }^{31} \mathrm{P}$ NMR of ( $\left.{ }^{\mathrm{tBu}} \mathrm{POCOP}\right) \operatorname{Ir}\left(\mathrm{PPh}_{3}\right)$ and $\left({ }^{\mathrm{tBu}} \mathrm{POCOP}\right) \operatorname{Ir}\left(\mathrm{NCCH}_{3}\right)$ equilibrium after $24 \mathrm{~h}$, using 8 equivalents of $\mathrm{MeCN}$. 


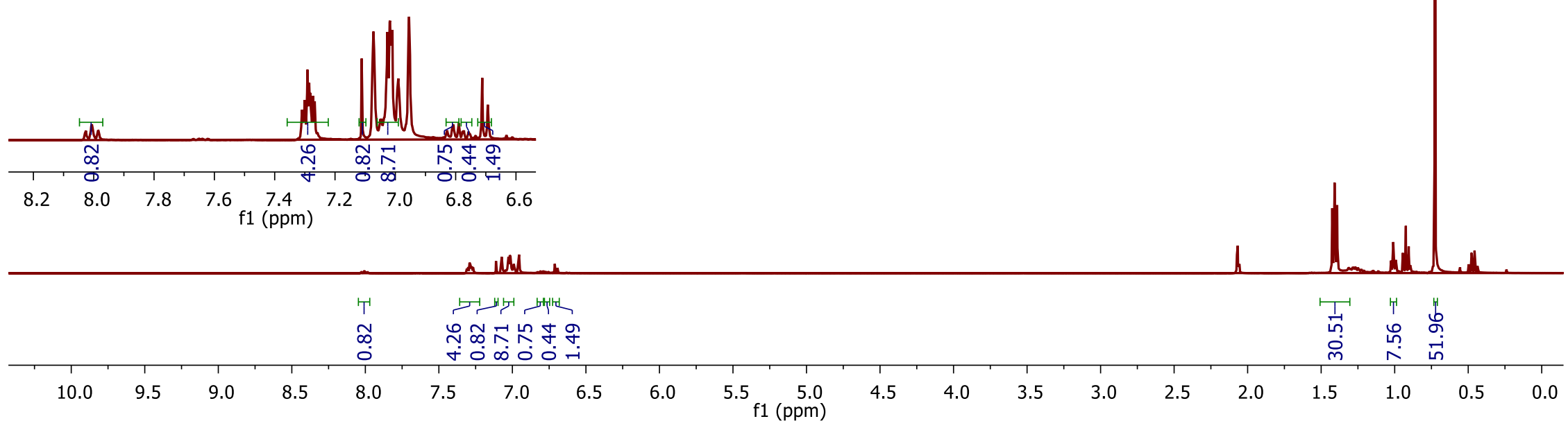

Figure S24. ${ }^{1} \mathrm{H}$ NMR of ( $\left.{ }^{\mathrm{BBu}} \mathrm{POCOP}\right) \operatorname{Ir}\left(\mathrm{PPh}_{3}\right)$ and ( $\left.{ }^{\mathrm{tBu}} \mathrm{POCOP}\right) \operatorname{Ir}\left(\mathrm{NCCH}_{3}\right)$ equilibrium after $24 \mathrm{~h}$, using 16 equivalents of $\mathrm{MeCN}$. 


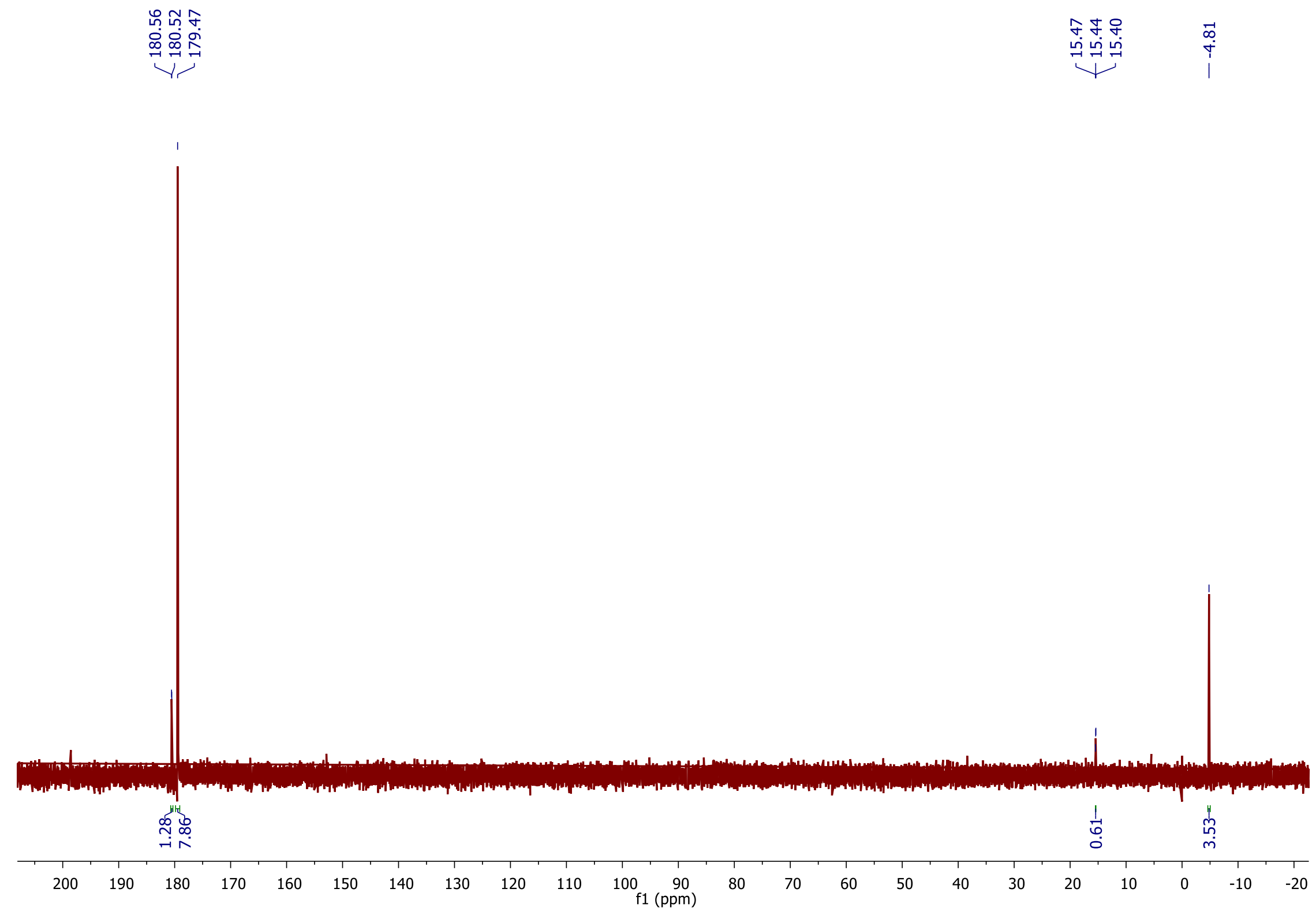

Figure S25. ${ }^{31} \mathrm{P}$ NMR of ( $\left.{ }^{\mathrm{tBu}} \mathrm{POCOP}\right) \operatorname{Ir}\left(\mathrm{PPh}_{3}\right)$ and $\left({ }^{\mathrm{tBu}} \mathrm{POCOP}\right) \operatorname{Ir}\left(\mathrm{NCCH}_{3}\right)$ equilibrium after $24 \mathrm{~h}$, using 16 equivalents of $\mathrm{MeCN}$. 
12. Representative ${ }^{1} \mathrm{H},{ }^{31} \mathrm{P}$ NMRs for addition of free phosphine to displacement of Ir-PPh ${ }_{3}$ and pyridine after equilibrium

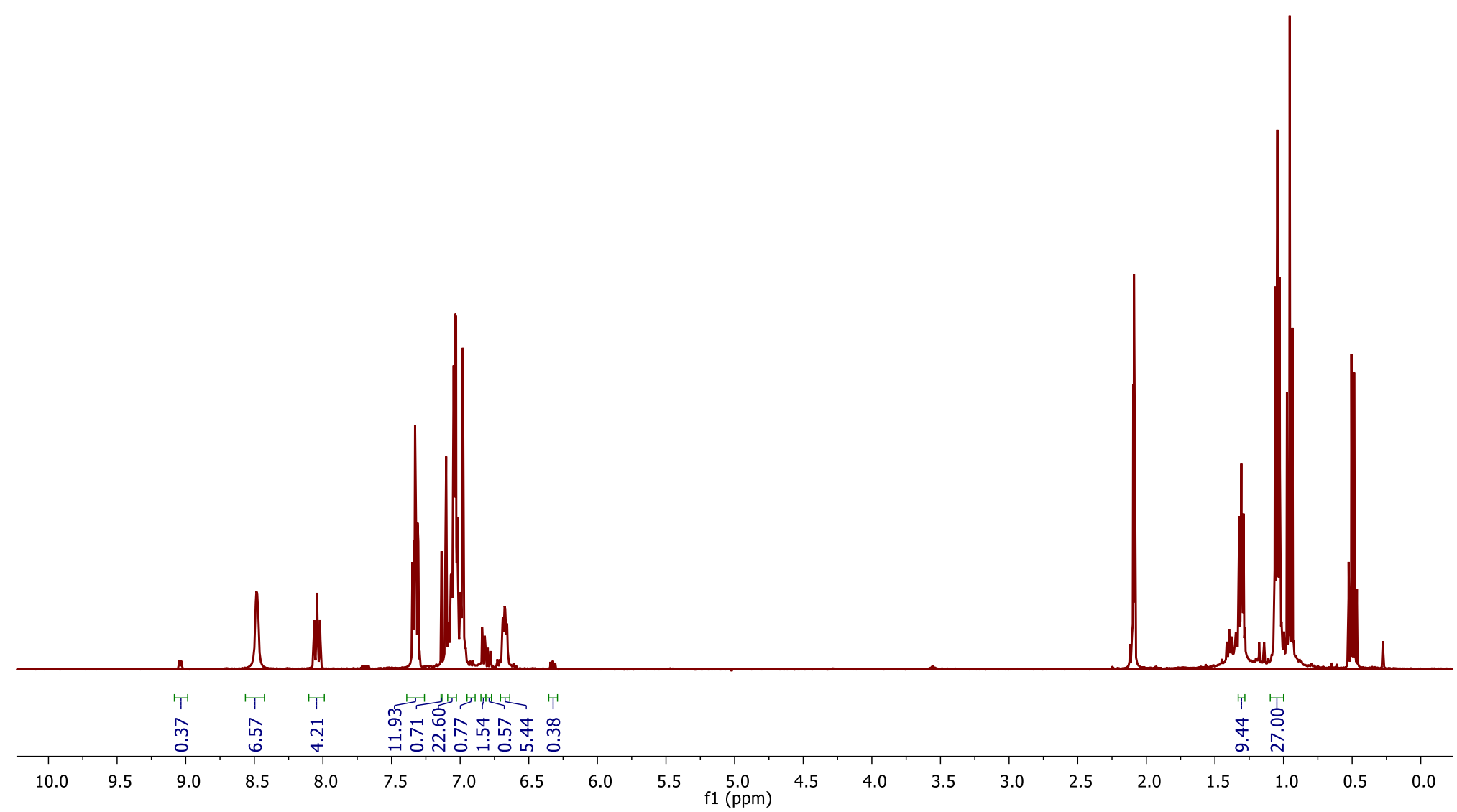

Figure S26. ${ }^{1} \mathrm{H}$ NMR of ( $\left.{ }^{\mathrm{B} u} \mathrm{POCOP}\right) \operatorname{Ir}\left(\mathrm{PPh}_{3}\right)$ and $\left({ }^{\mathrm{tBu}} \mathrm{POCOP}\right) \operatorname{Ir}(\mathrm{Py})$ equilibrium after $24 \mathrm{~h}$, using 2 equivalents of pyridine and 1 equivalent of $\mathrm{PPh}_{3}$. 


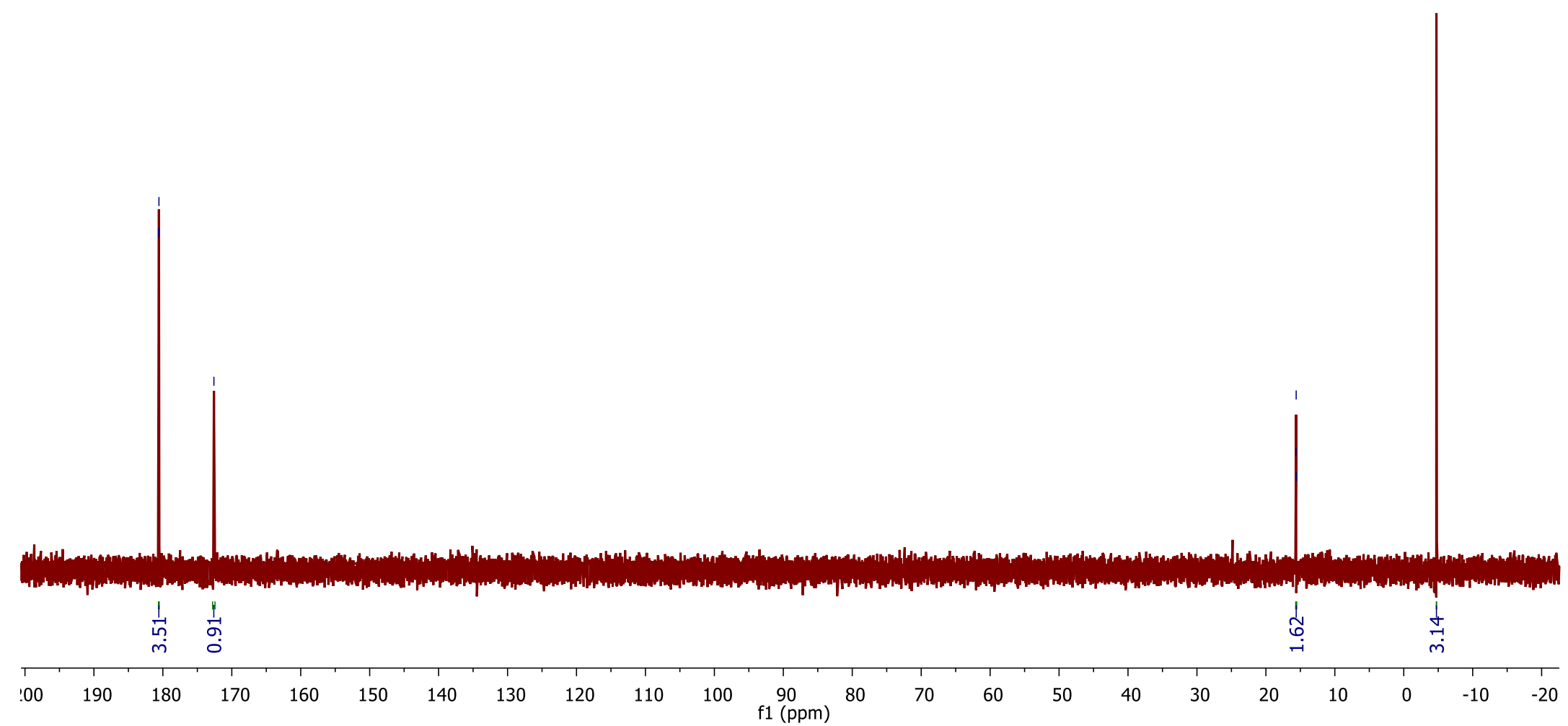

Figure S27. ${ }^{31} \mathrm{P}$ NMR of ( $\left.{ }^{\mathrm{B} u} \mathrm{POCOP}\right) \operatorname{Ir}\left(\mathrm{PPh}_{3}\right)$ and $\left({ }^{\mathrm{tBu}} \mathrm{POCOP}\right) \operatorname{Ir}(\mathrm{Py})$ equilibrium after $24 \mathrm{~h}$, using 2 equivalents of pyridine and 1 equivalent of $\mathrm{PPh}_{3}$. 


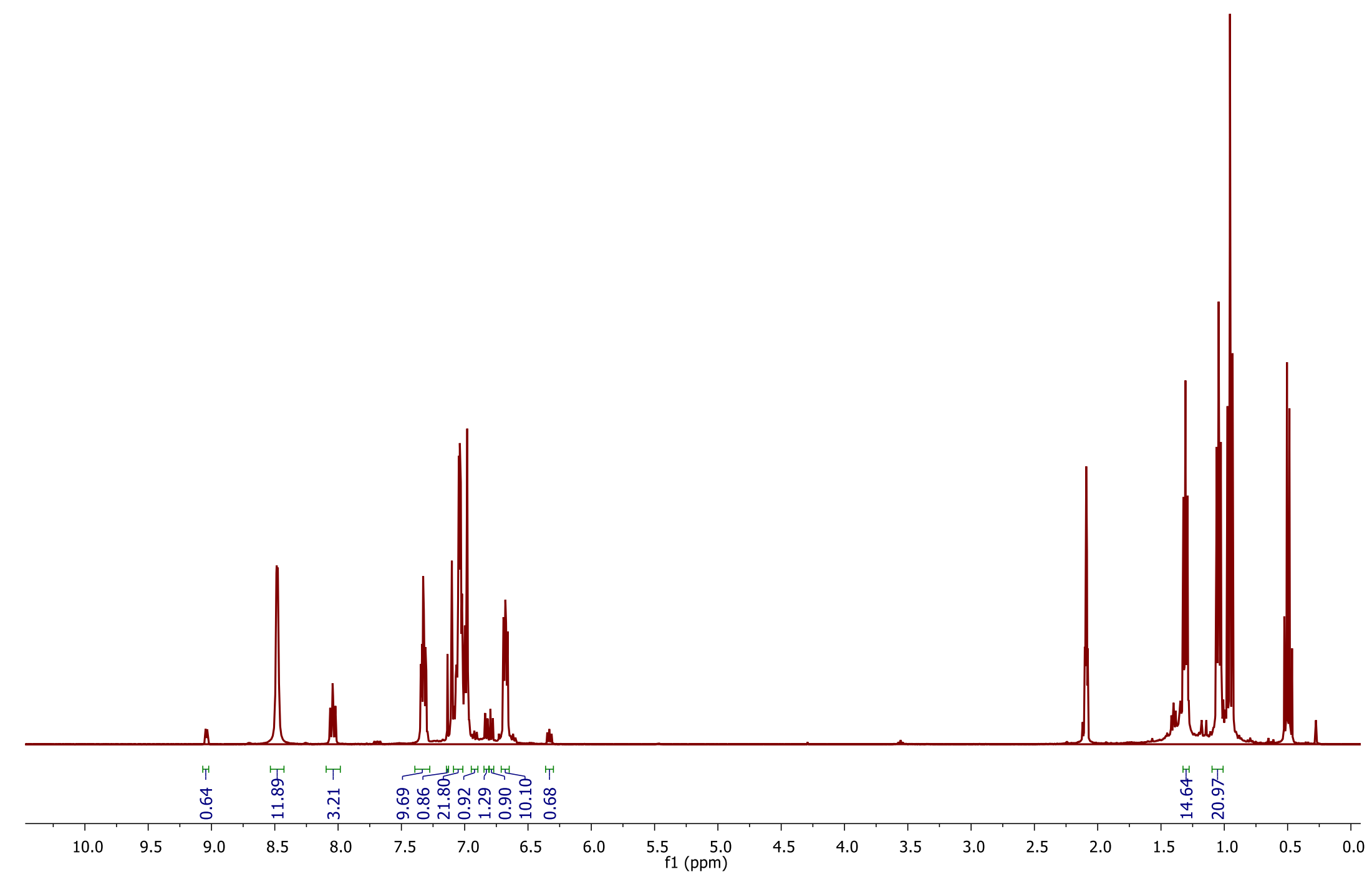

Figure S28. ${ }^{1} \mathrm{H}$ NMR of ( $\left.{ }^{\mathrm{tBu}} \mathrm{POCOP}\right) \operatorname{Ir}\left(\mathrm{PPh}_{3}\right)$ and $\left({ }^{\mathrm{tBu} P O C O P}\right) \operatorname{Ir}(\mathrm{Py})$ equilibrium after $24 \mathrm{~h}$, using 4 equivalents of pyridine and 1 equivalent of $\mathrm{PPh}_{3}$. 


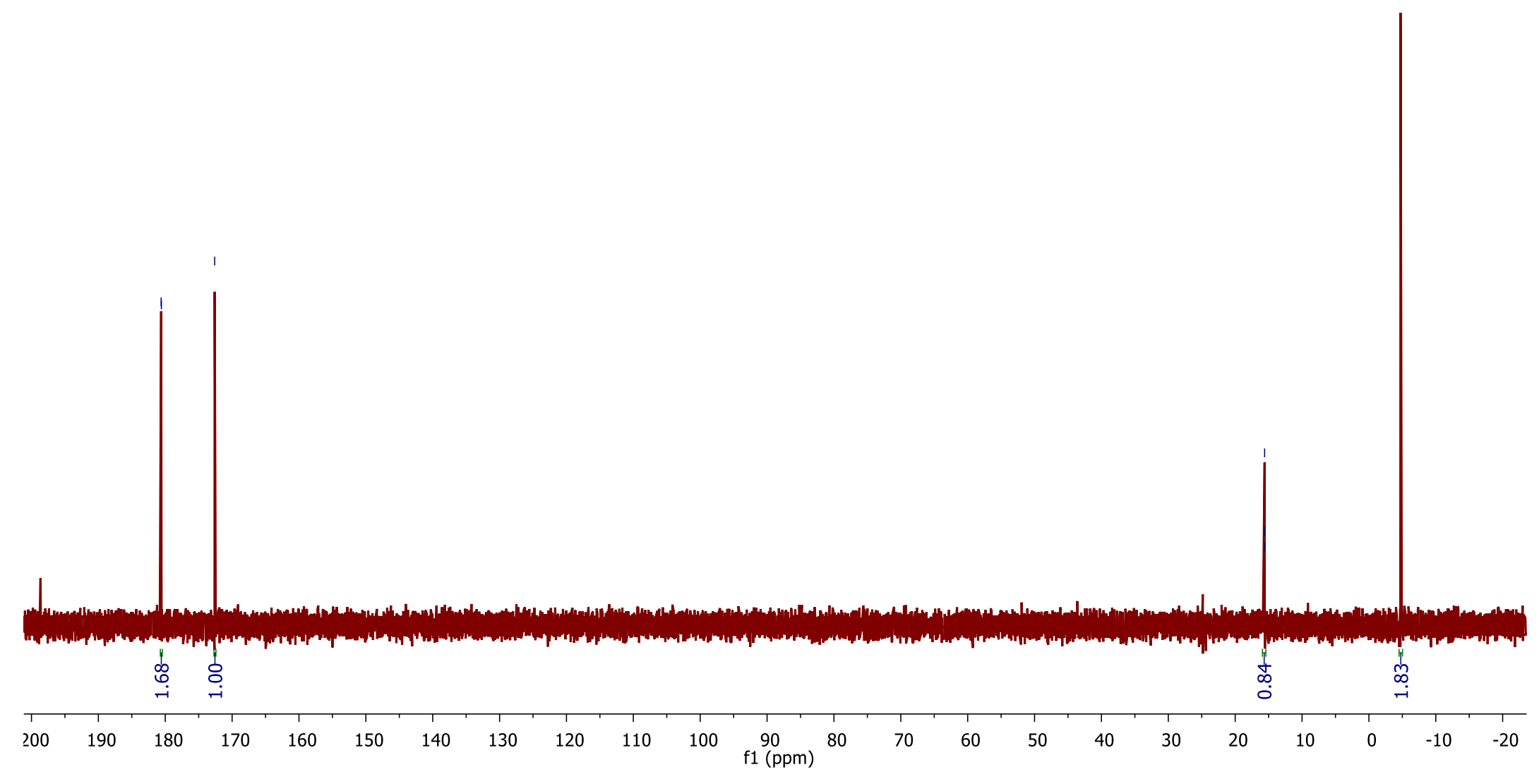

Figure S29. ${ }^{31} \mathrm{P}$ NMR of ( $\left.{ }^{\mathrm{BBu}} \mathrm{POCOP}\right) \operatorname{Ir}\left(\mathrm{PPh}_{3}\right)$ and $\left({ }^{\mathrm{tBu}} \mathrm{POCOP}\right) \operatorname{Ir}(\mathrm{Py})$ equilibrium after $24 \mathrm{~h}$, using 4 equivalents of pyridine and 1 equivalent of $\mathrm{PPh}_{3}$. 
13. ${ }^{1} \mathrm{H}$ NMR of $\left({ }^{\mathrm{tBu}} \mathrm{POCOP}\right) \operatorname{Ir}\left(\mathrm{PPh}_{3}\right)$ and excess 2,6 -lutidine.

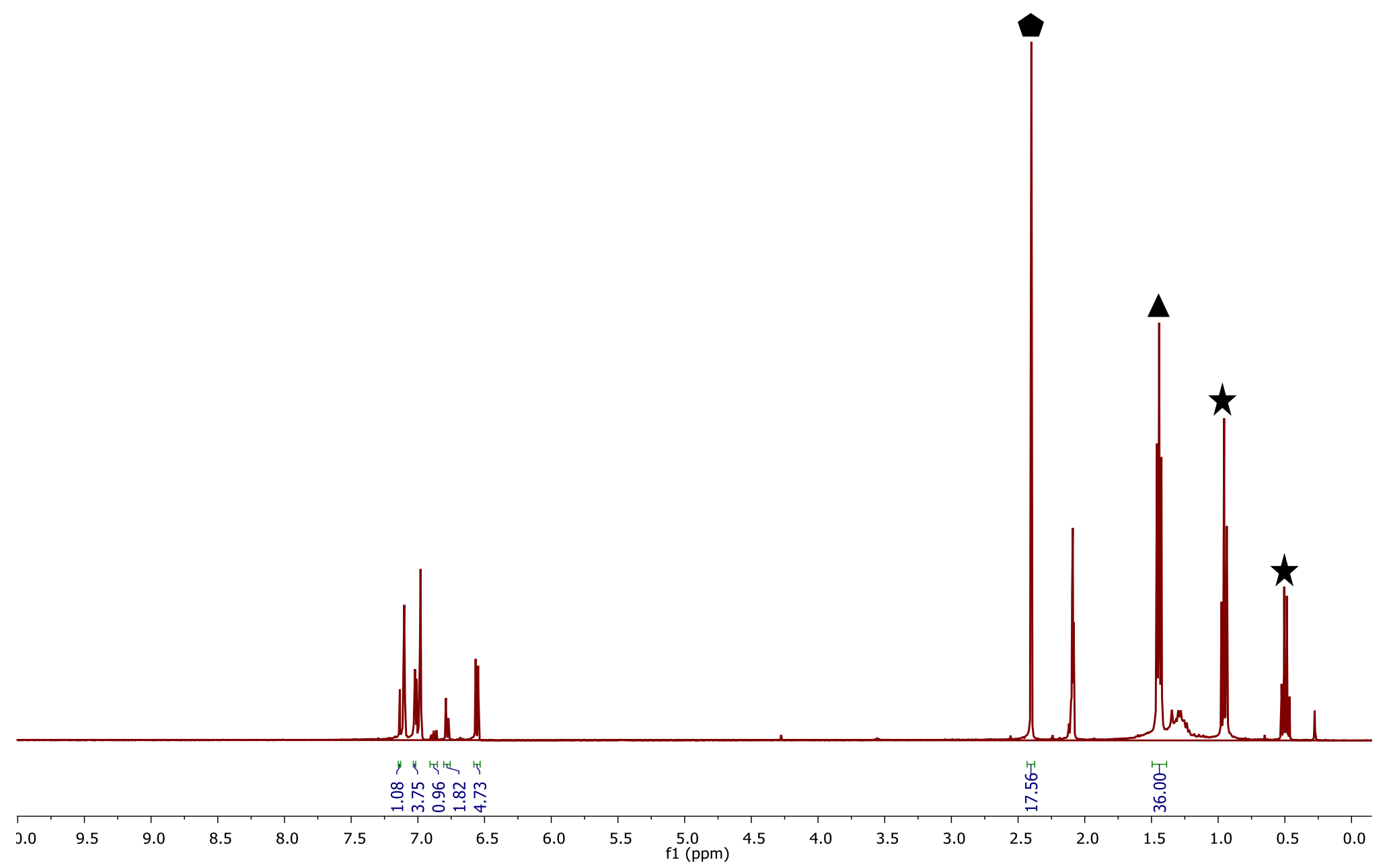

Figure S30. ${ }^{1} \mathrm{H}$ NMR of ( $\left.{ }^{\mathrm{tBu}} \mathrm{POCOP}\right) \operatorname{Ir}\left(\mathrm{PPh}_{3}\right)$ and excess 2,6-lutidine. and $(\boldsymbol{\hbar})$ represents internal standard. 
14. Representative ${ }^{1} \mathrm{H},{ }^{31} \mathrm{P}$ NMRs for displacement of pyridine and acetonitrile by $\mathrm{PPh}_{3}$ after equilibrium generation

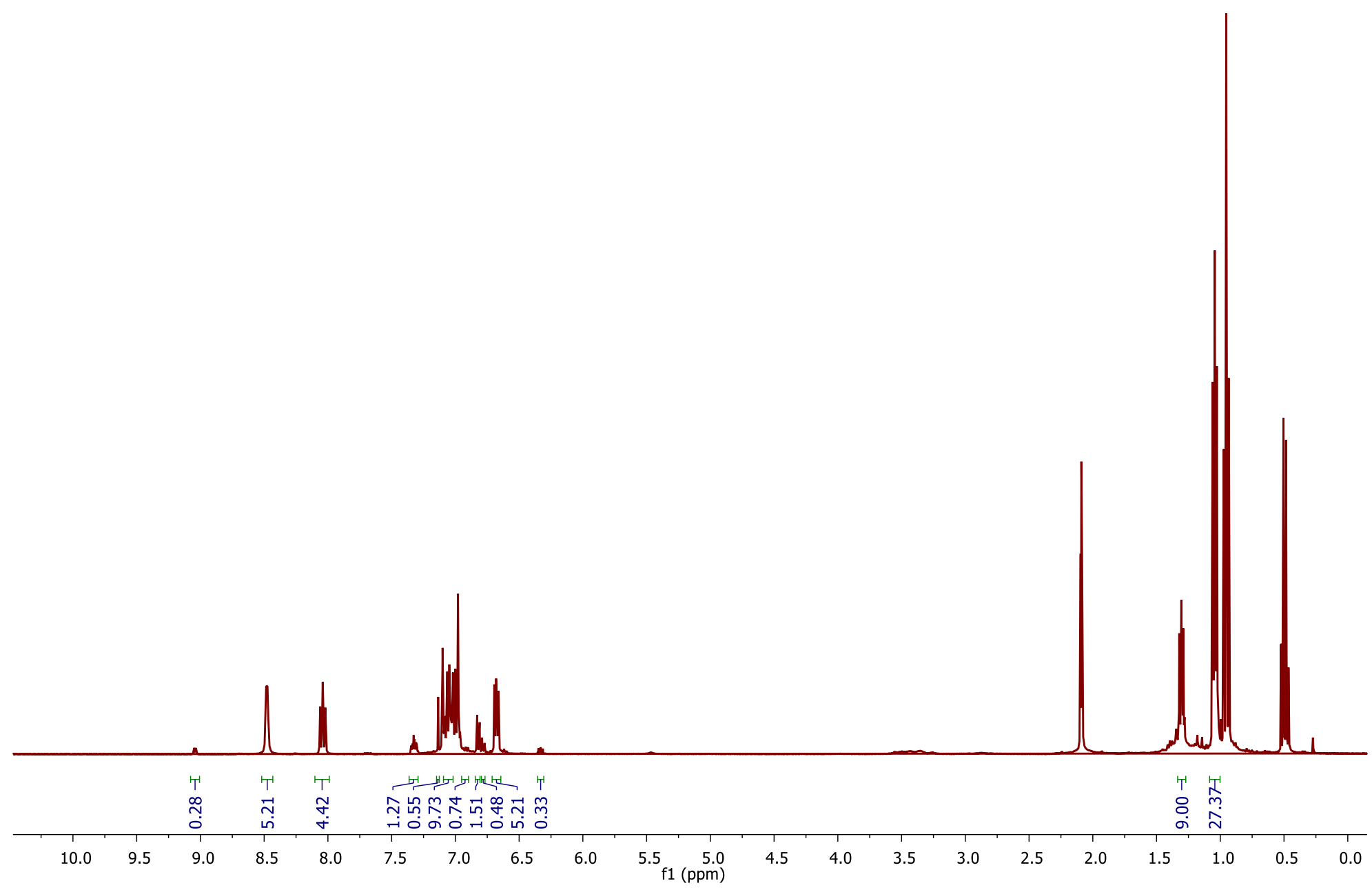

Figure S31. ${ }^{1} \mathrm{H}$ NMR of ( $\left.{ }^{\mathrm{tBu}} \mathrm{POCOP}\right) \operatorname{Ir}(\mathrm{Py})$ and ( $\left.{ }^{\mathrm{tBu}} \mathrm{POCOP}\right) \operatorname{Ir}\left(\mathrm{PPh}_{3}\right)$ equilibrium after $24 \mathrm{~h}$, using 1 equivalent of $\mathrm{PPh}_{3}$. 


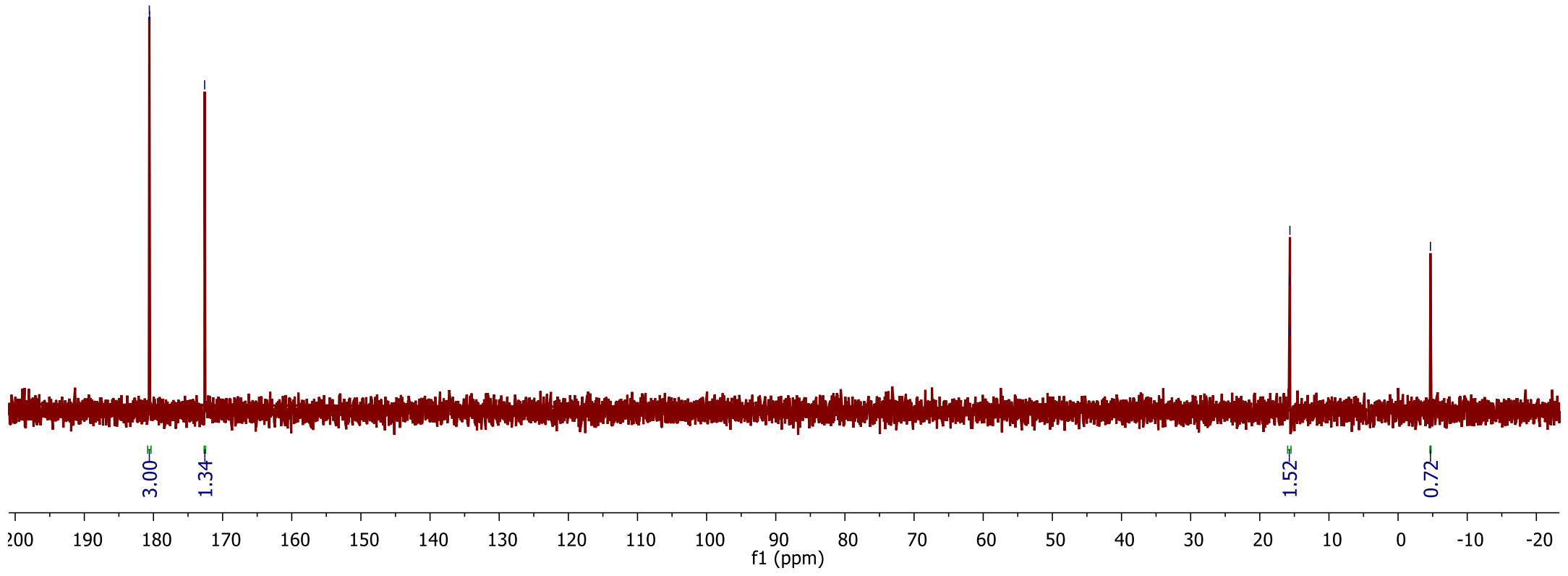

Figure S32. ${ }^{31} \mathrm{P}$ NMR of ( $\left.{ }^{\mathrm{tBu}} \mathrm{POCOP}\right) \operatorname{Ir}(\mathrm{Py})$ and $\left({ }^{\mathrm{tBu}} \mathrm{POCOP}\right) \operatorname{Ir}\left(\mathrm{PPh}_{3}\right)$ equilibrium after $24 \mathrm{~h}$, using 1 equivalent of $\mathrm{PPh}_{3}$. 


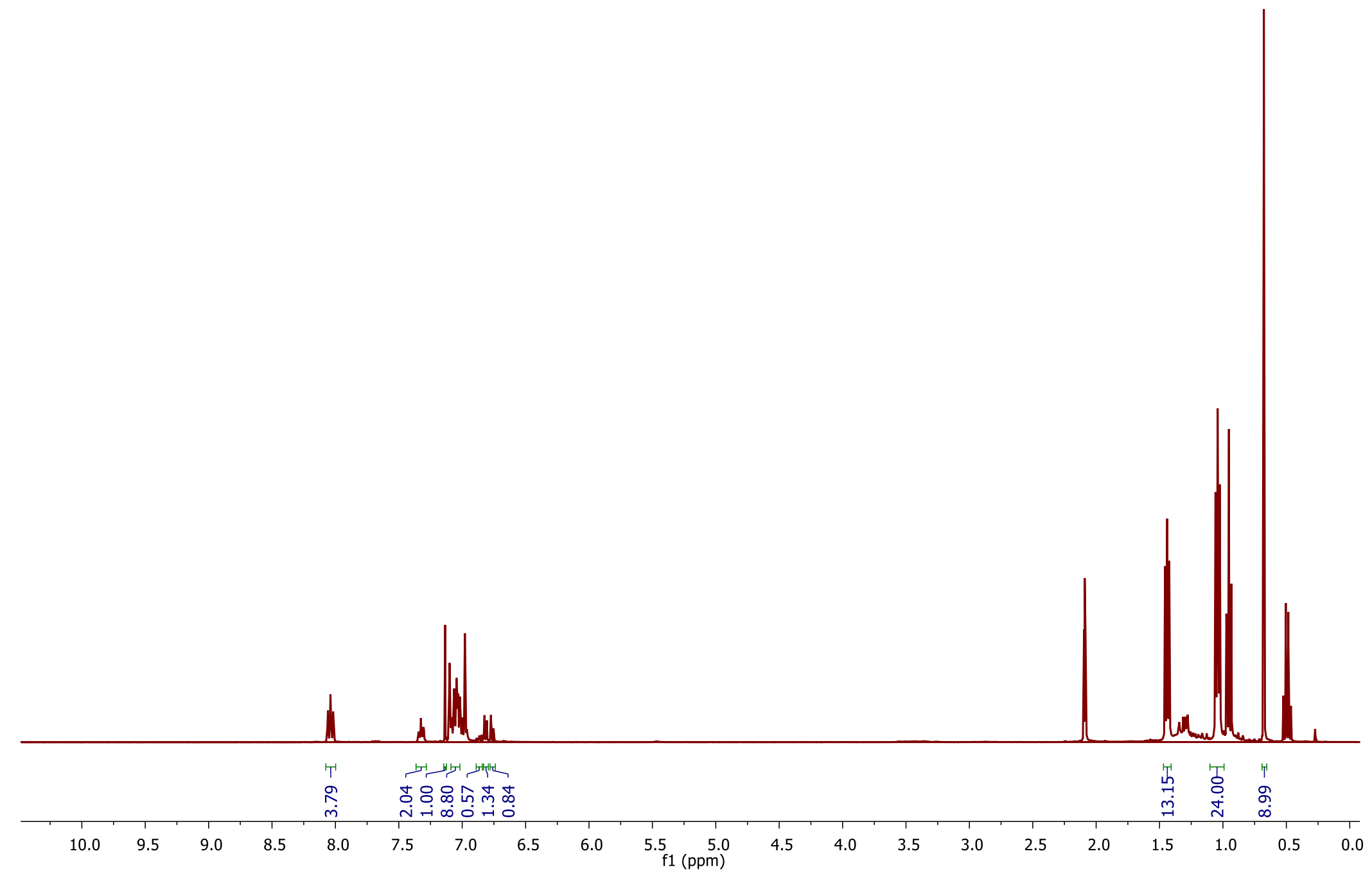

Figure S33. ${ }^{1} \mathrm{H}$ NMR of ( $\left.{ }^{\mathrm{tBu}} \mathrm{POCOP}\right) \operatorname{Ir}\left(\mathrm{NCCH}_{3}\right)$ and $\left({ }^{\mathrm{tBu}} \mathrm{POCOP}\right) \operatorname{Ir}\left(\mathrm{PPh}_{3}\right)$ equilibrium after $24 \mathrm{~h}$, using 1 equivalent of $\mathrm{PPh}_{3}$ 


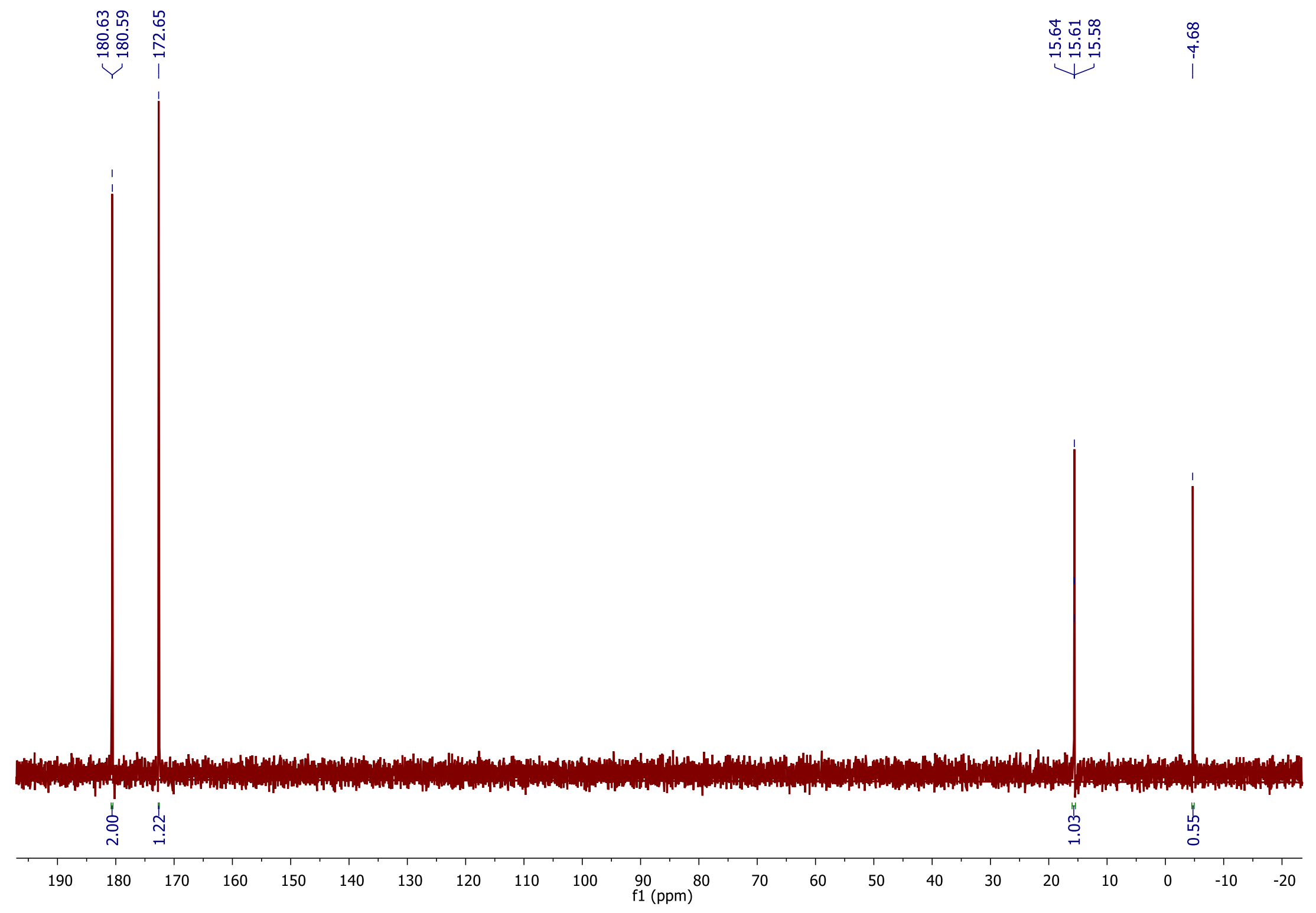

Figure S34. ${ }^{31} \mathrm{P}$ NMR of ( $\left.{ }^{\mathrm{tBu}} \mathrm{POCOP}\right) \operatorname{Ir}\left(\mathrm{NCCH}_{3}\right)$ and $\left({ }^{\mathrm{BBu}} \mathrm{POCOP}\right) \operatorname{Ir}\left(\mathrm{PPh}_{3}\right)$ equilibrium after $24 \mathrm{~h}$, using 1 equivalent of $\mathrm{PPh}$. 


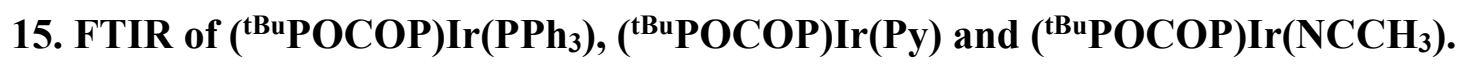

( $\left.{ }^{\mathrm{tBu} P O C O P}\right) \operatorname{Ir}\left(\mathrm{PPh}_{3}\right)$ FTIR $\left(\mathrm{cm}^{-1}\right): 2940.91,2886.64,2856.06,1565.74,1476.16,1436.96,1362.59,1385.80,1264.68,1226.44$, $1087.45,1030.22,843.84,808.10,767.32,751.49,725.53,707.83,693.36,625.23,571.07,530.21,513.25,470.88$.

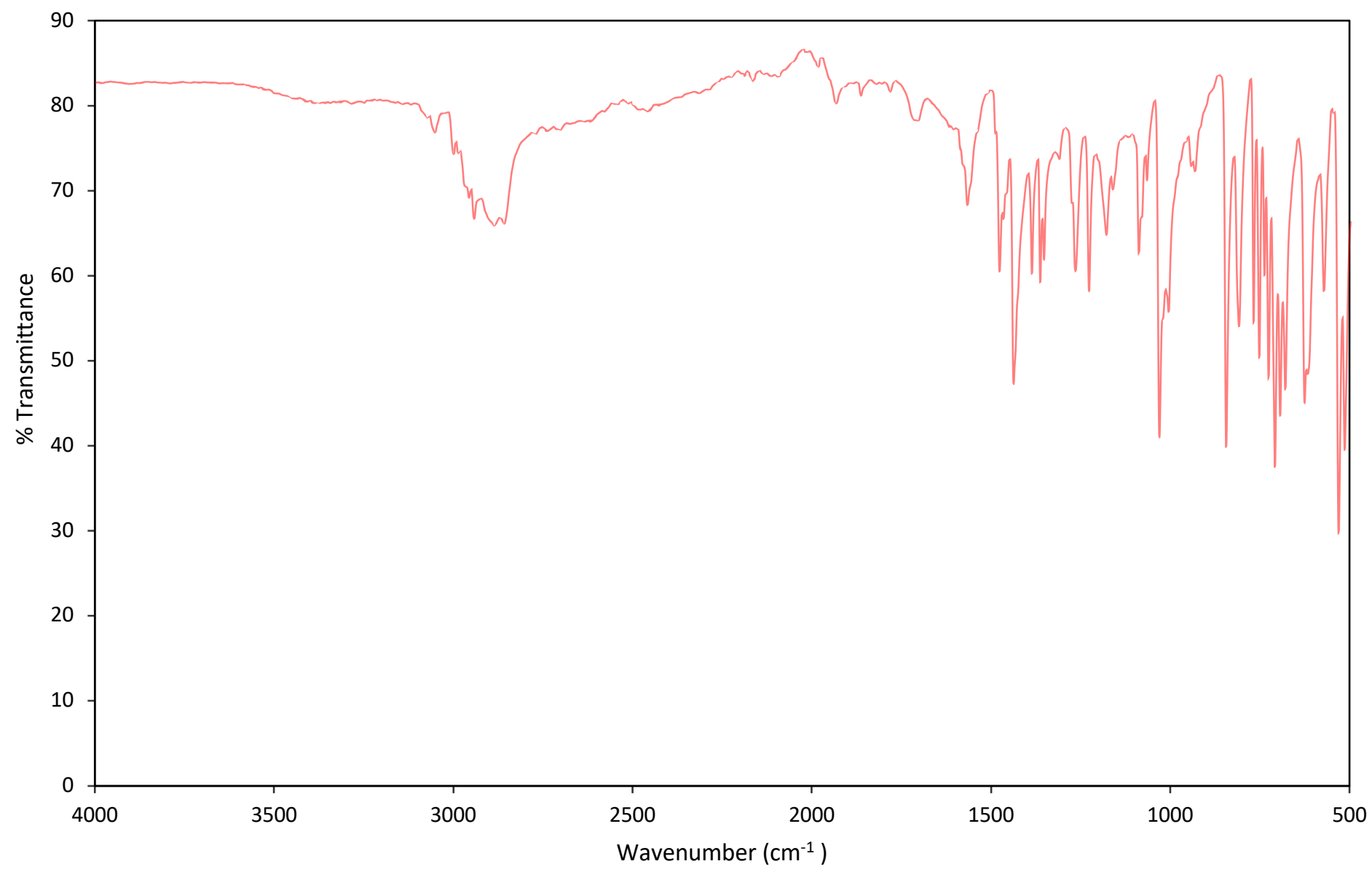

Figure S35. IR spectrum of ( $\left.{ }^{\mathrm{tBu}} \mathrm{POCOP}\right) \operatorname{Ir}\left(\mathrm{PPh}_{3}\right)$. 
('BupOCOP)Ir(Py) FTIR ( $\left.\mathrm{cm}^{-1}\right)$ : 2932.23， 2934.34， 2889.80 2859.43，1546.64, 1466.93, 1435.34, 1409.86, 1383.37, 1363.40, $1239.48,1199.88,1022.53,989.43,823.83,808.85,759.52,733.89,672.72,623.70,576.29,486.42,450.74$. CHN results Anal. calc.: C, 48.49; H, 6.63; N, 2.09; Found C, 49.48; H, 7.13; N, 2.61 .

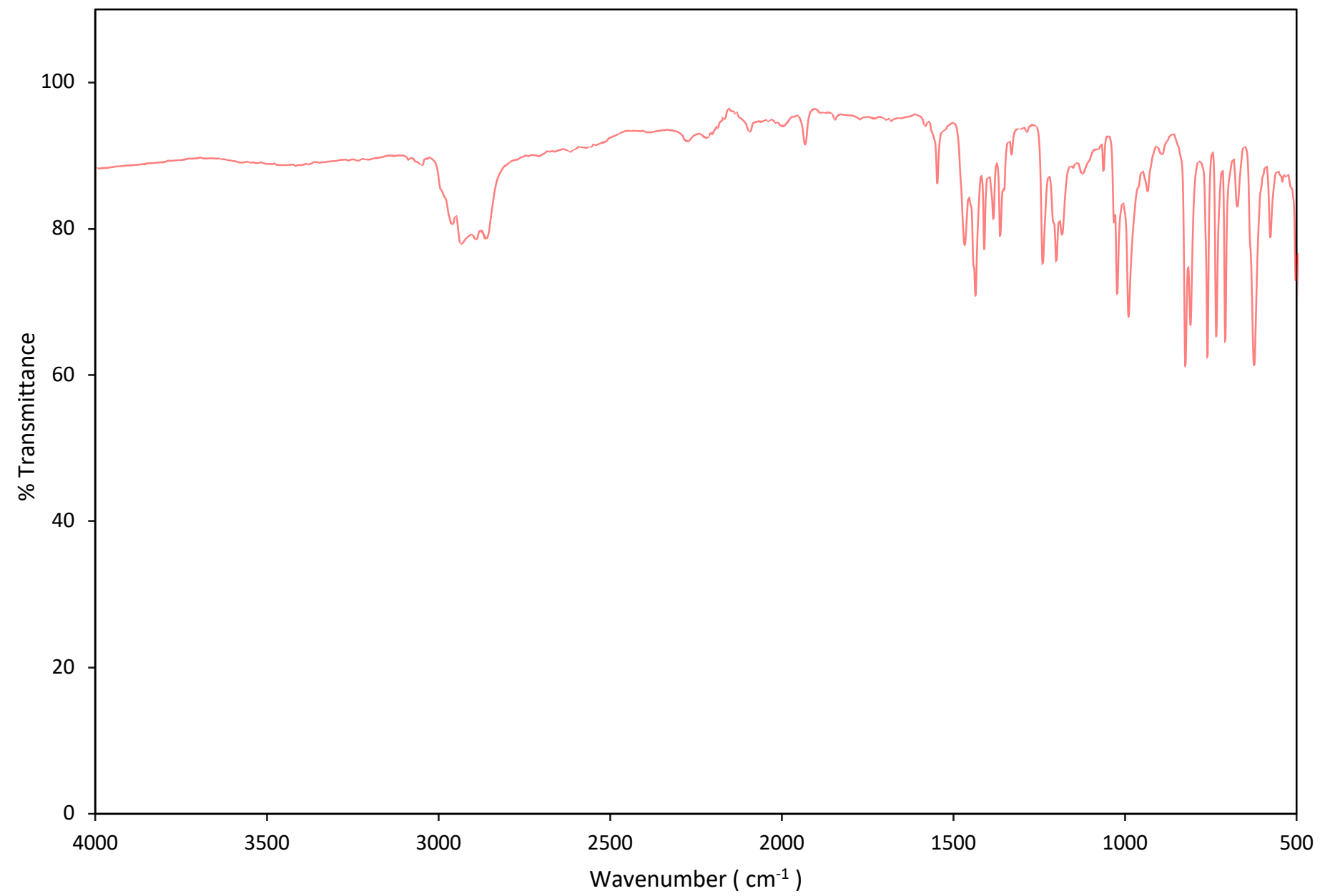


Figure S36. IR spectrum of ( $\left.{ }^{\mathrm{B} u} \mathrm{POCOP}\right) \operatorname{Ir}(\mathrm{Py})$.

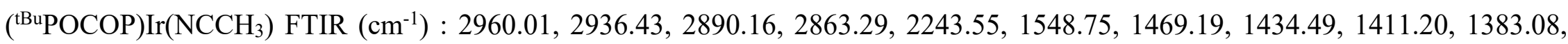
$1361.52,1228.67,1203.12,1183.94,1021.72,984.29,825.67,808.29,757.18,736.30,675.61,625.53,576.85,486.65$.

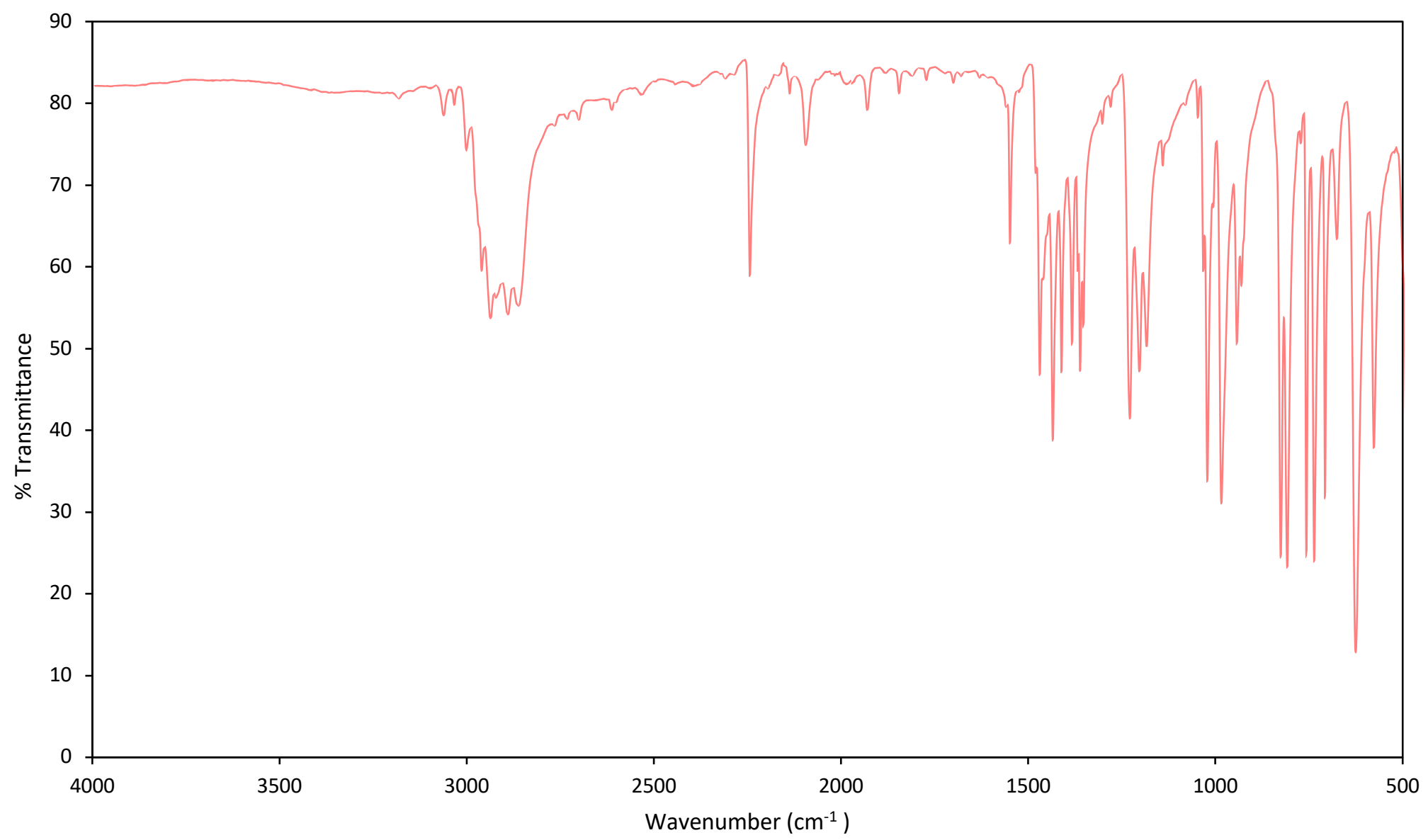

Figure S37. IR spectrum of ( $\left.{ }^{\mathrm{tBu} P O C O P}\right) \operatorname{Ir}(\mathrm{NCCH} 3)$. 


\section{UV-Vis Kinetic Data and Calculations}

Data Collection:

UV-vis spectra were collected on Silver-Nova Super Range TEC spectrometer, with SL1 Tungsten Halogen Lamp (visible and near-IR region) as the light source. The spectrometer was equipped with a CUV-TEMP cuvette holder (qpod 2e) with a path length of $1.0 \mathrm{~cm}$. The spectra were collected at three different temperature $\left(65^{\circ} \mathrm{C}, 75^{\circ} \mathrm{C}, 85^{\circ} \mathrm{C}\right)$ with a stir rate of $1200 \mathrm{rpm}$.

Before each kinetic run, a dark spectrum and a blank spectrum (of toluene) were collected. Inside

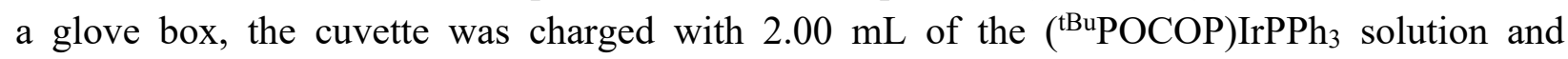
appropriate amounts of pyridine or acetonitrile stock solutions to achieve the desired concentrations. The cuvettes had a Chemglass stopper with an o-ring to ensure air-free conditions over the course of the experiments. The ( $\left.{ }^{\mathrm{tBu}} \mathrm{POCOP}\right) \mathrm{IrPPh}_{3}$ stock solutions were prepared at concentrations of $0.020 \mathrm{M}$ or $0.004225 \mathrm{M}$ for the pyridine or acetonitrile experiments, respectively. The stock solutions of Pyridine and Acetonitrile were prepared in toluene at a concentration of $5.00 \mathrm{M}$. For the kinetic run, each time cuvette was charged with $25 \mu \mathrm{L}$ of ( $\left.{ }^{\mathrm{tBu}} \mathrm{POCOP}\right) \mathrm{IrPPh}_{3}$ in 2 $\mathrm{ml}$ Toluene followed by $10.00 \mu \mathrm{L}$ (100 eq), $20.00 \mu \mathrm{L}$ (200 eq), $30.00 \mu \mathrm{L}$ (300 eq) of pyridine and acetonitrile stock solution.

Table S5. The forward rate constants $\left(\mathrm{k}_{1}\right)$ for pyridine and acetonitrile at different temperatures calculated using Equation2.

\begin{tabular}{|l|l|l|l|l|l|}
\hline S.no & Ligand & $\begin{array}{l}\text { Temperature } \\
\left({ }^{\circ} \mathrm{C}\right)\end{array}$ & $100 \mathrm{eq}$ & $200 \mathrm{eq}$ & $300 \mathrm{eq}$ \\
\hline 1 & Pyridine & 65 & $\begin{array}{l}1.61 \mathrm{E}- \\
03\end{array}$ & $5.13 \mathrm{E}-04$ & $\begin{array}{l}3.52 \mathrm{E}- \\
04\end{array}$ \\
\hline 2 & Pyridine & 75 & $\begin{array}{l}3.56 \mathrm{E}- \\
03\end{array}$ & $2.81 \mathrm{E}-03$ & $\begin{array}{l}1.50 \mathrm{E}- \\
03\end{array}$ \\
\hline 3 & Pyridine & 85 & $\begin{array}{l}2.32 \mathrm{E}- \\
03\end{array}$ & $9.21 \mathrm{E}-03$ & $\begin{array}{l}5.40 \mathrm{E}- \\
03\end{array}$ \\
\hline 4 & Acetonitrile & 65 & $\begin{array}{l}3.43 \mathrm{E}- \\
03\end{array}$ & $1.29 \mathrm{E}-03$ & $\begin{array}{l}7.38 \mathrm{E}- \\
04\end{array}$ \\
\hline 5 & Acetonitrile & 75 & $\begin{array}{l}8.91 \mathrm{E}- \\
03\end{array}$ & $4.38 \mathrm{E}-03$ & $\begin{array}{l}2.16 \mathrm{E}- \\
03\end{array}$ \\
\hline 6 & Acetonitrile & 85 & $\begin{array}{l}2.94 \mathrm{E}- \\
02\end{array}$ & $1.37 \mathrm{E}-02$ & $\begin{array}{l}9.89 \mathrm{E}- \\
03\end{array}$ \\
& & & & & \\
\hline
\end{tabular}


Molar Absorptivity Calculation for a) ( $\left.{ }^{\mathrm{BBu}} \mathrm{POCOP}\right) \operatorname{Ir}(\mathrm{Py})$, b) ( $\left.{ }^{\mathrm{tBu}} \mathrm{POCOP}\right) \operatorname{Ir}\left(\mathrm{NCCH}_{3}\right)$, c) ( $\left.{ }^{\mathrm{tBu}} \mathrm{POCOP}\right) \operatorname{Ir}\left(\mathrm{PPh}_{3}\right)$.
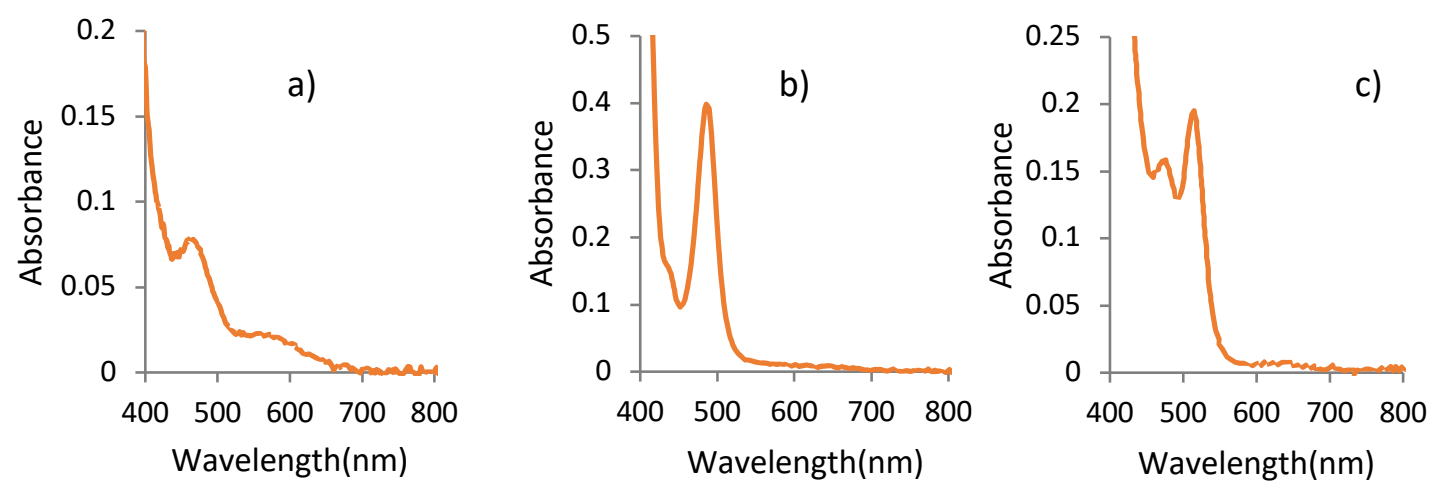

Figure S38. Plot of Absorbance Vs Wavelength for a) ( $\left.\left.{ }^{\mathrm{tBu}} \mathrm{POCOP}\right) \operatorname{Ir}(\mathrm{Py}), \mathrm{b}\right)$ ('BuPOCOP) $\operatorname{Ir}\left(\mathrm{NCCH}_{3}\right)$, c) ( $\left.{ }^{\mathrm{BBu}} \mathrm{POCOP}\right) \operatorname{Ir}\left(\mathrm{PPh}_{3}\right)$.

\section{Data Fitting}

The intensity data at $515.505 \mathrm{~nm}$ was extracted and plotted as function of time. Kinetic rate laws were used to fit the intensity data by adjusting the value of the rate. For the fit, we choose the time frame where continuous smooth decrease was observed. The employed models are discussed below and correspond to the following equilibrium:

$$
\mathrm{ML}+\mathrm{L}^{\prime} \rightleftharpoons \mathrm{ML}^{\prime}+\mathrm{L}
$$

1. Reversible reaction with $1^{\text {st }}$ order dependence on all species (associative mechanism): The reversible bimolecular transformation above could have the following rate law where $\mathrm{k}_{1}$ and $\mathrm{k}_{2}$ are the forward and backward rate constants, respectively. The value $\mathrm{x}(\mathrm{t})$ represents the change as a function of time.

$$
\frac{d x(t)}{d t}=k_{1}\left[C_{M L}-x(t)\right]\left[C_{L^{\prime}}-x(t)\right]-k_{2}\left[C_{M L^{\prime}}+x(t)\right]\left[C_{L}+x(t)\right]
$$

The integrated rate law was determined using the Maple software package and it was simplified further by symbols:

$$
x(t)=2 \tan (\lambda t / 2)\left[\frac{\delta}{\lambda+(\gamma \cdot \tan (\gamma t / 2))}\right]
$$

where $\beta=\mathrm{k}_{1}-\mathrm{k}_{2} ; \delta=\mathrm{k}_{1} \mathrm{C}_{\mathrm{ML}} \mathrm{C}_{\mathrm{L}^{\prime}}-\mathrm{k}_{2} \mathrm{C}_{\mathrm{ML}^{\prime}} \mathrm{C}_{\mathrm{L}} ; \gamma=\mathrm{k}_{1}\left(\mathrm{C}_{\mathrm{ML}}+\mathrm{C}_{\mathrm{L}^{\prime}}\right)+\mathrm{k}_{2}\left(\mathrm{C}_{\mathrm{ML}^{\prime}}+\mathrm{C}_{\mathrm{L}}\right)$ and $\lambda=\sqrt{ } 4 \beta \delta-\gamma^{2}$ 
2. Reversible reaction with $1^{\text {st }}$ order dependence on metal complexes only (dissociative mechanism): In this reaction we assume that forward and reverse reaction have no dependence on the concentration of exogenous ligand. The differential equation expressing this model is as follow:

$$
\frac{d x(t)}{d t}=k_{1}\left[C_{M L}-x(t)\right]-k_{2}\left[C_{M L^{\prime}}+x(t)\right]
$$

The following integrated rate law was determined using MathCAD:

$$
x(t)=\left[\frac{\left(k_{1} C_{M L}+k_{2} C_{M L^{\prime}}\right)}{k_{1}+k_{2}}\right]\left[1-e^{-\left(k_{1}+k_{2}\right)}\right]
$$

\section{Determination of Rate Constants}

Absorbance $=\varepsilon C l$,

where $\varepsilon$ is molar absorptivity, $\mathrm{C}$ is concentration and 1 is path length. In this, UV- vis path length is $1 \mathrm{~cm}$.

Absorbance $_{\text {obs }}=\varepsilon_{\mathrm{ML}}[\mathrm{ML}]+\varepsilon_{\mathrm{ML}}\left[\mathrm{ML}^{\prime}\right]$

$\varepsilon_{\mathrm{ML}}$ is the molar absorptivity of the ( $\left.{ }^{\mathrm{tBu}} \mathrm{POCOP}\right) \operatorname{Ir}\left(\mathrm{PPh}_{3}\right)$

$\varepsilon_{\mathrm{ML}}$ is the molar absorptivity of ( $\left.{ }^{\mathrm{tBu}} \mathrm{POCOP}\right) \operatorname{Ir}(\mathrm{L})$ where $\mathrm{L}$ is pyridine or acetonitrile)

\section{Activation Parameters}

The enthalpy of activation and the entropy of activation were determined by evaluating the rate constants at different temperatures and plotting the data according to the Eyring equation:

$$
\ln \left(\frac{k}{T}\right)=-\frac{\Delta H^{\ddagger}}{R T}+\ln \frac{k^{\prime}}{h}+\frac{\Delta S^{\ddagger}}{R}
$$

$\mathrm{k}$ is rate constant, $\mathrm{T}$ is temperature in $\mathrm{K}$,

$\mathrm{R}$ is molar gas constant $\left(8.314 \mathrm{~J} \mathrm{~K}^{-1} \mathrm{~mol}^{-1}\right)$,

$\mathrm{k}^{\prime}$ is Boltzmann Constant $\left(1.381 * 10^{-23} \mathrm{~J} \mathrm{~K}^{-1}\right)$,

$\mathrm{h}$ is planck constant $\left(6.626^{*} 10^{-34} \mathrm{~J} \mathrm{~s}\right)$.

The Gibbs energy of activation was obtained using $\Delta G^{\ddagger}=\Delta H^{\ddagger}-T \Delta S^{\ddagger}$ 

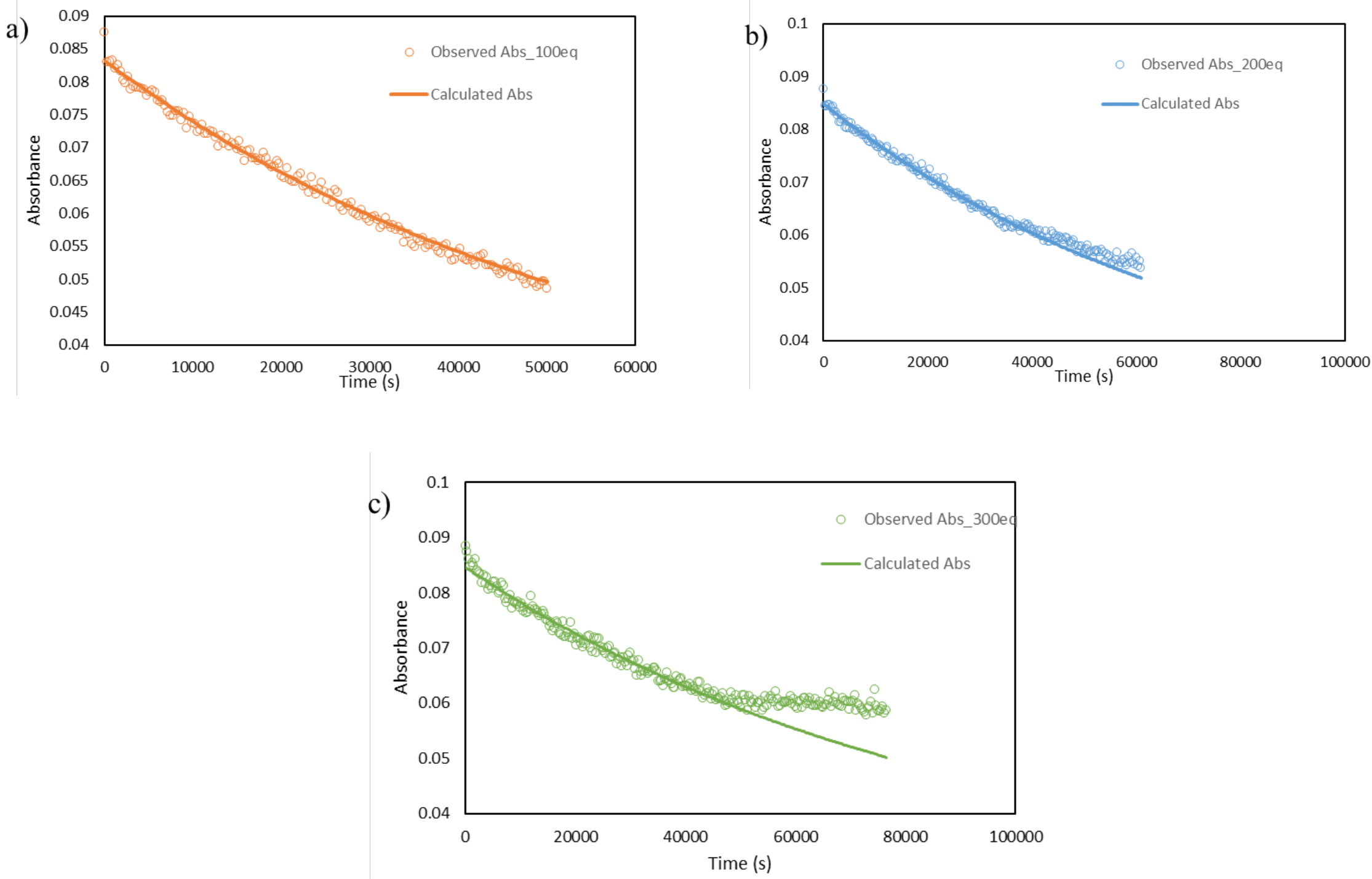

Figure S39. Kinetic run plot for a) 100 eq, b) 200 eq, c) 300 eq of Acetonitrile at $65^{\circ} \mathrm{C}$. 

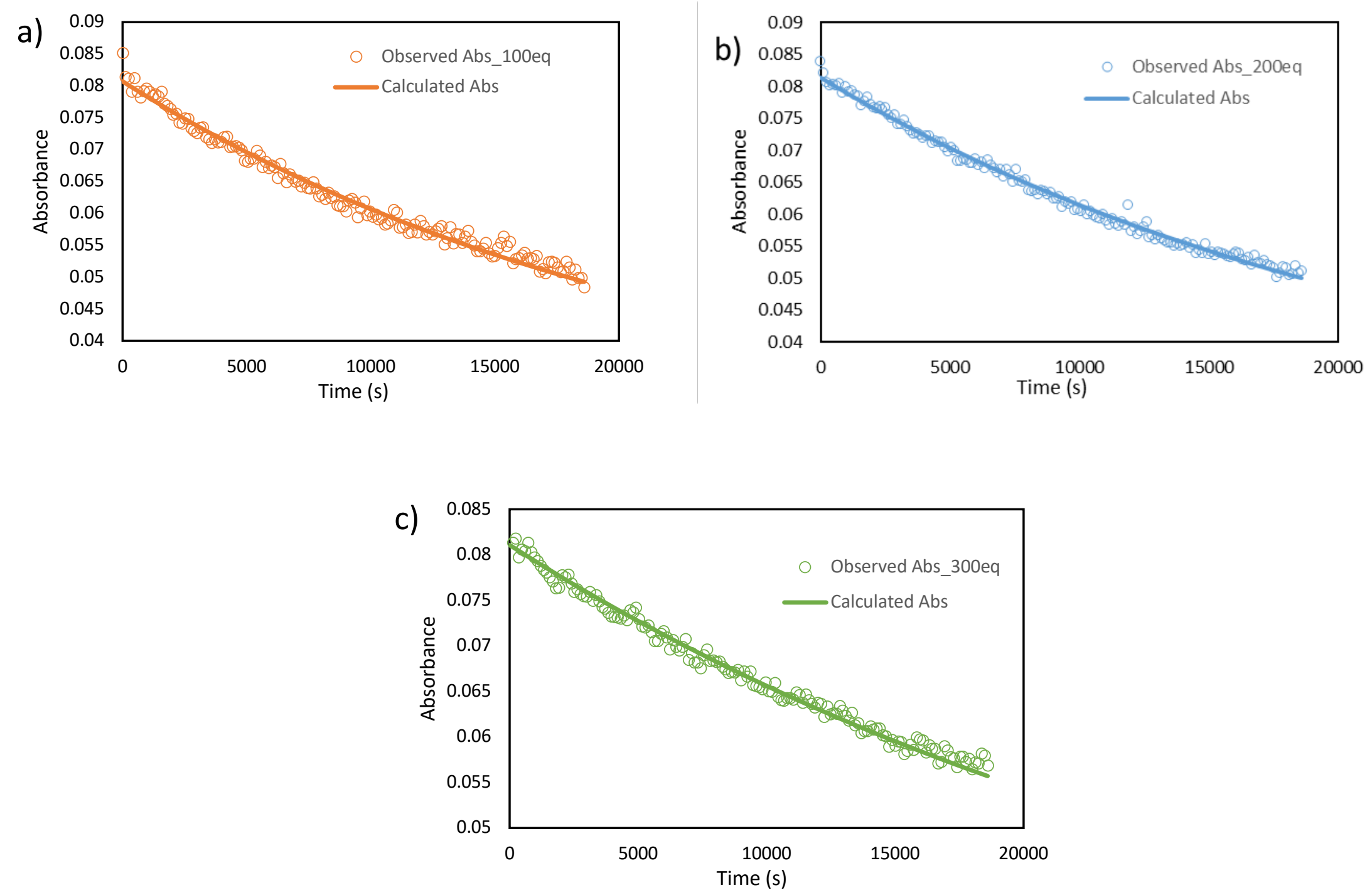

Figure S40. Kinetic run plot for a) 100 eq, b) 200 eq, c) 300 eq of Acetonitrile at $75{ }^{\circ} \mathrm{C}$. 
a)

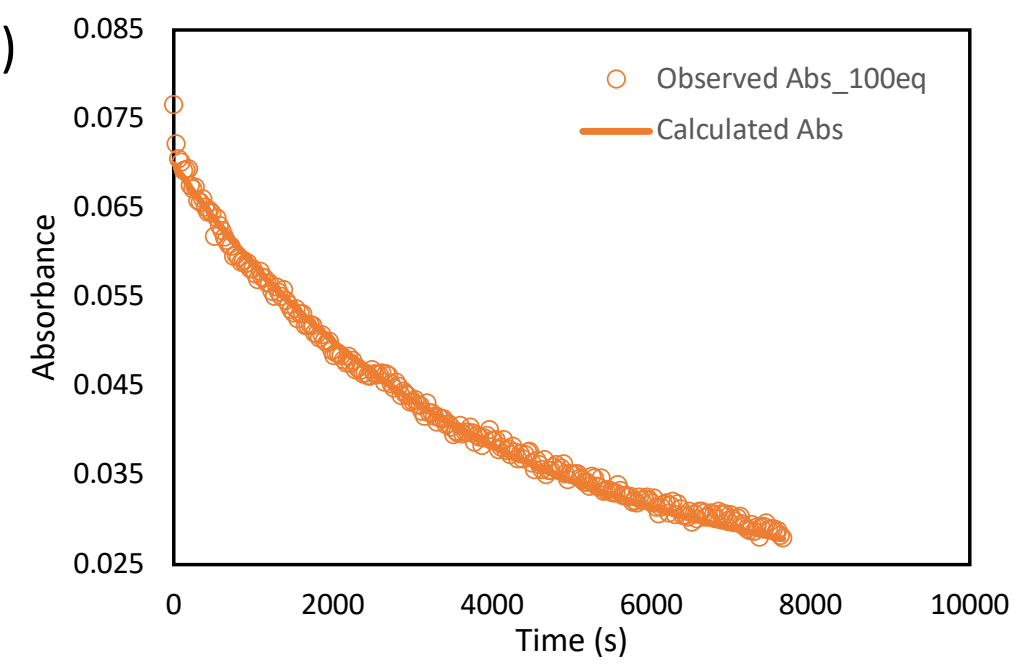

b)

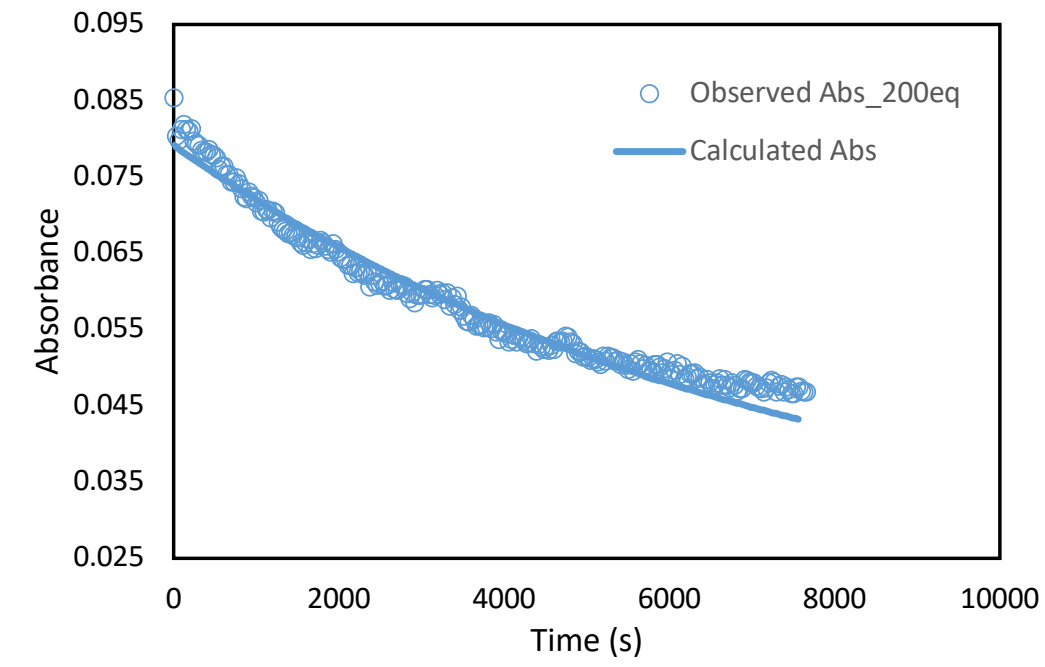

c)

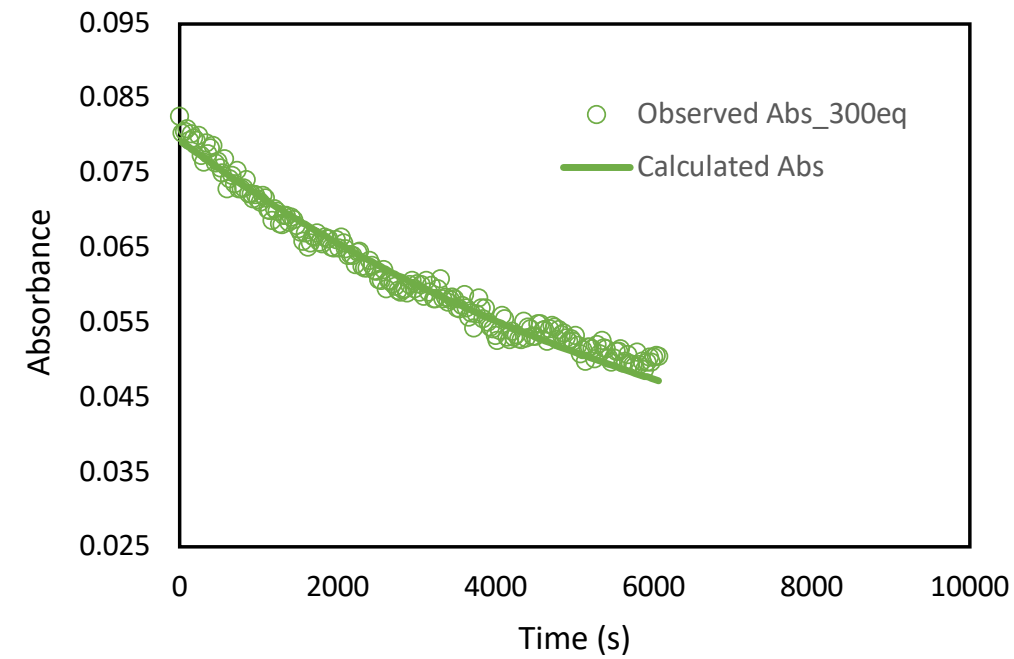

Figure S41. Kinetic run plot for a) 100 eq, b) 200 eq, c) 300 eq of Acetonitrile at $85^{\circ} \mathrm{C}$. 
a)

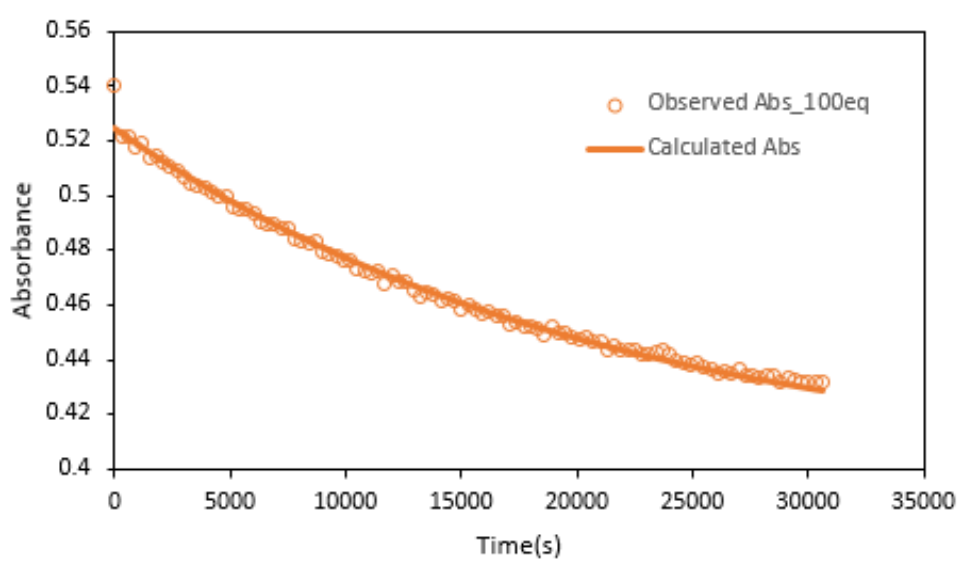

b)

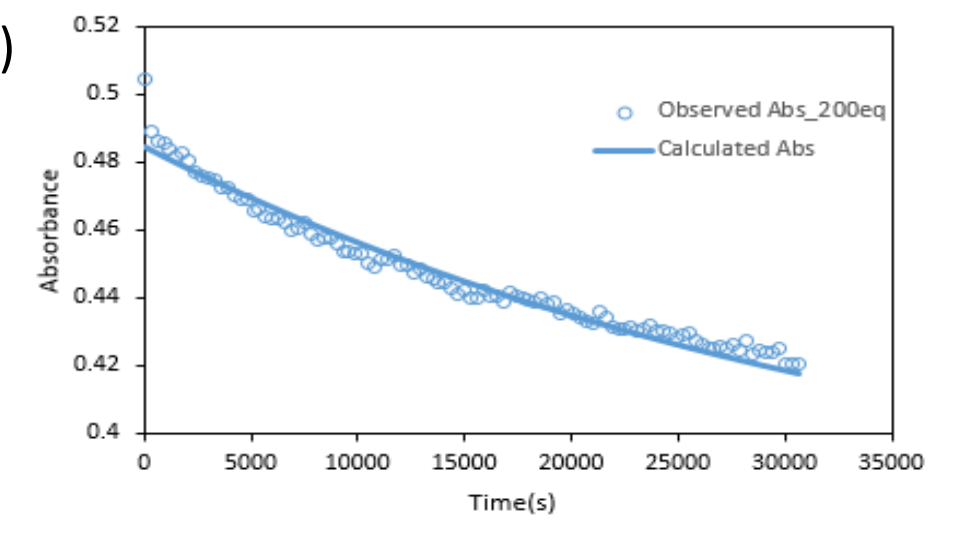

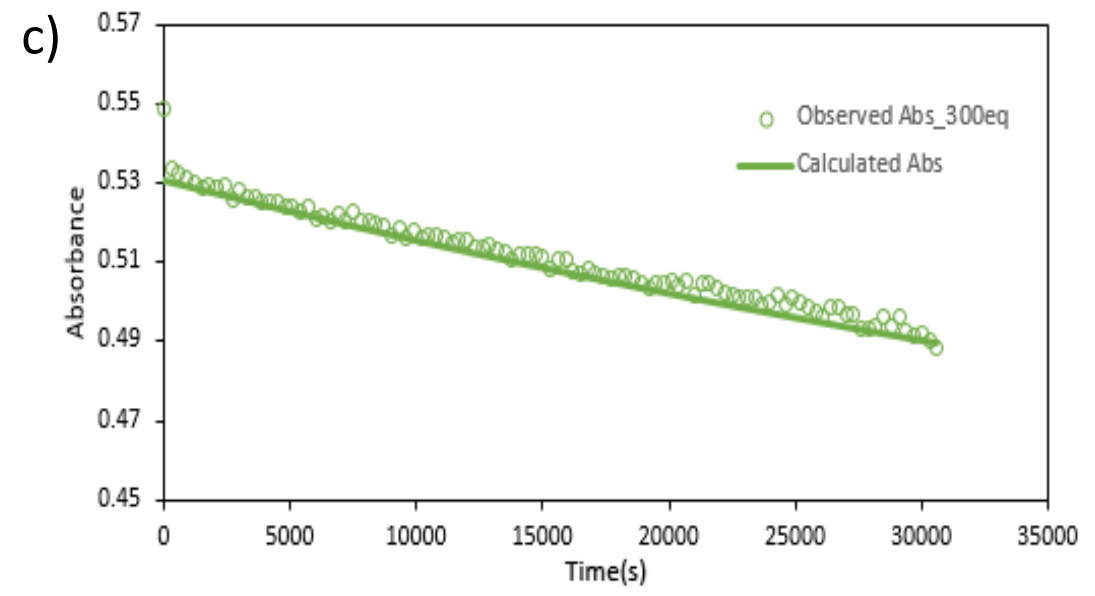

Figure S42. Kinetic run plot for a) 100 eq, b) 200 eq, c) 300 eq of Pyridine at $65^{\circ} \mathrm{C}$. 

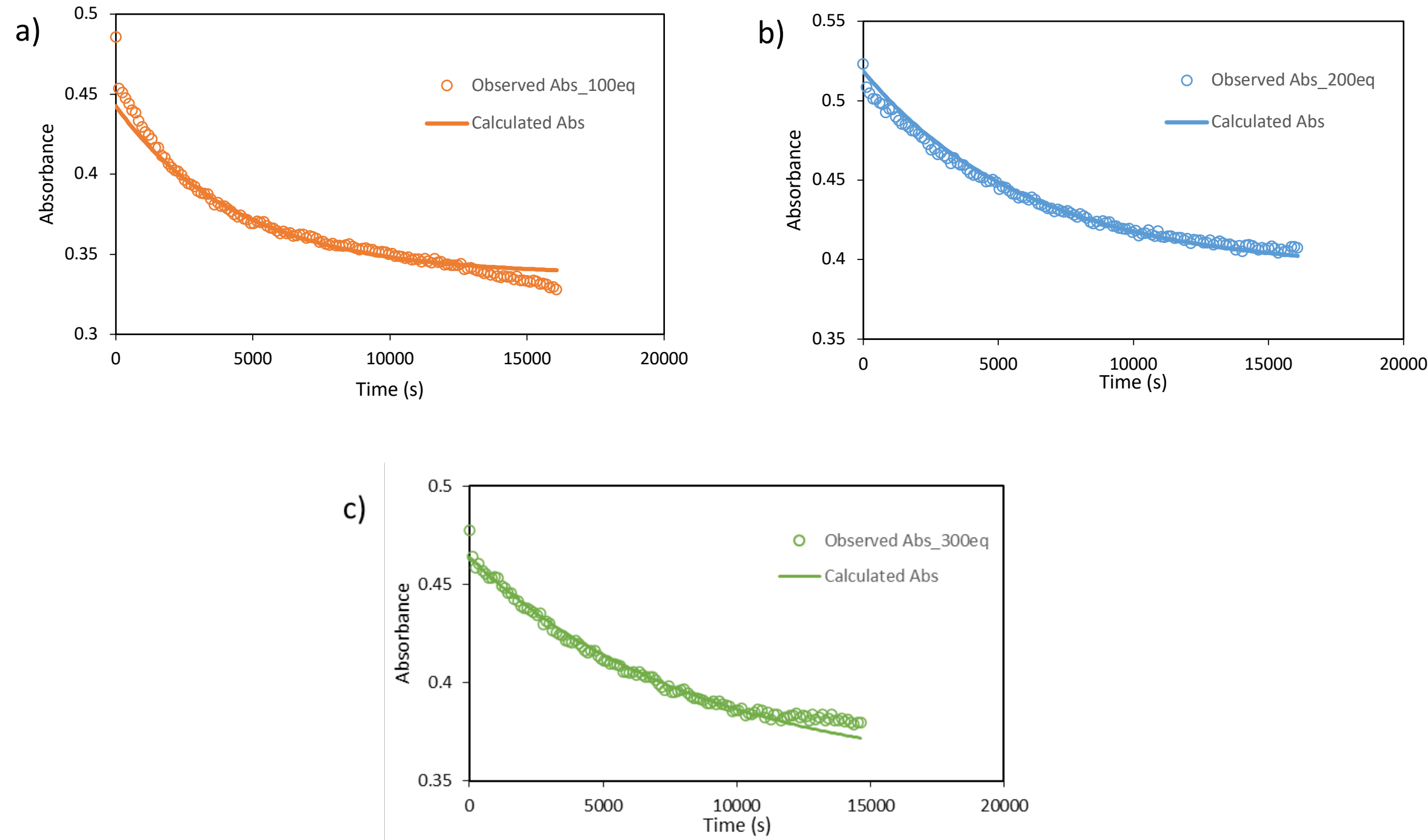

Figure S43. Kinetic run plot for a) 100 eq, b) 200 eq, c) 300 eq of Pyridine at $75^{\circ} \mathrm{C}$. 

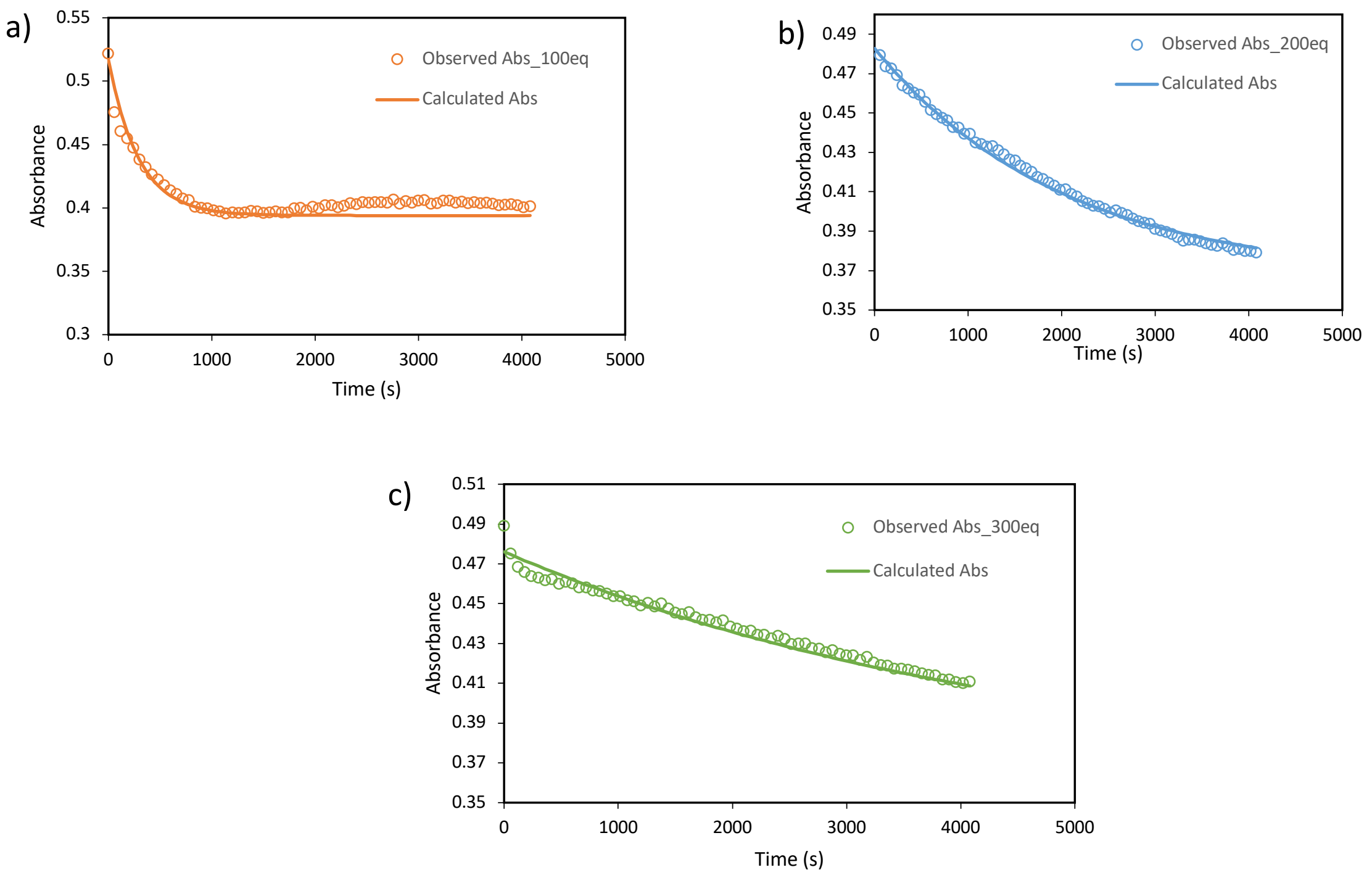

Figure S44. Kinetic run plot for a) 100 eq, b) 200 eq, c) 300 eq of Pyridine at $85{ }^{\circ} \mathrm{C}$. 

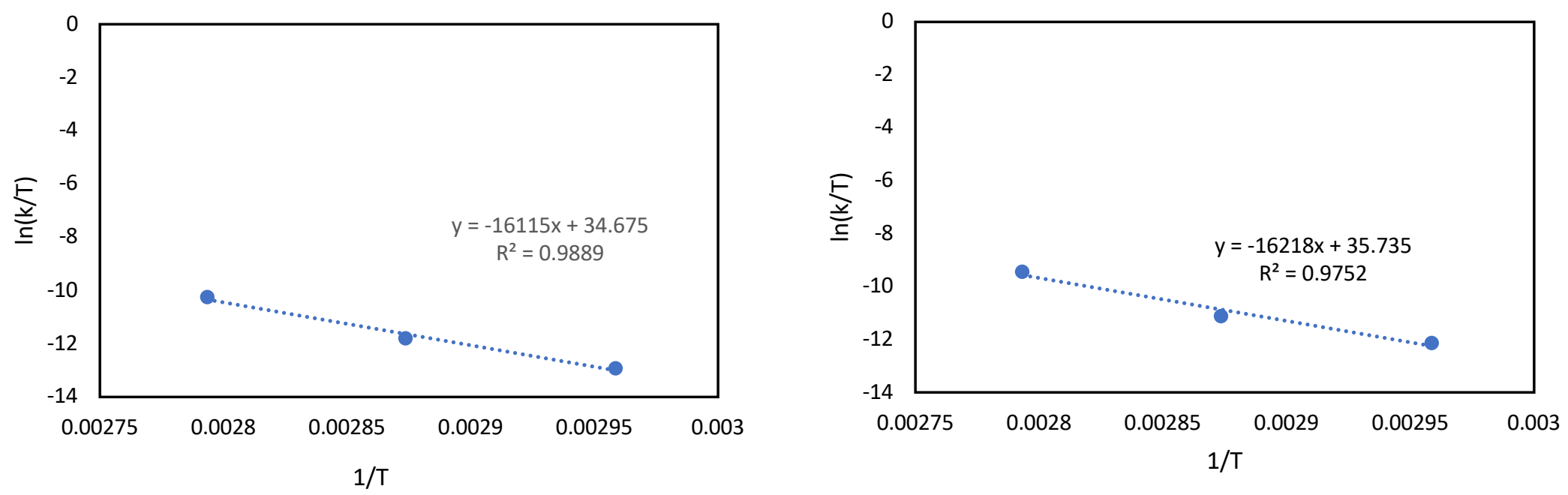

Figure S45. The Eyring plot for kinetic run with a) pyridine and b) acetonitrile. 


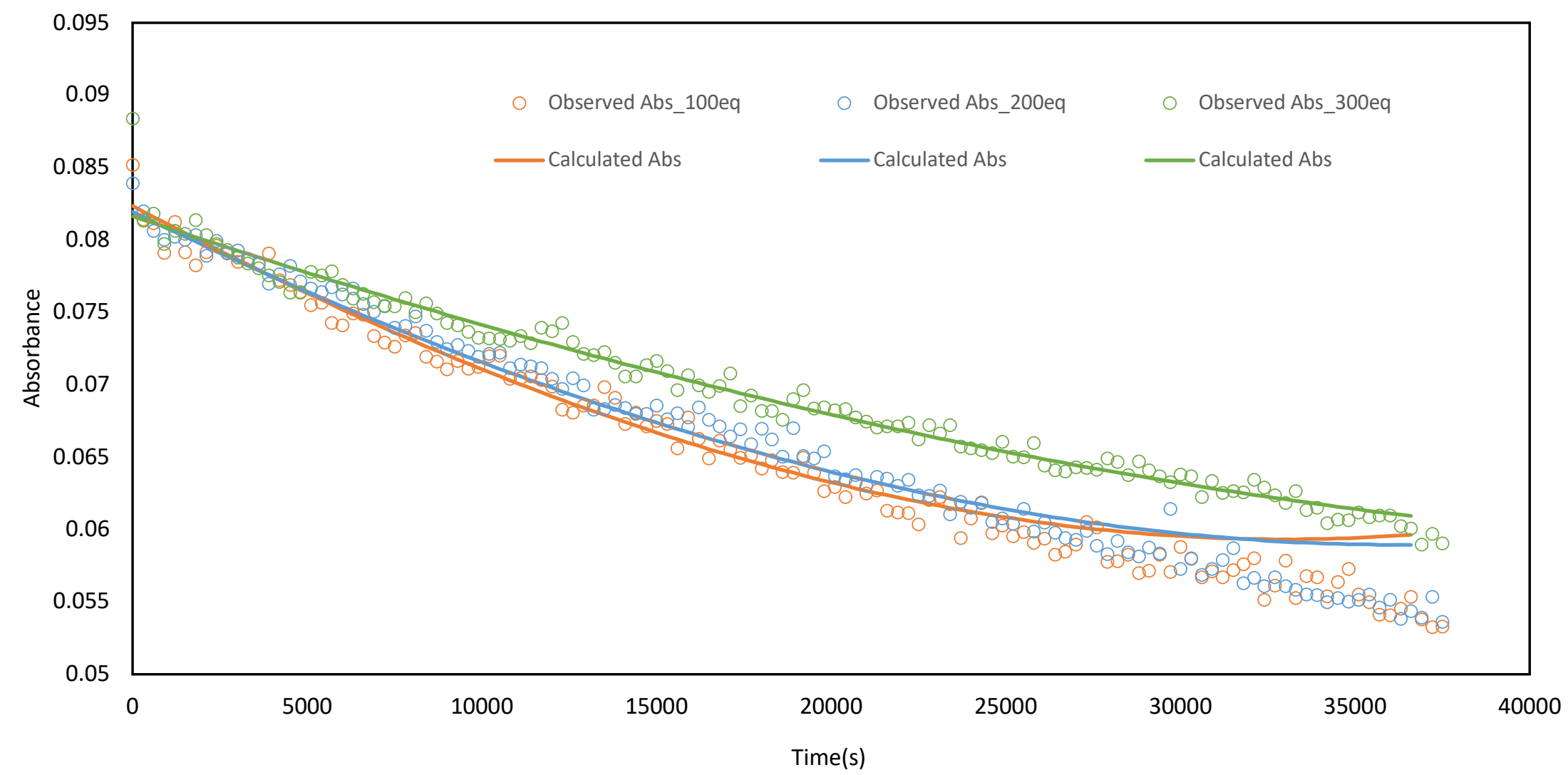

Figure S46. Kinetic run plots (according to model 2) of Acetonitrile at $75{ }^{\circ} \mathrm{C}$. 

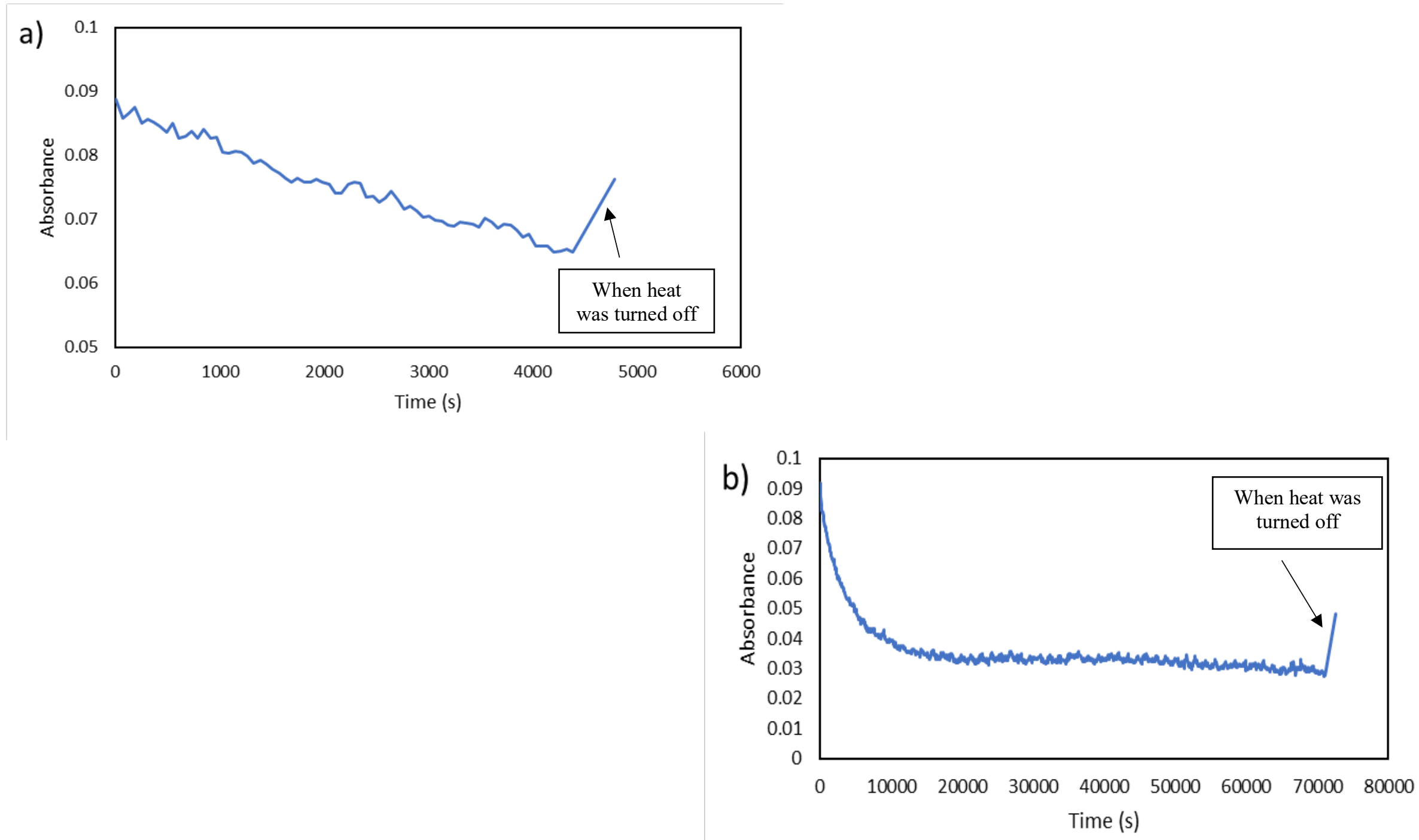

Figure S47. Plot of Absorbance Vs Time for $\left({ }^{\mathrm{tBu} P O C O P}\right) \operatorname{Ir}\left(\mathrm{PPh}_{3}\right)$ at a) $75{ }^{\circ} \mathrm{C}$, and b) $85{ }^{\circ} \mathrm{C}$. 